\title{
Algoritmos Eficientes para o Problema do \\ Orçamento Mínimo em Processos de Decisão Markovianos Sensíveis ao Risco
}

\author{
Daniel Augusto de Melo Moreira
}

\author{
DisSERTAÇÃO APRESENTADA \\ $\mathrm{AO}$ \\ Instituto De MatemáticA e EstatísticA \\ DA \\ Universidade DE SÃo PaUlo \\ PARA \\ OBTENÇÃO DO TÍTULO \\ DE \\ Mestre EM CiÊnCIAS \\ Programa: Ciência da Computação \\ Orientadora: Profa. Dra. Karina Valdivia Delgado
}

Durante o desenvolvimento deste trabalho o autor recebeu auxílio financeiro da CAPES

São Paulo, Janeiro de 2019 


\title{
Algoritmos Eficientes para o Problema do Orçamento Mínimo em Processos de Decisão Markovianos Sensíveis ao Risco
}

\author{
Esta versão da dissertação contém as correções e alterações sugeridas \\ pela Comissão Julgadora durante a defesa da versão original do trabalho, \\ realizada em 06/11/2018. Uma cópia da versão original está disponível no \\ Instituto de Matemática e Estatística da Universidade de São Paulo.
}

Comissão Julgadora:

- Prof ${ }^{a}$. Dr$^{\mathrm{a}}$. Karina Valdivia Delgado (orientadora) - EACH-USP

- Prof. Dr. Valdinei Freire da Silva - EACH-USP

- Prof. Dr. Graçaliz Pereira Dimuro - FURG 


\section{Resumo}

Moreira, D. A. M. Algoritmos Eficientes para o Problema do Orçamento Mínimo em Processos de Decisão Markovianos Sensíveis ao Risco. 2019. 70 p. (Dissertação de Mestrado) Instituto de Matemática e Estatística, Universidade de São Paulo, São Paulo, 2019.

O principal critério de otimização utilizado em Processos de Decisão Markovianos (MDPs) é minimizar o custo acumulado esperado. Embora esse critério de otimização seja útil, em algumas aplicações, o custo gerado por algumas execuções pode exceder um limite aceitável. Para lidar com esse problema foram propostos os Processos de Decisão Markovianos Sensíveis ao Risco (RS-MDPs) cujo critério de otimização é maximizar a probabilidade do custo acumulado não ser maior que um orçamento limite definido pelo usuário, portanto garantindo que execuções custosas de um MDP ocorram com menos probabilidade. Algoritmos para RS-MDPs possuem problemas de escalabilidade quando lidam com intervalos de custo amplos, uma vez que operam no espaço aumentado que enumera todos os possíveis orçamentos restantes. Neste trabalho é proposto um novo problema que é encontrar o orçamento mínimo para o qual a probabilidade de que o custo acumulado não exceda esse orçamento converge para um máximo. Para resolver esse problema são propostas duas abordagens: (i) uma melhoria no algoritmo TVI-DP (uma solução previamente proposta para RSMDPs) e (ii) o primeiro algoritmo de programação dinâmica simbólica para RS-MDPs que explora as independências condicionais da função de transição no espaço de estados aumentado. Os algoritmos propostos eliminam estados inválidos e adicionam uma nova condição de parada. Resultados empíricos mostram que o algoritmo RS-SPUDD é capaz de resolver problemas até $10^{3}$ vezes maior que o algoritmo TVI-DP e é até 26.2 vezes mais rápido que TVI-DP (nas instâncias que o algoritmo TVI-DP conseguiu resolver). De fato, é mostrado que o algoritmo RS-SPUDD é o único que consegue resolver instâncias grandes dos domínios analisados. Outro grande desafio em RS-MDPs é lidar com custos contínuos. Para resolver esse problema são definidos os RS-MDPs híbridos que incluem variáveis contínuas e discretas, além do orçamento limite definido pelo usuário. É mostrado que o algoritmo de programação dinâmica simbólica (SDP), existente na literatura, pode ser usado para resolver esse tipo de MDPs. Esse algoritmo foi empiricamente testado de duas maneiras diferentes: (i) comparado com os demais algoritmos propostos em um domínio em que todos são capazes de resolver e (ii) testado em um domínio que somente ele é capaz de resolver. Os resultados mostram que o algoritmo SDP para RS-MDP híbridos é capaz de resolver domínios com custos contínuos sem a necessidade de enumeração de estados, porém em troca do aumento do custo computacional.

Palavras-chave: Planejamento Probabilístico, Sensibilidade ao Risco, Processos de Decisão Markovianos. 


\section{Abstract}

Moreira, D. A. M. Efficient Algorithms for the Minimum Budget Problem in RiskSensitive Markov Decision Processe. 2019. 70 p. (Master's Thesis) - Instituto de Matemática e Estatística, Universidade de São Paulo, São Paulo, 2019.

The main optimization criterion used in Markovian Decision Processes (MDPs) is to minimize the expected cumulative cost. Although this optimization criterion is useful, in some applications the cost generated by some executions may exceed an acceptable threshold. In order to deal with this problem, the Risk-Sensitive Markov Decision Processes (RS-MDPs) were proposed whose optimization criterion is to maximize the probability of the cumulative cost not to be greater than an user-defined budget, thus guaranteeing that costly executions of an MDP occur with least probability. Algorithms for RS-MDPs face scalability issues when handling large cost intervals, since they operate in an augmented state space which enumerates the possible remaining budgets. In this work, we propose a new challenging problem of finding the minimum budget for which the probability that the cumulative cost does not exceed this budget converges to a maximum. To solve this problem, we propose: (i) an improved version of TVI-DP (a previous solution for RS-MDPs) and (ii) the first symbolic dynamic programming algorithm for RS-MDPs that explores conditional independence of the transition function in the augmented state space. The proposed algorithms prune invalid states and perform early termination. Empirical results show that RS-SPUDD is able to solve problems up to $10^{3}$ times larger than TVI-DP and is up to 26.2 times faster than TVI-DP (in the instances TVI-DP was able to solve). In fact, we show that RS-SPUDD is the only one that can solve large instances of the analyzed domains. Another challenging problem for RS-MDPs is handle continous costs. To solve this problem, we define Hybrid RS-MDPs which include continous and discrete variables, and the user-defined budget. In this work, we show that Symbolic Dynamic Programming (SDP) algorithm can be used to solve this kind of MDPs. We empirically evaluated the SDP algorithm: (i) in a domain that can be solved with the previously proposed algorithms and (ii) in a domain that only SDP can solve. Results shown that SDP algorithm for Hybrid RS-MDPs is capable of solving domains with continous costs, but with a higher computational cost.

Keywords: Probabilistic Planning, Risk-Sensitive, Markov Decision Process. 


\section{Sumário}

Lista de Abreviaturas vii

$\begin{array}{ll}\text { Lista de Símbolos } & \text { ix }\end{array}$

Lista de Figuras $\quad$ xi

Lista de Tabelas $\quad$ xiii

1 Introdução $\quad 1$

1.1 Motivação . . . . . . . . . . . . . . . . . . . . . . . . . . . . 2

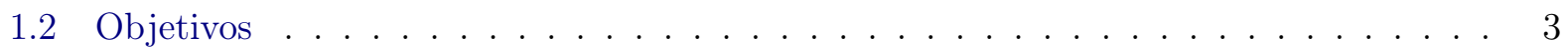

1.3 Organização . . . . . . . . . . . . . . . . . . . . . . 3

2 Representação de Funções e Operações $\quad 5$

2.1 Diagrama de Decisão Algébrica . . . . . . . . . . . . . . . . . . . . 5

2.2 Funções Baseadas em Casos . . . . . . . . . . . . . . . . . . . . 7

2.2.1 Definição de Funções Baseadas em Caso . . . . . . . . . . . . . . . . . . . 7

2.2 .2 Operações . . . . . . . . . . . . . . . . . . . . . 8

2.3 Diagrama de Decisão Algébrico Estendido . . . . . . . . . . . . . . . . . . . 10

3 Processos de Decisão Markovianos $\quad 15$

3.1 Definição Formal . . . . . . . . . . . . . . . . . . . . . 15

3.2 Algoritmos . . . . . . . . . . . . . . . . . . . . . . 16

3.2 .1 Iteração de Valor . . . . . . . . . . . . . . . . . . . . . 17

3.3 Iteração de Valor Topológico . . . . . . . . . . . . . . . . . . . . . . . . . . . 18

4 Processos de Decisão Markovianos Fatorados $\quad 21$

4.1 Definição Formal . . . . . . . . . . . . . . . . . . . . . . . . . . 21

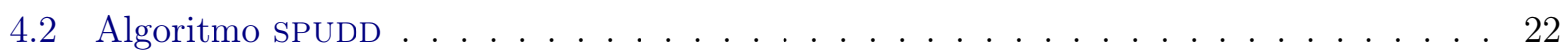

5 Processos de Decisão Markovianos Sensíveis ao Risco Enumerativos 25

5.1 Critérios de Sensibilidade ao Risco . . . . . . . . . . . . . . . . . 25

5.2 Definição Formal . . . . . . . . . . . . . . . . . . . . 25

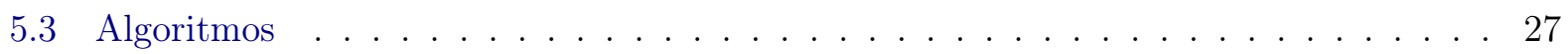


6 Processos de Decisão Markovianos Híbridos 35

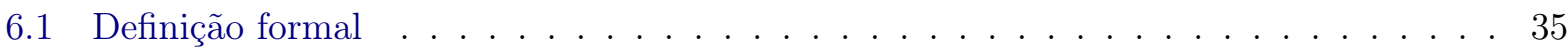

6.2 Algoritmo de Programação Dinâmica Simbólica . . . . . . . . . . . . . . . . . 37

7 Problema do Orçamento Mínimo $\quad 41$

7.1 Definição Formal . . . . . . . . . . . . . . . . . . . . . . . . . . 41

7.2 Iteração de Valor Topológico Melhorado (ITVI-DP) . . . . . . . . . . . . . . . . . . 43

7.3 Espaço de Estados Aumentados . . . . . . . . . . . . . . . . . . . . . . 45

8 Processos de Decisão Markovianos Sensíveis ao Risco Fatorados 47

8.1 Definição Formal . . . . . . . . . . . . . . . . . . . . . . . . 47

8.2 Algoritmo RS-SPUDD . . . . . . . . . . . . . . . . . . . . 47

9 Processos de Decisão Markovianos Híbridos Sensíveis ao Risco 53

9.1 Definição Formal . . . . . . . . . . . . . . . . . . . . . . . . . . 53

9.2 Resolução de HMdP Sensíveis ao Risco utilizando HMdPs . . . . . . . . . . . . . . . 53

10 Resultados $\quad 57$

10.1 Avaliação dos Algoritmos para RS-MDPs . . . . . . . . . . . . . . . 57

10.1.1 Domínio do Robô Navegador . . . . . . . . . . . . . . . . . . . . . . 57

10.1.2 Domínio SysAdmin . . . . . . . . . . . . . . . . . . . 58

10.1.3 Análise do Tempo de Convergência . . . . . . . . . . . . . . . . . . . . . 60

10.1.4 Análise do Consumo de Memória . . . . . . . . . . . . . . . . . . . . . . 61

10.2 Avaliação do Algoritmo para RS-MDPs Híbridos . . . . . . . . . . . . . . . . . . 62

10.2.1 Domínio do Robô Navegador ． . . . . . . . . . . . . . . . . . 63

10.2.2 Domínio Mars Rover . . . . . . . . . . . . . . . . . . . . . . . . . 64

10.2.3 Análise do Tempo de Convergência . . . . . . . . . . . . . . . . 65

11 Considerações Finais e Trabalhos Futuros $\quad 67$

$\begin{array}{ll}\text { Referências Bibliográficas } & 69\end{array}$ 


\title{
Lista de Abreviaturas
}

\author{
ADD Diagrama de Decisão Algébrico (Algebraic Decision Diagram) \\ BDD Diagrama de Decisão Binário (Binary Decision Diagram) \\ DAG $\quad$ Grafo Direcionado Acíclico (Directed Aciclic Graph) \\ DBN Redes Bayesianas Dinâmicas (Dynamic Bayesian Networks) \\ GD-MDP Processo de Decisão Markoviano direcionado a Meta (Goal-Directed Markov Decision Pro- \\ cess) \\ HMDP Processo de Decisão Markoviano Híbrido (Hybrid Markov Decision Process) \\ IPPC Competição Internacional de Planejamento Probabilístico (International Probabilistic \\ Planning Competition) \\ ITVI-DP Iteração de Valor Topológico Melhorado - Programação Dinâmica (Improved Topological \\ Value Iteration - Dynamic Programming) \\ MDP Processo de Decisão Markoviano (Markov Decision Process) \\ MRSVI RSVI Modificado (Modified RSVI) \\ RS-MDP Processo de Decisão Markoviano Sensiveis ao Risco (Risk-Sensitive Markov Decision Pro- \\ cess) \\ RSPUDD Planejamento Estocástico utilizando Diagramas de Decisão para RS-MDP (Risk Stochastic \\ Planning using Decision Diagrams) \\ RSVI Iteração de Valor para RS-MDP (Value Iteration for RS-MDP) \\ SCC Componente Fortemente Conexa (Strongly Connected Components) \\ SDP Programação Simbólica Dinâmica (Symbolic Dynamic Programming) \\ SPUDD Planejamento Estocástico utilizando Diagramas de Decisão (Stochastic Planning using De- \\ cision Diagrams) \\ SSP-MDP Problema do Caminho Estocástico Mais Curto (Stochastic Shortest Problem Markov deci- \\ sion Process) \\ TVI Iteração de Valor Topológico (Topological Value Iteration) \\ TVI-DFS Iteração de Valor Topológico - Busca em Profundidade (Topological Value Iteration - Depth- \\ First Search) \\ TVI-DP Iteração de Valor Topológico - Programação Dinâmica (Topological Value Iteration - Dy- \\ namic Programming) \\ VI Iteração de Valor (Value Iteration) \\ XADD Diagrama de Decisão Algébrico Estendido (Extended Algebraic Decision Diagram)
}




\title{
Lista de Símbolos
}

\author{
A Conjunto de ações \\ c Função de custo para o modelo fatorado \\ $c_{\max }$ Custo máximo de um RS-MDP \\ $C$ Função de custo \\ $C_{f} \quad$ Função de custo fatorada \\ $D_{b} \quad$ Conjunto de DBNs \\ E Arestas do grafo de SCCs \\ $f^{\pi} \quad$ Função utilidade seguindo a política $\pi$ \\ $f^{*} \quad$ Função utilidade ótima \\ $f^{i} \quad$ Função utilidade na $i$-ésima iteração \\ $\vec{g} \quad$ Vetor de atribuição de $G$ \\ $G \quad$ Conjunto de vetores de estado meta \\ $h \quad$ Histórico de execução \\ $M \quad$ Um MDP \\ $M_{f} \quad$ Um MDP fatorado \\ $p \quad$ Probabilidade de custo-limite fatorada \\ $p^{*} \quad$ Probabilidade de custo-limite ótima fatorada \\ $p^{i} \quad$ Probabilidade de custo-limite fatorada na $i$-ésima iteração \\ pa Conjunto de pais de uma variável \\ $P \quad$ Probabilidade de custo-limite \\ $P^{*} \quad$ Probabilidade de custo-limite ótima \\ $P^{i} \quad$ Probabilidade de custo-limite na $i$-ésima iteração \\ $\operatorname{Pr} \quad$ Distribuição de probabilidade condicional \\ $Q_{a}^{i} \quad$ Valor da ação $a$ em uma dada iteração $i$ \\ $R \quad$ Função de recompensa \\ $s_{0} \quad$ Estado inicial \\ $S \quad$ Conjunto de estados \\ $\mathbb{S}_{a} \quad$ Conjunto de estados aumentados \\ $S_{g} \quad$ Conjunto de estados Meta \\ $T$ Função de transição \\ $\vec{x} \quad$ Vetor de atribuição de $\vec{X}$ \\ $\vec{X} \quad$ Vetor de variáveis de estado \\ $Y \quad$ Conjunto de componentes fortemente conexas
}


$\delta \quad$ Erro máximo entre duas funções utilidade subsequentes

$\epsilon \quad$ Erro máximo

$\Gamma \quad$ Variável do orçamento limite

$\pi \quad$ Política de um MDP

$\pi^{*} \quad$ Política ótima de um MDP

$\theta_{u} \quad$ Orçamento (limite de gasto) inicial

$\theta_{\text {min }}$ Orçamento mínimo para o Problema do Orçamento Mínimo

$\Theta \quad$ Conjunto de possíveis orçamentos 


\section{Lista de Figuras}

1.1 Exemplo do domínio do Robô Navegador. O robô se locomove em uma grade e tem como objetivo chegar a célula marcada por $G$. Movimentos realizados em células mais escuras possuem maior probabilidade de fazer o robô desaparecer, enquanto movimentos em células brancas tem probabilidade zero de fazer o robô desaparecer. . 1

2.1 Probabilidade condicional apresentada na Tabela 2.1 representada por um ADD ordenado (fig. esquerda) e um ADD ordenado e reduzido (fig. direita). As linhas pontilhadas representam a valoração falsa (0) e as linhas sólidas a valoração verdadeira

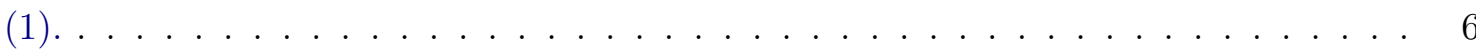

2.2 Funções $f$ e $g$ da Tabela 2.2 representadas por ADDs (parte superior) e resultado das operações de (a) soma, (b) minimização e (c) marginalização sobre a variável $X_{2}$. . .

2.3 Representação da função baseada em casos $f$ da Equação 2.14 por um XADD, em que linhas pontilhadas representam a valoração falsa (0) e as linhas sólidas a valoração verdadeira (1). . . . . . . . . . . . . . . . . . . . . . 11

2.4 Exemplo da operação de substituição para a função $f$ (Equação 2.14) $\operatorname{com} \delta=\left\{y_{3}=\right.$

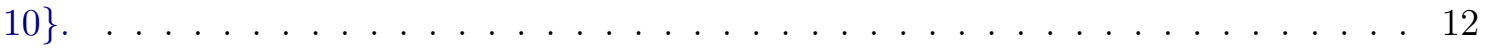

2.5 Exemplo de um XADD $G$ desordenado. . . . . . . . . . . . . . . . . . 13

2.6 Exemplo das operações realizadas respectivamente na linha 5, 6 e 7 do Algoritmo 1 para ordenar o XADD $G \ldots \ldots \ldots \ldots \ldots \ldots$

2.7 Exemplo da operação de maximização para as funções $f_{\left\{y_{3}=10\right\}}$ e $f_{2} \ldots \ldots \ldots$

3.1 Exemplo de um MDP. Transições são denotadas por setas, ações são denotadas por quadrados, estados são denotados por círculos e o custo de cada par estado-ação é mostrado no canto superior-esquerdo. . . . . . . . . . . . . . . . . . . . . 17

3.2 SCCs (retângulos arredondados) para o exemplo de MDP da Figura $3.1 \ldots \ldots$

5.1 Exemplo do espaço de estados aumentado para $\theta_{u}=2$ referente ao MDP da Figura 3.1, em que transições são denotadas por arcos e estados aumentados por círculos. 27

5.2 Execução do algoritmo TVI-DFS para o exemplo da Figura 5.1, em que SCCs são denotadas por retângulos arredondados. . . . . . . . . . . . . . . . 30

5.3 Execução do algoritmo TVI-DP para o exemplo da Figura 5.1, em que SCCs são denotadas por retângulos arredondados. 
7.1 Exemplo usado na prova do Teorema 4. A figura mostra três possibilidades de históricos: (a) um histórico $h(\pi)$ com custo acumulado menor ou igual que $\theta$, em que $\pi$ é a política que maximiza a probabilidade de custo-limite $P^{\pi}(s, \theta)$; (b) o mesmo histórico $h(\pi)$ mostrado em (a), porém com custo acumulado menor ou igual que $\theta+1$, em que $\pi$ é a política que maximiza a probabilidade de custo-limite $P^{\pi}(s, \theta+1)$; e (c) um novo histórico com custo acumulado igual a $\theta+1$, em que $\pi$ é a política que maximiza a probabilidade de custo-limite $P^{\pi}(s, \theta+1)$.

7.2 Grafo das SCCs para os estados aumentados gerado pelo algoritmo ITVI-DP para o RS-MDP da Figura 5.1. . . . . . . . . . . . . . . . . . . . 45

8.1 Exemplo do cálculo de $W_{D D}^{0}\left(\cdot, a_{1}, 25, \cdot\right) \ldots \ldots \ldots \ldots \ldots \ldots$

8.2 Exemplo da marginalização sobre a variável $x^{\prime} \ldots \ldots \ldots \ldots$. . . . . . . . . 50

10.1 Exemplo simples do robô navegador: (a) grade com coordenadas $X$ e $Y$; (b) DBN para a ação ir-para-cima em que as coordenadas são expressas em binário; (c) Representação em ADD da CPT para a variável $X_{1}^{\prime}$; (d) Representação em ADD do custo imediato com $\alpha=10 \ldots \ldots \ldots \ldots \ldots \ldots \ldots$

10.2 Exemplo simples do SysAdmin: (a) Rede de computadores interconectados; (b) DBN para a ação de reiniciar computador 1; (c) Representação em ADD da CPT para as variáveis $C_{1}$ e $C_{2}$; e (d) Representação em ADD do custo imediato em que level $\left(c_{j}\right)=$

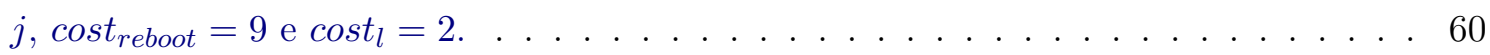

10.3 Tempo de convergência em $\log _{10}$ de milissegundos para o domínio do Robô Navegador. 60

10.4 Tempo de convergência em $\log _{10}$ de milissegundos para o domínio SysAdmin. . . . . 61

10.5 Tempo de convergência para o domínio do Robô Navegador com $\theta_{u}$ variando entre

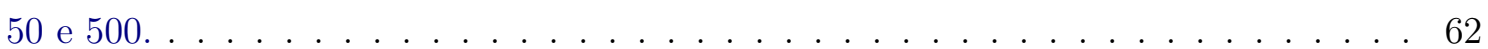

10.6 Tempo de convergência para o domínio do SysAdmin com $\theta_{u}$ variando entre 50 e $500 . .62$

10.7 Consumo de memória em Kbytes para o domínio do Robô Navegador. . . . . . . . . 63

10.8 Consumo de memória em Kbytes para o domínio SysAdmin. . . . . . . . . . . . . . 63

10.9 Tempo de convergência para o domínio do Robô Navegador. . . . . . . . . . . . . . 65

10.10Tempo de convergência para a primeira configuração do domínio Mars Rover. . . . . 66

10.11Tempo de convergência para a segunda configuração do domínio Mars Rover. . . . . 66 


\section{Lista de Tabelas}

2.1 Exemplo de uma tabela de probabilidade condicional . . . . . . . . . . . . . . . 5

2.2 Resultado da aplicação de algumas operações para duas funções $f$ e $g$. . . . . . 6

4.1 Exemplo de uma tabela de probabilidade condicional $(\mathrm{CPT}) \ldots \ldots$. . . . . . . . 22

5.1 Probabilidades de custo-limite calculadas pelo algoritmo TVI-DFS para cada estado aumentado $\left(s_{i}, \theta\right)$ considerando $\theta_{u}=2 \ldots \ldots \ldots \ldots \ldots \ldots$

5.2 Probabilidades de custo-limite calculadas pelo algoritmo TVI-DP para cada estado

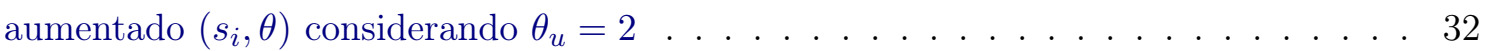

7.1 Probabilidades de custo-limite calculadas pelo algoritmo TVI-DP para cada estado aumentado $\left(s_{i}, \theta\right)$ considerando $\theta_{u}=8 \ldots \ldots \ldots \ldots \ldots \ldots$ 


\section{Capítulo 1}

\section{Introdução}

Um Processo de Decisão Markoviano (MDP) é um modelo matemático comumente utilizado em planejamento probabilístico em que o objetivo é encontrar uma política ótima (mapeamento de estados em ações) que minimize o custo acumulado esperado. Alguns dos algoritmos propostos para solucionar MDPs são os algoritmos de Iteração de Valor (Value Iteration - VI) [Put94] e Iteração de Valor Topológico (Topological Value Iteration - TVI) [DG07].

Embora o critério de otimização que minimiza o custo acumulado esperado seja útil para uma variedade de aplicações, pois garante que no decorrer de muitas execuções o custo acumulado seja próximo ao estimado; esse pode ser um critério inaceitável para outras aplicações em que o número de execuções é limitado. Neste caso, as políticas encontradas não garantem uma variância baixa e, portanto, podem gerar execuções com custo exorbitante. Por exemplo, quando uma política somente é necessária em ocasiões esporádicas que requerem alta probabilidade de sucesso (e.g. voo espacial não-tripulado ou operações militares) a otimização do custo acumulado esperado não é uma boa solução.

Para resolver esse problema foi proposto o Processo de Decisão Markovianos Sensível ao Risco (RS-MDP) [YLY98, HYV14] em que o critério de otimização é baseado na maximização da probabilidade do custo acumulado não ser maior que um dado limite, chamada de probabilidade de custo-limite, e que inclui um conjunto de estados meta e um custo limite definido pelo usuário. A seguir é descrito o problema do Robô Navegador que será usado para introduzir a intuição sobre risco.

Exemplo 1. Robô Navegador: Um robô deve navegar de uma posição inicial até uma posição meta em uma grade de tamanho $n \times m$. O robô pode se mover para qualquer posição adjacente da sua posição atual, porém existe uma probabilidade do robô quebrar que aumenta conforme ele se aproxima da coluna em que está a meta (Figura 1.1).

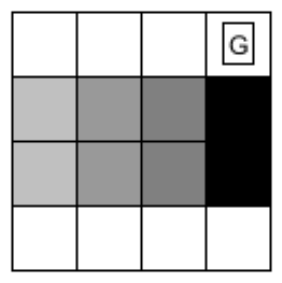

Figura 1.1: Exemplo do domínio do Robô Navegador. O robô se locomove em uma grade e tem como objetivo chegar a célula marcada por $G$. Movimentos realizados em células mais escuras possuem maior probabilidade de fazer o robô desaparecer, enquanto movimentos em células brancas tem probabilidade zero de fazer o robô desaparecer.

No exemplo 1, o robô toma caminhos mais arriscados (maior probabilidade de quebrar) quando o custo limite é baixo e escolhe caminhos menos arriscados (menor probabilidade de quebrar) quando 
o custo limite é alto. Assim, nesse exemplo, o custo limite está relacionado com a quantidade máxima de movimentos permitido ao robô.

Hou et al (2014) propôs modificações eficientes do algoritmo clássico de Iteração de Valor Topológico [DG07] para resolver RS-MDPs, chamados de TVI-DFS e TVI-DP. Em particular, o algoritmo TVI-DP encontra políticas ótimas para diferentes custos limite $\theta_{u}$.

Neste trabalho é provado que o algoritmo TVI-DP pode ser modificado para encontrar o orçamento mínimo $\theta_{\min }$ para o qual a probabilidade do custo acumulado converge para um valor máximo (dado um custo limite $\theta_{u}$ grande o suficiente). Tal problema é chamado de Problema do Custo Mínimo. No exemplo do robô navegador, $\theta_{\text {min }}$ é o orçamento mínimo (que está relacionado com a quantidade de movimentos mínima) que garante que o robô atinga a posição meta com máxima probabilidade.

Além disso, é mostrado que o algoritmo modificado proposto, chamado de TVI-DP melhorado (Improved TVI-DP - ITVI-DP), retorna o valor $\theta_{\text {min }}$ além de também solucionar o problema original com maior eficiência em termos de tempo de execução e memória requerida do que o algoritmo TVI-DP original.

Embora, os algoritmos TVI-DFS, TVI-DP e o algoritmo proposto ITVI-DP serem eficientes, eles requerem uma representação enumerativa do RS-MDP o que pode levar desnecessariamente a um raciocínio complexo que poderia ser simplificado por uma representação fatorada (ou simbólica). Para tratar esse problema este trabalho propõe abordar RS-MDPs em sua representação fatorada, além de propor um algoritmo eficiente para soluciona-lo, chamado de RSSPUDD (Risked Sensitive Stochastic Planning with Decision Diagram). RSSPUDD é baseado na solução clássica de MDPs fatorados, o algoritmo SPUDD [HSAHB99]. Além disso, RSSPUDD assim como o ITVI-DP proposto também retorna $\theta_{\min }$.

Os algoritmos propostos (ITVI-DP e RSSPUDD) foram avaliados em instâncias de dois domínios do IPPC ${ }^{1}$ 2011: Robô Navegador (Navigation) e SysAdmin. Os resultados mostram que o algoritmo RSSPUDD possui melhor desempenho quando comparado com os demais algoritmos enumerativos baseados em TVI. Além disso, ITVI também demonstrou ser melhor quando comparado com TVI-DP.

Apesar desses bons resultados, os algoritmos propostos podem falhar ao solucionar problemas complexos, em especial problemas que possuem variáveis contínuas para representar os estados. Para tais problemas, é mostrado que pode ser utilizado o arcabouço de Processos de Decisão Markovianos Híbridos (HMDPs) [SM06]. Tal solução é analisada nos domínios do Robô Navegador e Mars Rover [SDdB11].

\subsection{Motivação}

Motivados pelo recente reaparecimento de Processos de Decisão Markovianos Sensíveis ao Risco na área de Inteligência Artificial, e o sucesso de algoritmos que utilizam representações fatoradas de MDP; este trabalho formaliza a versão fatorada para Processos de Decisão Markovianos Sensíveis ao Risco e propõe um algoritmo eficiente baseado em SPUDD.

Outra grande motivação foi encontrada no desejo de garantir que nenhum recurso alocado seja desnecessariamente alocado em algumas tarefas modeladas em RS-MDPs. Assim esse trabalho propõe um novo problema interessante no âmbito de RS-MDPs: Como encontrar o orçamento mínimo inicial de modo que, a partir desse orçamento, as probabilidades de custo-limite ótimas sejam máximas?. Este trabalho também demonstra que a resolução do problema do orçamento mínimo proposto pode conduzir a otimizações em algoritmos anteriormente propostos na literatura.

Por fim, motivados pela falta de algoritmos para resolver RS-MDPs com variáveis contínuas, neste trabalho demonstramos como utilizar o arcabouço de HMDPs para obter uma solução eficiente.

\footnotetext{
${ }^{1}$ Competição Internacional de Planejamento Probabilístico - Internacional Probabilistic Planning Competition
} 


\subsection{Objetivos}

O objetivo principal deste trabalho de mestrado é propor novas abordagens eficientes para a solução de RS-MDPs.

Os objetivos específicos presentes neste trabalho incluem:

- Desenvolver otimizações para algoritmos enumerativos encontrados na literatura para resolver RS-MDPs;

- Propor a definição formal fatorada de RS-MDP;

- Desenvolver um novo algoritmo baseado em SPUDD para RS-MDPs fatorados;

- Solucionar RS-MDPs com variáveis contínuas.

\subsection{Organização}

Este trabalho está organizado da seguinte forma: nos Capítulos 2, 3, 4, 5 e 6 são abordados respectivamente os conceitos de representação de funções, Processos de Decisão Markovianos (MDP), Processos de Decisão Markovianos Fatorados, Processos de Decisão Markovianos Sensíveis ao Risco (RS-MDP) e Processos de Decisão Markovianos Híbridos (HMDP), assim como alguns dos algoritmos propostos na literatura para resolvê-los. Nos Capítulos 7, 8 e 9 são apresentadas as contribuições deste trabalho de mestrado. No Capítulo 7 é apresentado o problema do orçamento mínimo e melhorias realizadas no algoritmo TVI-DP, para resolver RS-MDPs. No Capítulo 8 são apresentados a definição de Processos de Decisão Markovianos Sensíveis ao Risco Fatorados e o primeiro algoritmo para resolvê-lo. No Capítulo 9 é apresentada a abordagem baseada em Processos de Decisão Markovianos Híbridos para o tratamento de variáveis contínuas em RS-MDPs. No Capítulo 10 são analisadas empiricamente as soluções propostas em diferentes domínios. Finalmente, no Capítulo 11 as considerações finais e trabalhos futuros são abordados. 


\section{Capítulo 2}

\section{Representação de Funções e Operações}

Neste capítulo são descritos o diagrama de decisão algébrico, funções baseadas em casos e o diagrama de decisão algébrico estendido, assim como seus respectivos operadores. Tais representações são usadas para representar funções nos Processos de Decisão Markovianos e serão retomadas a partir do Capítulo 4.

\subsection{Diagrama de Decisão Algébrica}

Diagramas de Decisão Algébricas (Algebraic Decision Diagrams - ADDs) [BFG+93] são uma generalização de Diagramas de Decisão Binários (Binary Decision Diagrams - BDDs) [Bry86] que estendem a representação de funções binárias para funções com valores reais, i.e., um ADD expressa de forma compacta funções da forma $f:\{0,1\}^{n} \rightarrow \mathbb{R}$.

Definição 1. Um Diagrama de Decisão Algébrico (ADD) é uma representação de uma função de variáveis booleanas $f:\{0,1\}^{n} \rightarrow \mathbb{R}$ como um grafo acíclico dirigido $G=(V, A)$, tal que:

- $V=D \cup F$ em que $D$ é um conjunto de nós de decisão (ou nós internos), que inclui o nó raiz; e $F$ é um conjunto de nós folhas (ou nós terminais). Cada nó terminal $f \in F$ assume um valor real $(f \in \mathbb{R})$. Cada nó interno $d \in D$ representa uma variável binária $X_{i}$ e possuí dois nós filhos $v_{0}, v_{1} \in V$, que são a raiz do ADD esquerdo $v_{0_{\text {add }}}$ e direito $v_{1_{\text {add }}}$, respectivamente.

- Cada aresta $a \in A$ de um nó $d$ para um nó $v_{0}$ (ou $v_{1}$ ) representa uma atribuição de 0 (ou 1 ) para a variável $X_{i}$.

Um ADD é ordenado se as variáveis aparecem na mesma ordem em todos os caminhos entre o nó raiz e os nós terminais. Um exemplo de um ADD ordenado para representar a probabilidade condicional apresentada na Tabela 2.1 é ilustrado à esquerda da Figura 2.1.

Tabela 2.1: Exemplo de uma tabela de probabilidade condicional

\begin{tabular}{|c|c|c|c|}
\hline$X_{1}$ & $X_{2}$ & $X_{3}$ & $\operatorname{Pr}\left(X_{1}^{\prime}=1 \mid X_{1}, X_{2}, X_{3}\right)$ \\
\hline 0 & 0 & 0 & 0.30 \\
\hline 0 & 0 & 1 & 0.30 \\
\hline 0 & 1 & 0 & 0.95 \\
\hline 0 & 1 & 1 & 0.10 \\
\hline 1 & 0 & 0 & 0.95 \\
\hline 1 & 0 & 1 & 0.95 \\
\hline 1 & 1 & 0 & 0.20 \\
\hline 1 & 1 & 1 & 0.15 \\
\hline
\end{tabular}

Definição 2. Dois grafos $G=\left(V_{G}, A_{G}\right)$ e $H=\left(V_{H}, A_{H}\right)$ são considerados isomórficos se existe uma função bijetora $g: V_{G} \rightarrow V_{H}$ tal que: 
- Para quaisquer par de vértices $u$ e $v$ em $V_{G}$ existe uma aresta de $u$ para $v$ em $A_{G}$ se e somente se existe uma aresta de $g(u)$ para $g(v)$ em $A_{H}$; e

- $u$ e $v$ possuem respectivamente a mesma etiqueta (variável binária $X_{i}$ ou valor real) que $g(u)$ e $g(v)$ em $H$.

Um ADD que obedece as seguintes regras é considerado reduzido:

1. Todos os subgrafos isomórficos são unificados;

2. Todos os nós que possuem filhos isomórficos são removidos.

O ADD ilustrado à direita da Figura 2.1 mostra o resultado dessas compactações para o ADD ilustrado à esquerda. Será feita a suposição, daqui para frente, que todos os ADDs são ordenados e reduzidos. Essas propriedades são particularmente úteis na checagem de equivalência entre funções e outras operações entre ADDS como as apresentadas a seguir. Além disso, não faremos qualquer distinção entre um nó $v$ e sua respectiva etiqueta.

Dada uma função $f:\{0,1\}^{n} \rightarrow \mathbb{R}$ representada por um ADD e um caminho nesse ADD que começa no nó raiz e termina em um nó folha $t$, o caminho representa uma atribuição total (ou parcial) das variáveis booleanas para o qual a função $f$ retorna o valor $t$. Ao percorrer tal caminho até o nó folha $t$, a cada variável pertencente ao caminho é atribuída o respectivo valor 0 (ou 1) caso seu nó filho seja $v_{0}\left(\right.$ ou $v_{1}$ ). Por exemplo, seguindo o único caminho até o nó folha de valor 0.3 no ADD à direita da Figura 2.1, tem-se a seguinte valoração das variáveis booleanas $\left(X_{1}=0, X_{2}=0\right)$. Assim, esse caminho representa $\operatorname{Pr}\left(X_{1}^{\prime}=1 \mid X_{1}=0, X_{2}=0\right)=0.3$.
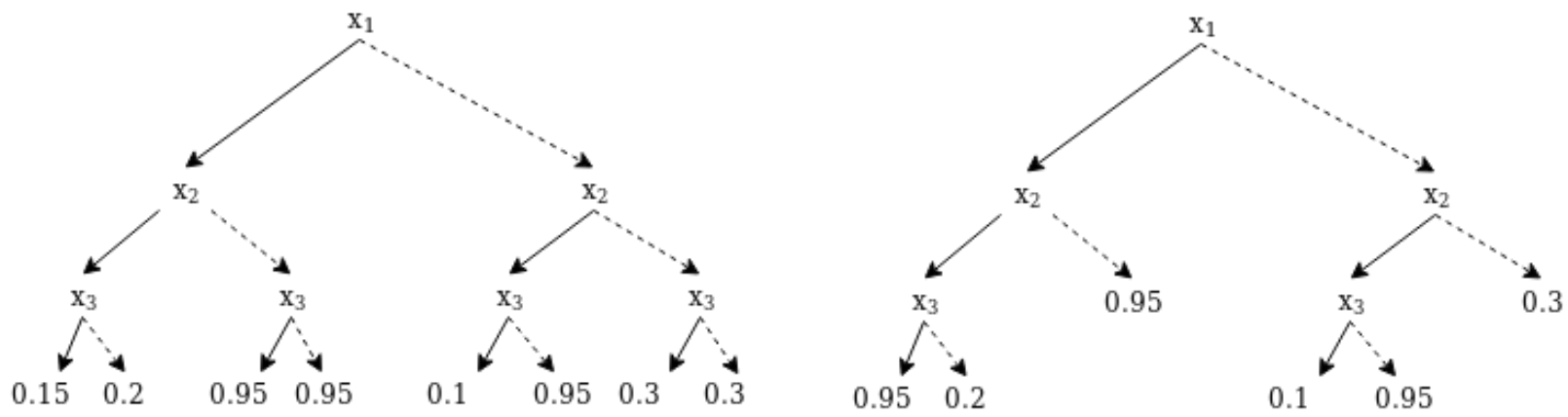

Figura 2.1: Probabilidade condicional apresentada na Tabela 2.1 representada por um ADD ordenado (fig. esquerda) e um ADD ordenado e reduzido (fig. direita). As linhas pontilhadas representam a valoração falsa (0) e as linhas sólidas a valoração verdadeira (1).

Além da representação compacta de funções, ADDs são capazes de efetuar eficientemente operações binárias como multiplicação $(\otimes)$, soma $(\oplus)$, subtração $(\ominus)$, minimização $(\min (f, g))$ e maximização $(\max (f, g))$; e operações unárias como a marginalização, também conhecida como sum-out $\left(\sum(f)\right)$.

Na Tabela 2.2 é mostrado um exemplo da aplicação de cada uma das operações citadas acima para duas funções $f$ e $g$. Além disso, ilustramos na Figura 2.2 as operações de soma, minimização e marginalização sobre a variável $X_{2}$ correspondentes a Tabela 2.2 .

Tabela 2.2: Resultado da aplicação de algumas operações para duas funções $f$ e $g$

\begin{tabular}{|c|c|c|c|c|c|c|c|c|c|c|}
\hline$x_{1}$ & $x_{2}$ & $f\left(x_{1}\right)$ & $g\left(x_{1}, x_{2}\right)$ & $f \oplus g$ & $f \ominus g$ & $f \otimes g$ & $\min (f, g)$ & $\max (f, g)$ & $\sum_{x_{1}}(f)$ & $\sum_{x_{2}}(g)$ \\
\hline 0 & 0 & 0.3 & 0.2 & 0.5 & 0.1 & 0.06 & 0.2 & 0.3 & 1.0 & 0.6 \\
\hline 0 & 1 & 0.3 & 0.4 & 0.7 & -0.1 & 0.12 & 0.3 & 0.4 & 1.0 & 0.6 \\
\hline 1 & 0 & 0.7 & 0.25 & 0.95 & 0.45 & 0.175 & 0.25 & 0.7 & 1.0 & 0.4 \\
\hline 1 & 1 & 0.7 & 0.15 & 0.85 & 0.55 & 0.105 & 0.15 & 0.7 & 1.0 & 0.4 \\
\hline
\end{tabular}



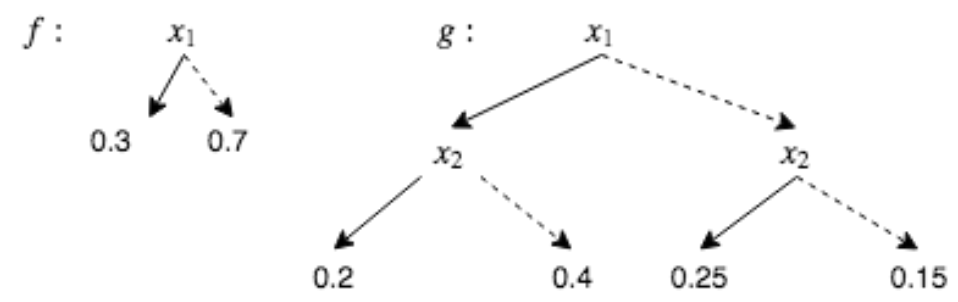

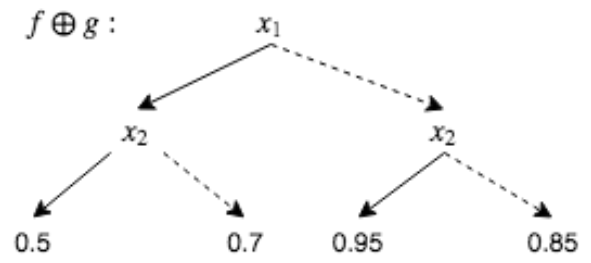

(a)

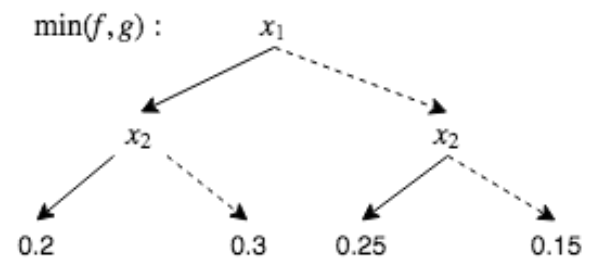

(b)

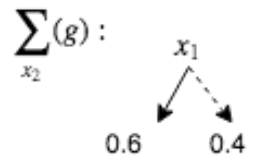

(c)

Figura 2.2: Funções $f$ e $g$ da Tabela 2.2 representadas por ADDs (parte superior) e resultado das operações de (a) soma, (b) minimização e (c) marginalização sobre a variável $X_{2}$.

ADDs podem ser utilizados para representar de maneira compacta as funções e realizar eficientemente operações para resolver Processos de Decisão Markovianos, como será visto no Capítulo 4.

\subsection{Funções Baseadas em Casos}

Uma função baseada em casos é uma função definida por múltiplas sub-funções, em que cada sub-função é aplicada em certo intervalo (definido por uma fórmula lógica) do domínio da função baseada em casos. Por exemplo, para uma função linear baseada em casos podem existir diversas funções lineares que definem o domínio da função baseada em casos. A seguir é definida formalmente uma função baseada em casos e são apresentadas as operações que podem ser realizadas entre esse tipo de funções.

\subsubsection{Definição de Funções Baseadas em Caso}

Definição 3. Uma função linear $f$ sobre $\vec{Y}$ é definida por

$$
f(\vec{y})=a_{0}+a_{1} y_{1}+a_{2} y_{2}+\ldots+a_{m} y_{m}=\sum_{i=0}^{m} a_{i} y_{i}
$$

em que $a_{i}$ são constantes e $y_{0}=1$ é usado para simplificar a notação.

Definição 4. Um função polinomial $f$ sobre $\vec{Y}$ é definida por

$$
f(\vec{y})=\sum_{i=0}^{n_{p}} m o_{i}(\vec{y})
$$

tal que cada $m o_{i}$ é um monômio sobre $\vec{Y}$ definido por

$$
m o_{i}(\vec{y})=a y_{1}^{b_{1}} y_{2}^{b_{2}} \ldots y_{m}^{b_{m}}=a \prod_{i=0}^{m} y_{i}^{b_{i}},
$$

em que $a$ e cada $b_{i}$ são constantes. 
Definição 5. Um caso $\phi_{i}(\vec{x}, \vec{y})$ é uma fórmula lógica sobre as variáveis $\vec{X}$ e $\vec{Y}$. Dado o conjunto de átomos $\mathbb{A}=\vec{X} \cup(f(\vec{Y})$ op $\mathbb{N}$ ), em que op $\in\{<, \leq,>, \geq\}$ (i.e. o conjunto de variáveis booleanas e inequações lineares e polinomias sobre $\vec{Y}$ ) e o conjunto de conectivos lógicos $\Omega=\{\neg, \wedge, \vee, \rightarrow, \leftrightarrow\}$, uma fórmula lógica em lógica proposicional é definida recursivamente pelo seguinte conjunto de regras:

1. Qualquer elemento do conjunto $\mathbb{A}$ é uma formula lógica.

2. Se $\alpha$ é uma fórmula, então $\neg \alpha$, é uma fórmula.

3. Se $\alpha$ e $\beta$ são fórmulas, então $(\alpha \omega \beta) \mid \omega \in \Omega$ são fórmulas.

4. Nada mais é uma fórmula.

Qualquer fórmula bem formada em lógica proposicional pode ser expressa em Forma Normal Disjuntiva (FND). Uma fórmula em FND é uma fórmula composta de disjunções( $\vee$ ) de cláusulas conjuntivas. Uma cláusula conjuntiva é formada por átomos de $\mathbb{A}$ conectados somente pelos conectivos de negação $(\neg)$ e conjunção $(\wedge)$.

Será assumido daqui para frente que qualquer caso $\phi(\vec{x}, \vec{y})$ apresentado é definido em FND, essa organização é necessária para uma representação eficiente das funções definidas em casos.

Definição 6. Uma função baseada em casos $f(\vec{x}, \vec{y})$ é definida por:

$$
f(\vec{x}, \vec{y})= \begin{cases}\phi_{1}(\vec{x}, \vec{y}): & f_{1}(\vec{y}) \\ \vdots & \vdots \\ \phi_{k}(\vec{x}, \vec{y}): & f_{k}(\vec{y})\end{cases}
$$

Qualquer caso $\phi_{i}$ deve ser disjunto de qualquer outro caso $\phi_{j}$, isto é, $\phi_{i} \neq \phi_{j}, \forall i \neq j$. Além disso, o conjunto de casos $\phi_{i}$ não necessitam cobrir todo o domínio, portanto a função baseada em casos $f$ pode ser uma função parcial e pode ser indefinida para algumas atribuições das variáveis.

\subsubsection{Operações}

Podem ser realizadas operações entre funções baseadas em caso. Entre os operadores de interesse estão os operadores unários: multiplicação escalar $(c \cdot f)$, negação $(-f)$, restrição $\left(\left.f\right|_{\phi}\right)$ e substituição $\left(f_{\sigma}\right)$; e entre os operadores binários estão: adição $(+)$, subtração $(-)$, multiplicação $(\times)$ e maximização $(\max (f, g))$.

As operações unárias de multiplicação escalar e de negação são simplesmente aplicadas em todas as funções $f_{i}, 1 \leq i \leq k$. Por exemplo, seja:

$$
f(\vec{x}, \vec{y})= \begin{cases}y_{1} \geq 0: & 0 \\ y_{1}<0: & y_{1}\end{cases}
$$

A aplicação da multiplicação escalar por 5, resulta em:

$$
5 \cdot f(\vec{x}, \vec{y})=5 \cdot\left\{\begin{array}{ll}
y_{1} \geq 0: & 0 \\
y_{1}<0: & y_{1}
\end{array}= \begin{cases}y_{1} \geq 0: & 5 \cdot 0 \\
y_{1}<0: & 5 \cdot y_{1}\end{cases}\right.
$$

As operações aritméticas binárias (adição, subtração e multiplicação) são realizadas em dois passos: (i) aplicando o produto cruzado nas partições lógicas para cada par de casos; e (ii) aplicando a operação correspondente nas partições resultantes dadas por cada par de casos.

Por exemplo, dadas duas funções baseadas em casos $f$ e $g$, a operação de adição é realizada da 
seguinte maneira:

$$
f+g=\left\{\begin{array}{ll}
\phi_{1}: & f_{1} \\
\phi_{2}: & f_{2}
\end{array}+\left\{\begin{array}{ll}
\psi_{1}: & g_{1} \\
\psi_{2}: & g_{2}
\end{array}= \begin{cases}\phi_{1} \wedge \psi_{1}: & f_{1}+g_{1} \\
\phi_{1} \wedge \psi_{2}: & f_{1}+g_{2} \\
\phi_{2} \wedge \psi_{1}: & f_{2}+g_{1} \\
\phi_{2} \wedge \psi_{2}: & f_{2}+g_{2}\end{cases}\right.\right.
$$

Note que algumas partições resultantes das operações aritméticas binárias podem ser inconsistentes (por exemplo, $\phi_{1} \wedge \psi_{1}=x_{1}>0 \wedge x_{1}<0$ ), nesses casos pode-se simplesmente descartar tais partições uma vez que são irrelevantes para o valor da função.

A operação de maximação é realizada combinando os casos de cada função (mesma combinação realizada nas operações aritméticas) e adicionando em cada caso as comparações $f_{i}>g_{i}$ e $f_{i} \leq g_{i}$ para as funções respectivas de cada partição.

Por exemplo, dadas duas funções baseadas em caso $f$ e $g$, a operação de maximização é realizada da seguinte maneira:

$$
\max (f+g)=\max \left(\left\{\begin{array}{ll}
\phi_{1}: & f_{1} \\
\phi_{2}: & f_{2}
\end{array},\left\{\begin{array}{ll}
\psi_{1}: & g_{1} \\
\psi_{2}: & g_{2}
\end{array}\right)= \begin{cases}\phi_{1} \wedge \psi_{1} \wedge f_{1}>g_{1}: & f_{1} \\
\phi_{1} \wedge \psi_{1} \wedge f_{1} \leq g_{1}: & g_{1} \\
\phi_{1} \wedge \psi_{2} \wedge f_{1}>g_{2}: & f_{1} \\
\phi_{1} \wedge \psi_{2} \wedge f_{1} \leq g_{2}: & g_{2} \\
\phi_{2} \wedge \psi_{1} \wedge f_{2}>g_{1}: & f_{2} \\
\phi_{2} \wedge \psi_{1} \wedge f_{2} \leq g_{1}: & g_{1} \\
\phi_{2} \wedge \psi_{2} \wedge f_{2}>g_{2}: & f_{2} \\
\phi_{2} \wedge \psi_{2} \wedge f_{2} \leq g_{2}: & g_{2}\end{cases}\right.\right.
$$

Outra operação importante é a operação de restrição. A operação de restrição restringe a função $f$ para satisfazer uma dada fórmula $\psi$. A restrição é realizada acrescentando a fórmula $\psi$ em cada caso $\phi_{i}$ do seguinte modo:

$$
f \mid \psi= \begin{cases}\phi_{1} \wedge \psi: & f_{1} \\ \vdots & \vdots \\ \phi_{k} \wedge \psi: & f_{k}\end{cases}
$$

O último operador necessário é a substituição. A substituição recebe um conjunto $\sigma$ de variáveis e suas respectivas substituições (funções lineares ou polinomiais). A substituição é realizada substituindo na função $f$ todas as ocorrências das variáveis em $\sigma$ pela sua respectiva substituição.

Por exemplo, dada a função $f$ definida por:

$$
f= \begin{cases}y_{1}>0: & y_{1}^{\prime} \\ y_{1} \leq 0: & y_{1}^{\prime}+y_{2}^{\prime}\end{cases}
$$

e o conjunto $\sigma_{1}=\left\{y_{1}^{\prime}=y_{1}+y_{2}, y_{2}^{\prime}=y_{1}^{2}+4 y_{2}\right\}$, após aplicar a substituição $f_{\sigma_{1}}$ temos:

$$
f_{\sigma_{1}}= \begin{cases}y_{1}>0: & y_{1}+y_{2} \\ y_{1} \leq 0: & y_{1}^{2}+y_{1}+5 y_{2} .\end{cases}
$$

Além disso, o operador de substituição pode definir uma função baseada em casos para $\sigma$. Nesse caso é feita a conjunção dos casos e realizada a respectiva substituição. 
Por exemplo, dada a função f definida na Equação 2.10 e $\sigma_{2}$ definida por:

$$
\sigma_{2}=\left\{y_{1}^{\prime}=\left\{\begin{array}{ll}
y_{1}>10: & y_{1}+y_{2}+100 \\
y_{1} \leq 10 \wedge y_{1}>0: & y_{1}+y_{2} \\
y_{1} \leq 0: & 0
\end{array}\right\}\right.
$$

o resultado da substituição é dado por:

$$
f_{\sigma_{2}}=\left\{\begin{array}{ll}
y_{1}>0 \wedge y_{1}>10: & y_{1}+y_{2}+100 \\
y_{1}>0 \wedge y_{1} \leq 10: & y_{1}+y_{2} \\
y_{1}>0 \wedge y_{1} \leq 0: & 0 \\
y_{1} \leq 0 \wedge y_{1}>10: & y_{1}+y_{2}+100+y_{2}^{\prime} \\
y_{1} \leq 0 \wedge y_{1} \leq 10 \wedge y_{1}>0: & y_{1}+y_{2}+y_{2}^{\prime} \\
y_{1} \leq 0: & 0+y_{2}^{\prime}
\end{array}= \begin{cases}y_{1}>10: \\
y_{1}>0 \wedge y_{1} \leq 10: & y_{1}+y_{2}+100 \\
y_{1} \leq 0: & y_{2}^{\prime} .\end{cases}\right.
$$

Note que algumas das operações com funções baseadas em caso podem aumentar consideravelmente o número de casos necessários para definir uma função (como observado na operação de maximização). Assim uma representação compacta e eficiente de tais funções é necessária. Na seção seguinte são apresentados os Diagramas de Decisão Algébricos Estendidos (XADDs) que servem para este propósito.

\subsection{Diagrama de Decisão Algébrico Estendido}

Um Diagrama de Decisão Algébrico Estendido (eXtended Algebraic Decision Diagram - XADD) é uma estrutura de dados derivada de ADDs que permitem representar funções baseadas em casos.

Definição 7. Um Diagrama de Decisão Algébrico Estendido (XADD) representa uma função baseada em casos $f(\vec{x}, \vec{y})$ como um grafo acíclico dirigido $G=(V, E)$, tal que:

- $V=D \cup L$ em que $D$ é um conjunto de nós de decisões (chamados também de nós internos) que inclui um nó raiz; e $L$ é um conjunto de nós folhas (chamados também de nós terminais). Cada nó terminal $l \in L$ representa uma função de variáveis contínuas $f_{i}(\vec{y})$. Cada nó interno $d \in D$ representa uma variável binária $X_{i}$ ou uma inequação de variáveis contínuas. Além disso, nós internos têm dois nós filhos $v_{0}$ e $v_{1} \in V$, que são a raiz do XADD esquerdo $v_{0_{\text {xadd }}} \mathrm{e}$ direito $v_{1_{\text {xadd }}}$, respectivamente;

- Cada aresta $e \in E$ de um nó $d \in D$ para um nó $v_{0}$ (ou $v_{1}$ ) representa uma atribuição da expressão lógica representada por $d$ para falso (ou verdadeiro).

As definições de ordenação e redução de XADDs são diretamente derivadas das respectivas definições existentes para ADDs, i.e. é assumida uma predefinição de ordem dos casos e essa ordem é estabelecida e mantida ao término que cada operação. Será assumido daqui para frente que todos os XADDs são ordenados e reduzidos.

Um caminho a partir do nó raiz até um nó terminal $l$ representa uma atribuição total ou parcial das expressões lógicas dos nós internos desse caminho, para o qual a função baseada em casos $f(\vec{x}, \vec{y})$ atribuí o valor de $l$. Em outras palavras, ao percorrer o caminho até o nó terminal $l$, é atribuído a cada expressão lógica o respectivo valor falso (ou verdadeiro) quando seguimos de um nó interno para um nó filho $v_{0}$ (ou $v_{1}$ ). 
A Figura 2.3 ilustra o XADD para a seguinte função baseada em casos:

$$
f= \begin{cases}y_{1}+y_{3}>100 \wedge y_{2}+y_{3}>100: & 0 \\ y_{1}+y_{3}>100 \wedge y_{2}+y_{3} \leq 100: & y_{2} \\ y_{1}+y_{3} \leq 100 \wedge y_{2}+y_{3}>100: & y_{1} \\ y_{1}+y_{2}+y_{3}>100 \wedge y_{1}+y_{3} \leq 100 \wedge y_{2}+y_{3} \leq 100 \wedge y_{2}>y_{1}: & y_{2} \\ y_{1}+y_{2}+y_{3}>100 \wedge y_{1}+y_{3} \leq 100 \wedge y_{2}+y_{3} \leq 100 \wedge y_{2} \leq y_{1}: & y_{1} \\ y_{1}+y_{2}+y_{3} \leq 100: & y_{1}+y_{2}\end{cases}
$$

Nessa figura observa-se: (i) o compartilhamento de inequações que são usadas diversas vezes na definição de uma função baseada em casos, como por exemplo a comparação $y_{1}+y_{3} \leq 100$ que é usada nos casos 4 e 5; (ii) um caso conjuntivo na definição de uma função baseada em casos é separado em diversos nós no XADD, como por exemplo o caso $y_{1}+y_{3}>100 \wedge y_{2}+y_{3}>100$ é divido na figura nos nós $y_{1}+y_{3} \leq 100$ e $y_{2}+y_{3} \leq 100$; e (iii) um caminho a partir do nó raiz até um nó folha define um conjunto de atribuições (verdadeiras ou falsas) para os casos definidos nos nós internos do caminho e define o valor retornado pela função baseada em casos. Por exemplo, o caminho que passa pelos nós $y_{1}+y_{3} \leq 100, y_{2}+y_{3} \leq 100$ e $y_{2}$, representa o segundo caso da função $f$ e equivale a atribuição de $y_{1}+y_{3} \leq 100=$ false e $y_{2}+y_{3} \leq 100=$ true sendo que o valor retornado pela função é $y_{2}$.

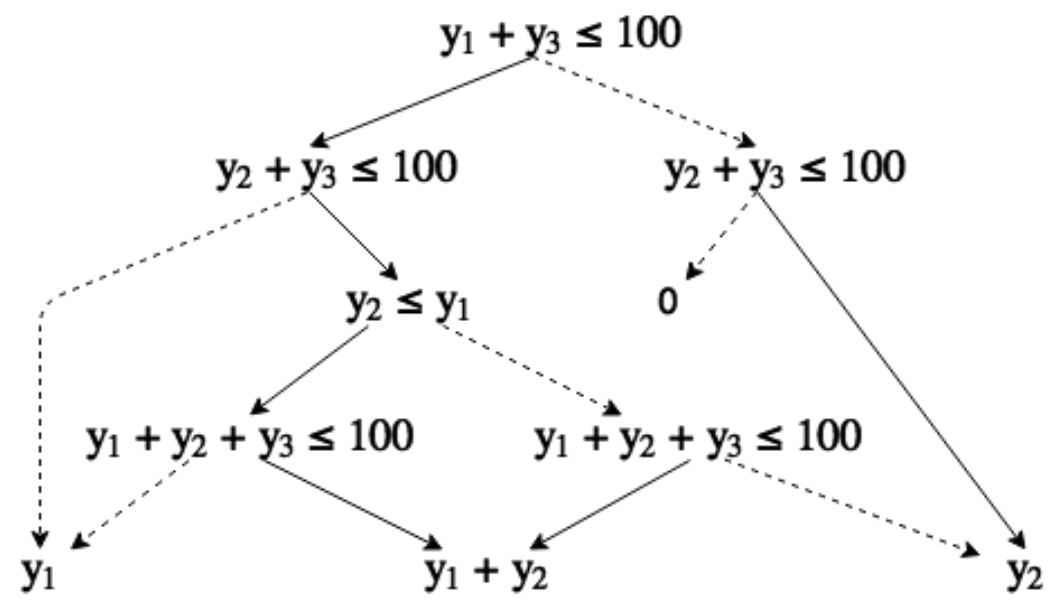

Figura 2.3: Representação da função baseada em casos $f$ da Equação 2.14 por um XADD, em que linhas pontilhadas representam a valoração falsa (0) e as linhas sólidas a valoração verdadeira (1).

Além da representação compacta de funções baseadas em casos, XADDs são capazes de efetuar eficientemente operações binárias como multiplicação $(\otimes)$, soma $(\oplus)$, subtração $(\ominus)$, maximização $(\max (\cdot, \cdot))$ e substituição $\left(f_{\sigma}\right)$.

As operações algébricas de multiplicação, soma e substração ocorrem exatamente da mesma forma do que as respectivas operações para ADDs.

A Figura 2.4 ilustra a operação de substituição na função f (Equação 2.14) substituindo a ocorrência de uma variável em todo o XADD por uma nova expressão, no exemplo foi feita a substituição para $\delta=\left\{y_{3}=10\right\}$. Apesar da operação de substituição ser simples, ela pode desordenar os nós de decisão do XADD em que essa operação foi aplicada. Para resolver esse problema podem ser aplicadas recursivamente as operações de produto e soma, como mostrado nas linhas 5 a 7 do Algoritmo 1 que reordena o XADD $G$. Nesse algoritmo, o identificador do nó $G$ é $G_{i d} ; v_{1_{\text {xadd }}}$ e $v_{0_{\text {xadd }}}$ são os XADDs esquerdo e direito de $G$, respectivamente; e $\mathbb{I}\left[G_{i d}\right]$ é a função indicador para $G_{i d}$, isto é: 


$$
\mathbb{I}\left[G_{i d}\right]= \begin{cases}G_{i d}: & 1 \\ \neg G_{i d}: & 0 .\end{cases}
$$

A Figura 2.5 ilustra um exemplo de um XADD desordenado, sendo que a ordem correta é $y_{1} \leq 10$ e $y_{2} \leq 10$. Para esse exemplo, a Figura 2.6 ilustra a aplicação das operações de produto sobre o XADD $\mathbb{I}\left[G_{i d}\right]$ com o XADD $v_{1_{\text {xadd }}}$ de $G$ (parte superior) e sobre o XADD $\mathbb{I}\left[\neg G_{i d}\right]$ com o XADD $v_{0_{\text {xadd }}}$ de $G$ (parte central). Finalmente, na Figura 2.6 (parte inferior) o XADD ordenado é obtido realizando a operação de soma sobre os dois XADDs obtidos no passo anterior.

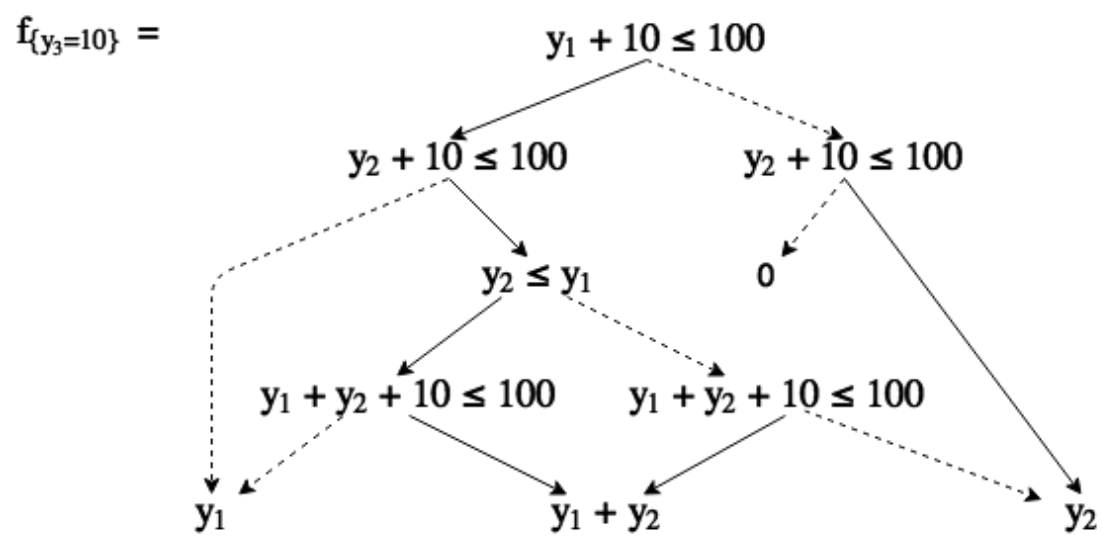

Figura 2.4: Exemplo da operação de substituição para a função $f$ (Equação 2.14) $\operatorname{com} \delta=\left\{y_{3}=10\right\}$.

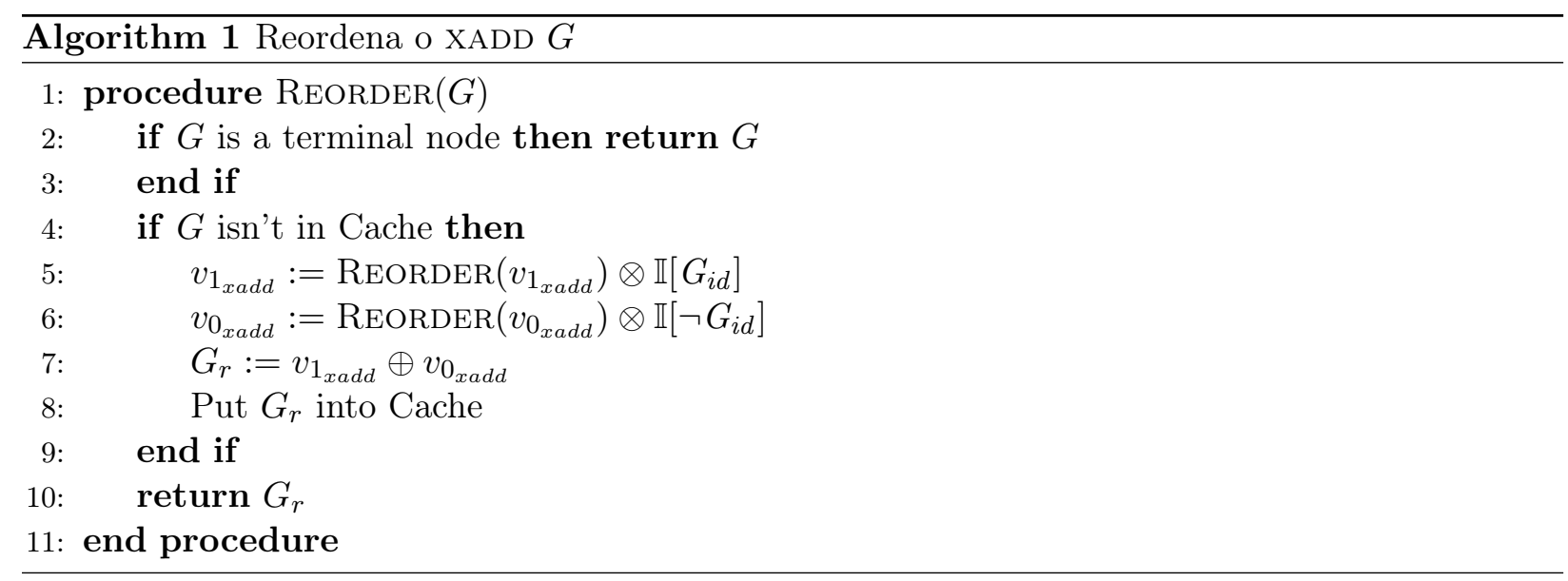

A Figura 2.7 ilustra a operação de maximização entre a função $f$ (Equação 2.14) $\operatorname{com} \delta=\left\{y_{3}=\right.$ $10\}$ e a função $f_{2}$ definida por:

$$
f_{2}= \begin{cases}y_{1}>20: & 2 * y_{1}-y_{2} / 2 \\ y_{1} \leq 20: & 0 .\end{cases}
$$

Na maximização são comparados nós terminais de dois XADDs e mantido aquele de maior valor, além disso a maximização adiciona novos nós de decisão ao XADD resultante. Por exemplo, ao comparar $f_{y_{3}=10}$ e $f_{2}$ na Figura 2.7 foi criado o nó $4 * y_{1} \leq y_{2}$ pois ao seguir as atribuições $y_{1}+10 \leq 100=$ false e $y_{2}+10 \leq 100=$ false da função $f_{y_{3}=10}$ sabemos que a função retorna 0 , porém o valor retornado pela função $f_{2}$ pode ser maior quando $4 * y_{1}>y_{2}$, logo tal comparação deve ser adicionada. 


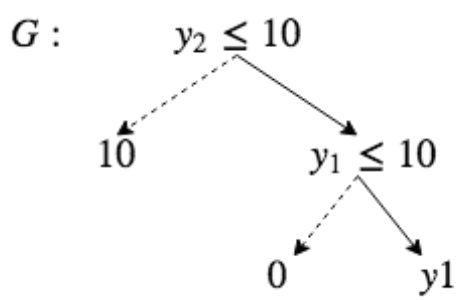

Figura 2.5: Exemplo de um XADD $G$ desordenado.

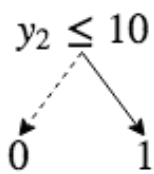

$\otimes$
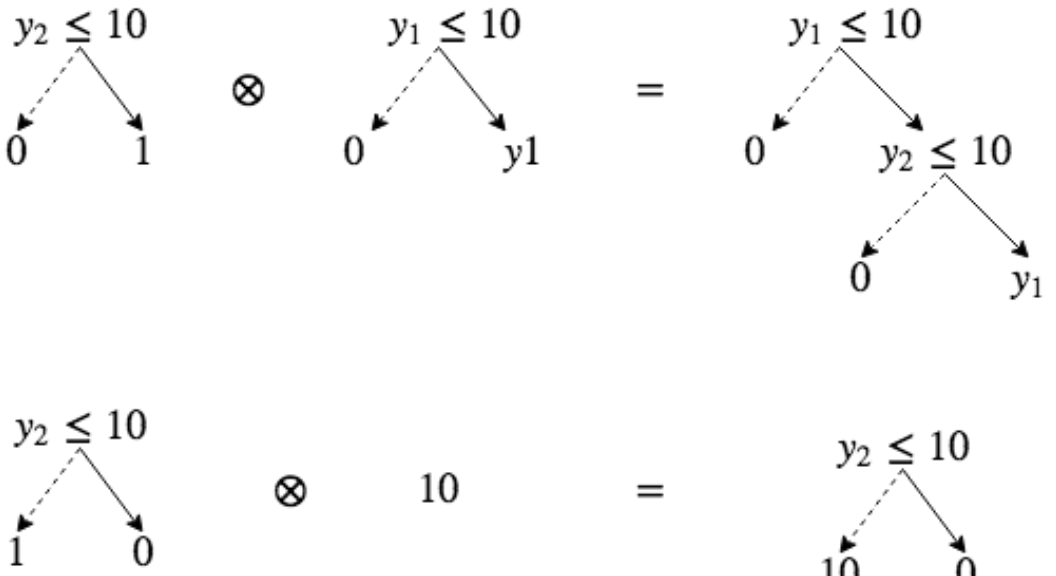

$\otimes$

10
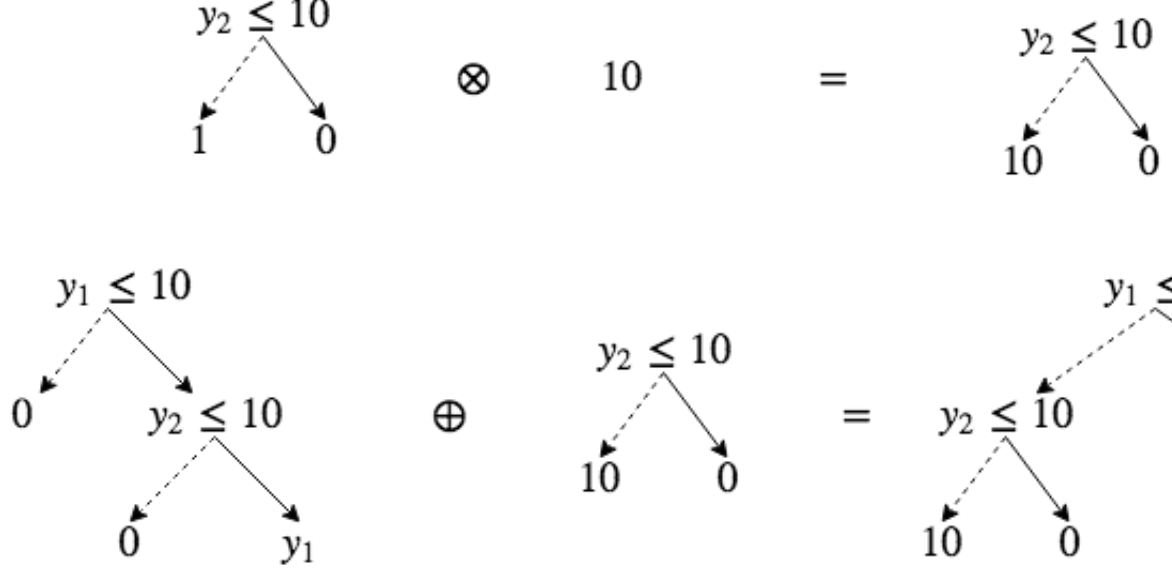

$\oplus$
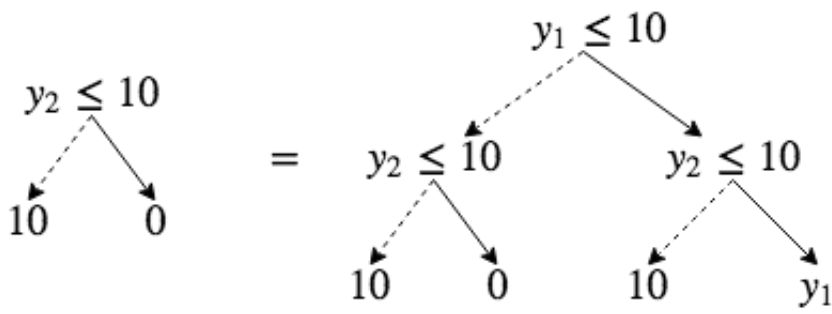

Figura 2.6: Exemplo das operações realizadas respectivamente na linha 5, 6 e 7 do Algoritmo 1 para ordenar $o$ XADD $G$.

Assim, como a operação de substituição a operação de maximização pode desordenar o XADD devido a adição de novos nós de decisão e novamente podemos aplicar o Algoritmo 1 para reordenálo.

XADDs podem ser utilizados para representar de maneira compacta as funções e realizar eficientemente operações para resolver Processos de Decisão Markovianos que incluem variáveis contínuas e discretas, como será visto no Capítulo 6. 


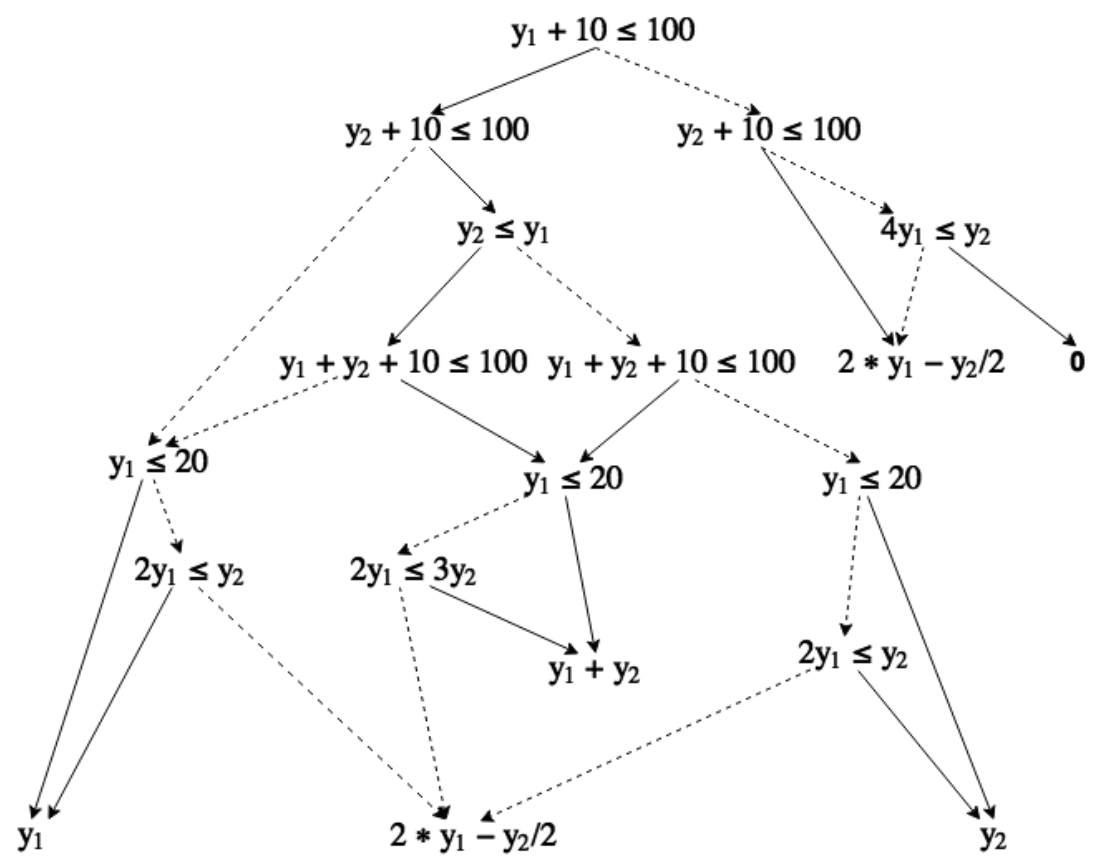

Figura 2.7: Exemplo da operação de maximização para as funções $f_{\left\{y_{3}=10\right\}}$ e $f_{2}$. 


\section{Capítulo 3}

\section{Processos de Decisão Markovianos}

Processo de Decisão Markoviano (MDP) é um modelo matemático para tomada de decisão sequencial comumente utilizado na área de Planejamento Probabilístico. Um MDP modela a sequência de interações de um agente em um ambiente (completamente observável): o agente em cada estágio está em um dado estado do ambiente e escolhe uma ação com um custo associado que o leva a um novo estado de acordo com uma distribuição de probabilidades. Essas interações ocorrem até que o agente atinja um dado estado meta. Neste modelo o objetivo é atingir um estado meta minimizando o custo acumulado esperado obtido pelo agente durante suas interações com o ambiente.

\subsection{Definição Formal}

Definição 8. Um Processo de Decisão Markoviano com estados meta, é chamado de Processo de Decisão Markoviano Dirigido a Metas (Goal-Directed Markov Decision Process - GD-MDP) [BT91, GB13] e é definido por uma tupla $M=\left\langle S, s_{0}, A, T, C, S_{g}\right\rangle$, em que:

- $S$ é um conjunto finito de estados;

- $s_{0} \in S$ é o estado inicial;

- A é um conjunto finito de ações;

- $T: S \times A \times S \rightarrow[0,1]$ é uma função de transição que retorna a probabilidade $T\left(s^{\prime} \mid s, a\right)$ de alcançar o estado $s^{\prime}$ partindo do estado $s$ quando o agente aplica a ação $a$.

- $C: S \times A \rightarrow \mathbb{R}^{+}$é uma função de custo que associa um custo para cada ação $a$ executada em cada estado $s$;

- $S_{g} \subset S$ é um conjunto de estados meta. Cada estado meta é um estado absorvente, isto é $T\left(s_{g} \mid s_{g}, a\right)=1$ e $C\left(s_{g}, a\right)=0, \forall s_{g} \in S_{g}$.

O objetivo de um GD-MDP é atingir um estado meta com o mínimo custo acumulado esperado. A solução para um GD-MDP é representada por uma política $\pi: S \rightarrow A$, que mapeia estados em ações. Assim, dada uma política $\pi$ arbitrária, é possível avaliá-la calculando o custo acumulado esperado de sua execução, isto é:

$$
f^{\pi}(s)=E_{\pi}\left[\sum_{i=0}^{\infty} C\left(s^{i}, \pi\left(s^{i}\right)\right) \mid s^{0}=s\right],
$$

em que $s^{i}$ representa o estado no $i$-ésimo estágio da execução da política $\pi$. 
Pode-se expandir um passo dessa equação afim de explicitar a relação entre duas funções de avaliação sucessoras:

$$
\begin{array}{r}
f^{\pi}(s)=E_{\pi}\left[\sum_{i=0}^{\infty} C\left(s^{i}, \pi\left(s^{i}\right)\right) \mid s^{0}=s\right] \\
f^{\pi}(s)=E_{\pi}\left[C\left(s^{i}, \pi\left(s^{i}\right)\right) \mid s^{0}=s\right]+E_{\pi}\left[\sum_{i=1}^{\infty} C\left(s^{i}, \pi\left(s^{i}\right)\right) \mid s^{1}=s^{\prime} \sim T(\cdot \mid s, \pi(s))\right] \\
f^{\pi}(s)=C(s, \pi(s))+E_{\pi}\left[\sum_{i=1}^{\infty} C\left(s^{i}, \pi\left(s^{i}\right)\right) \mid s^{1}=s^{\prime} \sim T(\cdot \mid s, \pi(s))\right],
\end{array}
$$

em que $T(\cdot \mid s, \pi(s))$ é a distribuição de estados alcançáveis a partir do estado $s$ aplicando a ação dada por $\pi(s)$. Note que o valor esperado no final da Equação 3.2 pode ser escrito em termos da Equação 3.1 em que $s=s^{\prime}$, assim podemos definir a função de avaliação de $\pi$ recursivamente:

$$
f^{\pi}(s)= \begin{cases}C(s, \pi(s))+\sum_{s^{\prime} \in S} T\left(s^{\prime} \mid s, \pi(s)\right) f^{\pi}\left(s^{\prime}\right) & \text { se } s \notin S_{g} \\ 0 & \text { se } s \in S_{g} .\end{cases}
$$

O objetivo de um GD-MDP é encontrar a política ótima $\pi^{*}$ em que $f^{*}=\min _{\pi} f^{\pi}$, que satisfaz a equação de otimalidade de Bellman [Bel57]:

$$
f^{*}(s)= \begin{cases}\min _{a \in A}\left[C(s, a)+\sum_{s^{\prime} \in S} T\left(s^{\prime} \mid s, a\right) f^{*}\left(s^{\prime}\right)\right] & \text { se } s \notin S_{g} \\ 0 & \text { se } s \in S_{g}\end{cases}
$$

GD-MDP é um modelo mais geral que SSP-MDPs (Shortest Stochastic Path MDPs) [Ber95], já que esse último assume duas fortes suposições: (i) existe pelo menos uma política própria $\forall s \in S$ e (ii) todas as políticas impróprias possuem custo esperado infinito. Uma política própria é uma política que leva o agente a um estado meta com probabilidade 1. Qualquer política que não direciona o agente a um estado meta com probabilidade 1 é chamada imprópria. Neste trabalho, são usados GD-MDPs que lidam com risco para modelar problemas como o descrito no Exemplo 1, para o qual não há garantias de políticas próprias.

O espaço de estados de um MDP pode ser representado por um hiper-grafo $G=\langle V, E\rangle$, em que $V$ é o conjunto de vértices e $E$ é o conjunto de arestas. Nesse grafo cada estado $s \in S$ é representado por um vértice, cada ação $a$ e transição $T\left(s^{\prime} \mid s, a\right)$ é representada por uma hiper-aresta no grafo. A Figura 3.1 mostra um exemplo de um MDP, em que os estados $S=\left\{s_{0}, s_{1}, s_{2}, s_{3}\right\}$ são representados por círculos, as ações $a_{i}$ são representadas por quadrados e transições $T\left(s^{\prime} \mid s, a\right)$ são representadas por setas. O conjunto de estados meta é composto por $S_{g}=\left\{s_{3}\right\}$ e a função de custo $C(s, a)$ é descrita no canto superior esquerdo.

\subsection{Algoritmos}

GD-MDPs podem ser resolvidos montando um sistema não-linear de equações a partir da Equação 3.4, porém tal método é complexo e em geral custoso computacionalmente. Assim, outros métodos mais eficientes foram propostos.

O algoritmo Iteração de Valor (VALue ItERATION - VI) [Bel57] é um algoritmo clássico que usa programação dinâmica e é síncrono (i.e. todos os estados $s$ são atualizados em cada iteração). Esse algoritmo clássico serviu como base para diversos outros algoritmos mais eficientes para solucionar GD-MDPs, entre esses destaca-se o algoritmo Iteração de Valor Topológico (Topological VALuE ITERATION - TVI) [DG07]. 


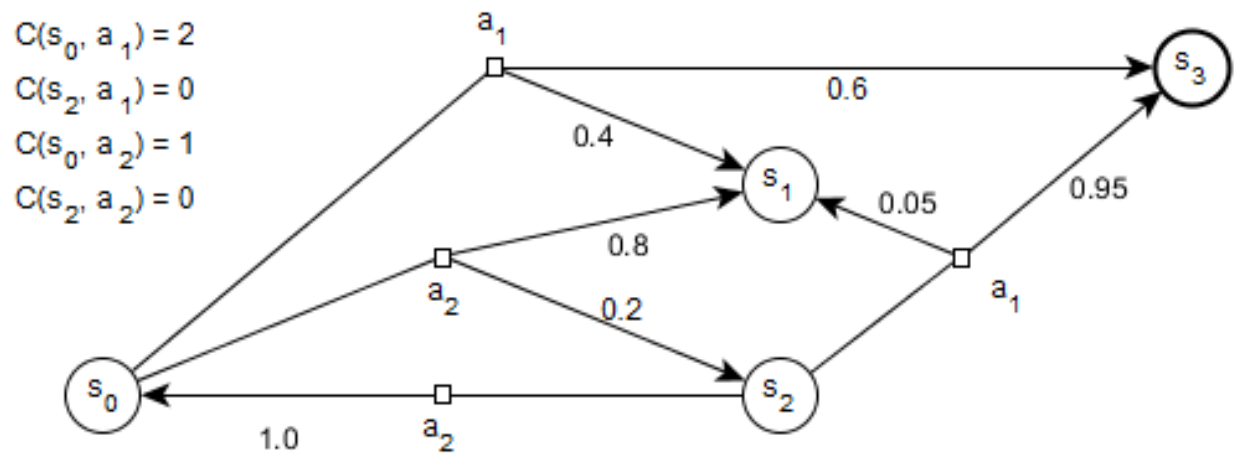

Figura 3.1: Exemplo de um MDP. Transições são denotadas por setas, ações são denotadas por quadrados, estados são denotados por círculos e o custo de cada par estado-ação é mostrado no canto superior-esquerdo.

\subsubsection{Iteração de Valor}

O algoritmo Iteração de Valor (VI, Algoritmo 2) encontra uma política ótima ou $\epsilon$-ótima. $\mathrm{O}$ algoritmo VI começa inicializando $f^{0}$ com um valor arbitrário inicial, por exemplo 0 (Linha 2 do Algoritmo 2). Note que na Equação $3.4 f^{*}(s)=0, \forall\left(s \in S_{g}\right)$, portanto $f^{0}(s)$ deve ser inicializado com valor zero para todos os estados $s \in S_{g}$. VI atualiza iterativamente o valor estimado realizando atualizações de Bellman (Linhas 6 a 10 do Algoritmo 2) como segue:

$$
f^{i+1}(s)=\min _{a \in A}\left[C(s, a)+\sum_{s^{\prime} \in S} T\left(s^{\prime} \mid s, a\right) f^{i}\left(s^{\prime}\right)\right],
$$

em que $i$ é a i-ésima iteração. Essas atualizações são feitas para todos os estados $s \in S$ em cada iteração até que o valor de duas iterações subsequentes atinja um erro mínimo $\epsilon$ (Linhas 4 a 12 do Algoritmo 2). O erro de uma atualização é definido por $\delta=\max _{s \in S}\left|f^{i+1}(s)-f^{i}(s)\right|$ (Linha 8 do Algoritmo 2). Finalmente, a política $\epsilon$-ótima pode ser extraída (Linha 13 do Algoritmo 2) por:

$$
\pi(s)=\underset{a \in A}{\arg \min }\left[C(s, a)+\sum_{s^{\prime} \in S} T\left(s^{\prime} \mid s, a\right) f\left(s^{\prime}\right)\right], \forall(s \in S) .
$$

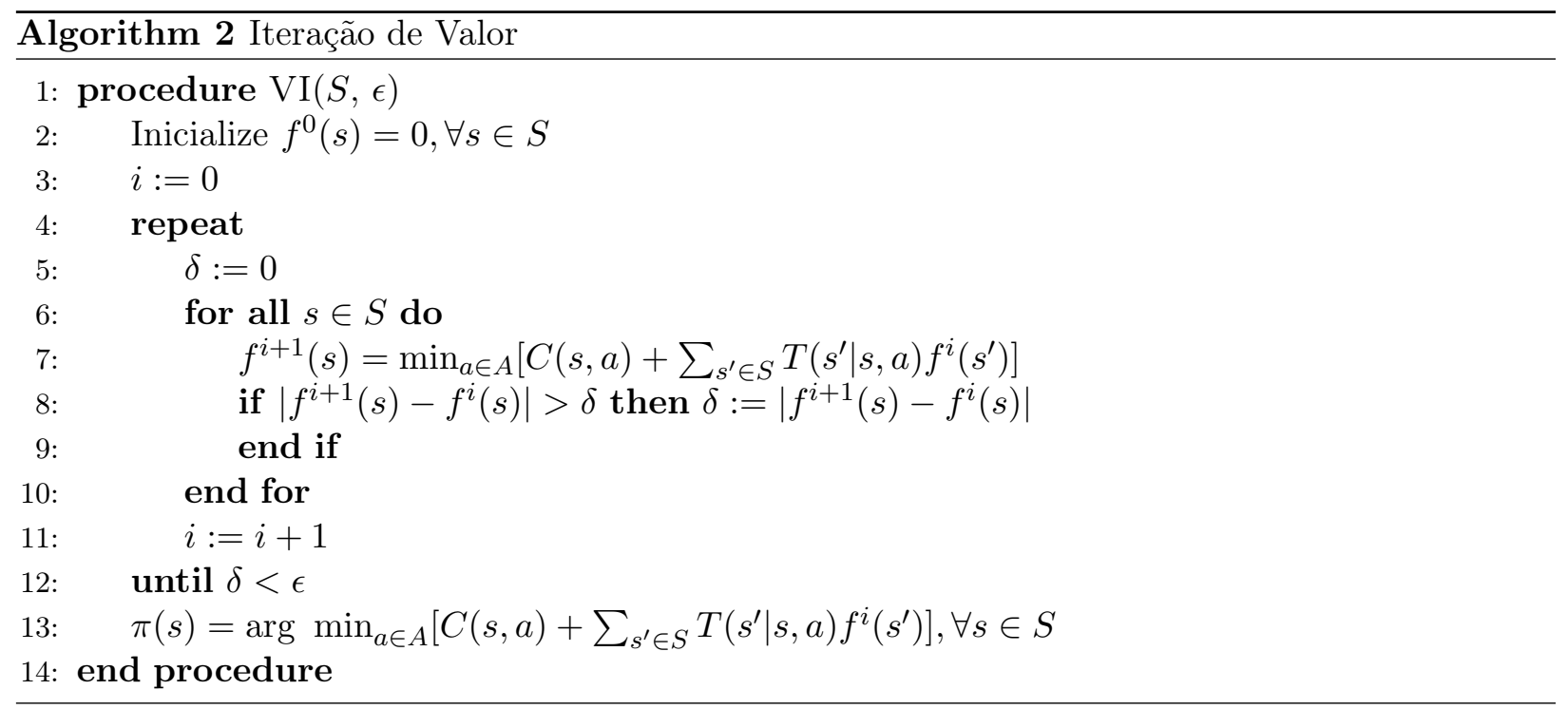




\subsection{Iteração de Valor Topológico}

O algoritmo de Iteração de Valor Topológico (TVI, Algoritmo 3) é um algoritmo assíncrono, i.e., apenas um subconjunto de $S$ é atualizado em cada iteração. O algoritmo TVI é baseado no algoritmo VI e trabalha com componentes fortemente conexas.

Definição 9. Dado um grafo $G=\langle V, A\rangle$, um caminho $h_{\text {path }}=\left\{v_{1}, v_{2}, \ldots, v_{k}\right\}$ é um conjunto de vértices que começa no estado $v_{1}$ e termina no estado $v_{k}$, tal que, para cada par de vértices subsequentes $v_{i}$ e $v_{i+1}$ existe uma aresta $\left(v_{i}, v_{i+1}\right) \in A$.

Definição 10. Um grafo direcionado (ou dirigido) $G=\langle V, A\rangle$ é fortemente conexo quando para qualquer par de vértices $v, u \in V$, tal que $v \neq u$, existe um caminho que conecta $v$ a $u$ e um que conecta $u$ a $v$.

Definição 11. Uma Componente Fortemente Conexa (Strongly Conneceted Components - SCC) em um grafo direcionado $G$ é um subgrafo $G^{\prime}$ tal que $G^{\prime}$ é um grafo fortemente conexo.

O algoritmo TVI começa particionando o conjunto de estados $S$ em sCCs, resultando em um grafo acíclico dirigido $G^{\prime \prime}=\langle Y, A\rangle$. Nesse grafo, cada vértice correspondem a um $\operatorname{sCC} Y_{j} \in Y$, que também é chamado de meta-estado.

A Figura 3.2 mostra o grafo acíclico dirigido $G^{\prime \prime}$ para o exemplo da Figura 3.1, em que os meta-estados (SCCs) $Y_{j}$ são representados por retângulos arredondados. Note que os estados $s_{0} \mathrm{e}$ $s_{2}$ fazem parte de um componente fortemente conexo pois existe um caminho que conecta $s_{0}$ a $s_{2}$ e um caminho que conecta $s_{2}$ a $s_{0}$.

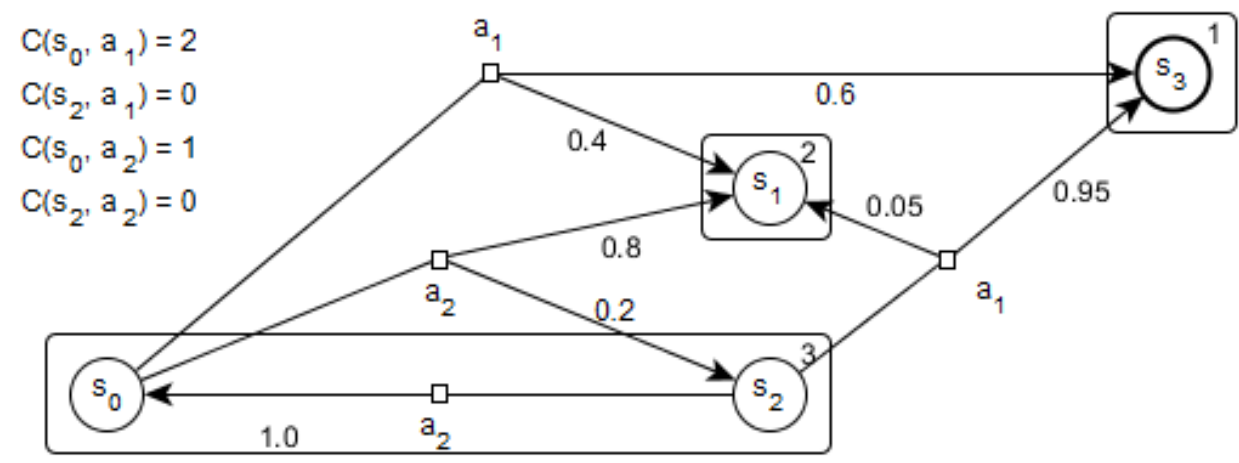

Figura 3.2: $\mathrm{sCC} s$ (retângulos arredondados) para o exemplo de MDP da Figura 3.1

O algoritmo TVI obtêm o grafo acíclico dirigido $G^{\prime \prime}$ de SCCs a partir do estado inicial (Linha 2 do Algoritmo 3), por exemplo, aplicando o algoritmo de Tarjan [Tar71]. Em sequência, o grafo $G^{\prime \prime}$ é percorrido em ordem topológica reversa e cada meta-estado $Y_{j}$ é atualizado executando o algoritmo de Iteração de Valor para todo $s \in Y_{j}$ (Linhas 3-5 do Algoritmo 3).

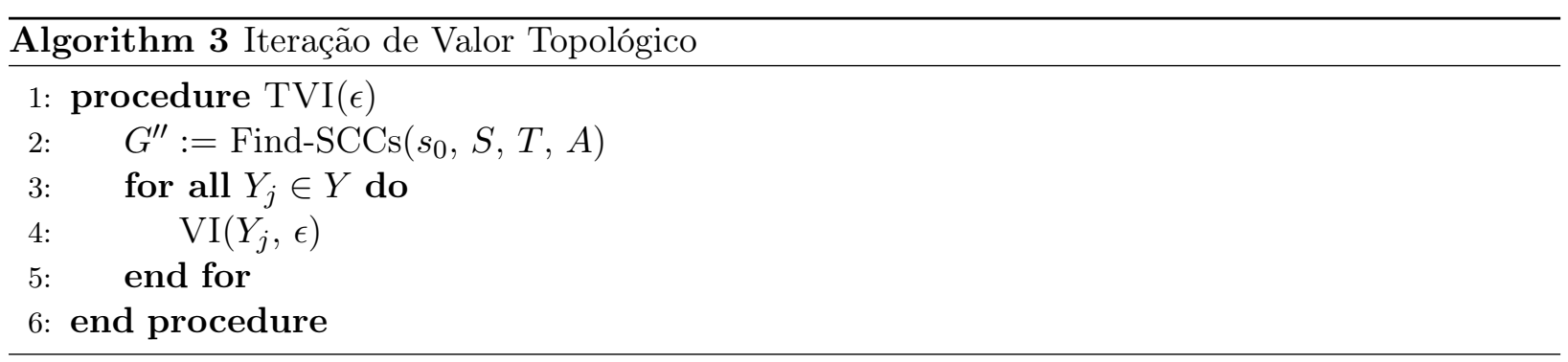

Apesar do Algoritmo vi (chamado na Linha 4 do Algoritmo 3) não necessitar de alterações, a 
equação de atualização utilizada pelo algoritmo vi dado uma SCC $Y_{j}$ é dada por:

$$
f^{i+1}(s)= \begin{cases}\min _{a \in A}\left[C(s, a)+\sum_{s^{\prime} \in S} T\left(s^{\prime} \mid s, a\right) f^{*}\left(s^{\prime}\right)\right] & \text { se } s^{\prime} \notin Y_{j} \\ \min _{a \in A}\left[C(s, a)+\sum_{s^{\prime} \in S} T\left(s^{\prime} \mid s, a\right) f^{i}\left(s^{\prime}\right)\right] & \text { se } s^{\prime} \in Y_{j},\end{cases}
$$

em que $f^{*}$ é o valor retornado ao término da execução do algoritmo vi executado sobre o conjunto de estados pertencente a $Y_{j-1}$ (SCC atualizado antes). Isso ocorre devido a ordem de atualização realizada (i.e. ordem topológica reversa) ser a ordem ótima de atualização [DG07]. Portanto, estados que não pertencem a mesma SCC já possuem a respectiva função valor convergida.

Note que para o algoritmo de Iteração de Valor Topológico apenas um subconjunto do espaço de estados (estados em componentes fortemente conexas) é atualizado, pois estados não pertencentes a esse conjunto não são alcançáveis a partir do estado inicial.

A demonstração da corretude do algoritmo é baseada no teorema a seguir que é válido para MDPs.

Teorema 1. (Ordem de atualização ótima [BY10]) Se um MDP é acíclico, então existe uma ordem de atualização ótima. Aplicando a ordem ótima de atualização, a função valor ótima (Equação 3.4) pode ser encontrada com uma única atualização em cada estado.

O Teorema 1 garante que cada SCC $Y_{j}$ encontrado pelo algoritmo necessita de apenas uma única meta-atualização, já que a ordem ótima de atualização para $G^{\prime \prime}$ é a ordem topológica reversa. No exemplo da Figura 3.2 a ordem de atualização é apresentada pelos números no canto superior direito de cada SCC. 


\section{Capítulo 4}

\section{Processos de Decisão Markovianos Fatorados}

No capítulo anterior foram apresentados os Processos de Decisão Markovianos com representação de estados enumerados, chamado de MDPs enumerativos. Nesta seção são descritos MDPs com representação de estados em termos de variáveis de estado, chamados de MDPs fatorados.

\subsection{Definição Formal}

Definição 12. Um MDP fatorado é definido por uma tupla $M_{f}=\left\langle\vec{X}, A, C_{f}, P, G\right\rangle$ :

- $\vec{X}$ é um vetor de variáveis de estado $\left(X_{1}, \ldots, X_{n}\right)$ de comprimento $n$, em que um estado $s \in S$ é representado pelo vetor de estado $\vec{x}=\left(x_{1}, \ldots, x_{n}\right)$ em que $x_{i} \in\{0,1\}$ é o valor da variável $X_{i}$

- $A$ é um conjunto finito de ações;

- $C_{f}$ é uma função de custo: $\{0,1\}^{n} \times A \rightarrow \mathbb{R}^{+}$, que atribui um valor a cada par estado $\vec{x}$ e ação $a$;

- $P$ é uma função de transição: $S \times A \rightarrow D(S)$, que atribui uma distribuição de probabilidades sobre o conjunto de estados $S$ a cada par estado-ação $(s, a)$. Denotamos $P\left(s^{\prime} \mid s, a\right)=$ $P\left(x_{1}^{\prime}, \ldots, x_{n}^{\prime} \mid x_{1}, \ldots, x_{n}, a\right)$ a densidade de probabilidade associada ao estado $s^{\prime}$ ao aplicar a ação $a$ no estado $s$;

- $G$ é um conjunto finito de vetores de estado $\vec{g} \in\{0,1\}^{n}$. Cada vetor $\vec{g}$ representa um estado meta que é um estado absorvente, isto é, $C_{f}(\vec{g}, a)=0$ e $P\left(\vec{g}^{\prime} \mid \vec{g}, a\right)=1, \forall \vec{g} \in G, \forall a \in A$.

\section{Definição 13. (Rede Bayesiana Dinâmica - DBN [DG93])}

Uma Rede Bayesiana Dinâmica DBN é uma Rede Bayesiana que relaciona variáveis em dois tempos subsequentes, ou seja, cada variável $X_{i}$ no tempo seguinte está relacionada somente com um conjunto de variáveis do tempo atual e seguinte. Denotamos variáveis no tempo atual como $X_{i}$ e variáveis no tempo seguinte como $X_{i}^{\prime}$. Além disso, DBNs representam de forma compacta probabilidades condicionais.

As transições de estados em MDPs fatorados são definidas por Redes Bayesianas Dinâmicas (uma DBN para cada ação $a \in A$ ) em que variáveis de próximo estado $x_{i}^{\prime}$ são condicionadas em um subconjunto de variáveis de estado atual e próximo. Assim, as probabilidades de transição de estados podem ser fatoradas como:

$$
P\left(\vec{x}^{\prime} \mid \vec{x}, a\right)=\prod_{i=1}^{n} \operatorname{Pr}\left(x_{i}^{\prime} \mid p a\left(X_{i}^{\prime}\right), a\right),
$$


em que $\operatorname{Pr}\left(x_{i}^{\prime} \mid p a\left(X_{i}^{\prime}\right), a\right)$ são as probabilidades condicionais definidas na DBN e $p a\left(X_{i}^{\prime}\right)$ é o conjunto de pais de $X_{i}^{\prime}$.

Além disso, não são permitidas dependências mútuas entre duas variáveis $X_{i}^{\prime}$. Usualmente, probabilidades condicionais em uma DBN são representadas por Tabelas de Probabilidade Condicional (Conditional Probability Tables - CPTs). CPTs enumeram todas as possíveis combinações de atribuições de uma dada função, por exemplo da função $\operatorname{Pr}\left(x_{i}^{\prime} \mid p a\left(X_{i}^{\prime}\right), a\right)$, e portanto possuem tamanho exponencial no número de variáveis pais (i.e. assumindo $p a\left(X_{i}^{\prime}\right)=\left\{X_{1}, X_{2}, \ldots, X_{n}\right\}$ o tamanho da CPT será $2^{n}$ ).

A Tabela 4.1 ilustra uma CPT para um exemplo simples em que a probabilidade da variável $X_{1}^{\prime}$ depende de outras três variáveis $\left(X_{1}, X_{2}\right.$ e $\left.X_{3}\right)$. Em uma CPT todas as possíveis atribuições para as variáveis pais $\left(X_{1}, X_{2}\right.$ e $\left.X_{3}\right)$ são enumeradas e para cada atribuição diferente é associada a devida probabilidade $\operatorname{Pr}\left(X_{1}^{\prime}=1 \mid X_{1}, X_{2}, X_{3}\right)$ (última coluna da Tabela 4.1). Note que existem certas independências presentes nas probabilidades condicionais. Por exemplo, as linhas 2 e 3 da Tabela 4.1 possuem a mesma probabilidade e em ambas a atribuição das variáveis $X_{1}=0$ e $X_{2}=0$, logo a atribuição da variável $X_{3}$ se torna independente. Tabelas de Probabilidade Condicional podem ser compactamente representadas por Diagrama de Decisão Algébricas (apresentados no Capítulo 2), capazes de representar algumas das independências entre as variáveis.

Tabela 4.1: Exemplo de uma tabela de probabilidade condicional (CPT)

\begin{tabular}{|c|c|c|c|}
\hline$X_{1}$ & $X_{2}$ & $X_{3}$ & $X_{1}^{\prime}=1$ \\
\hline 0 & 0 & 0 & 0.30 \\
\hline 0 & 0 & 1 & 0.30 \\
\hline 0 & 1 & 0 & 0.95 \\
\hline 0 & 1 & 1 & 0.10 \\
\hline 1 & 0 & 0 & 0.95 \\
\hline 1 & 0 & 1 & 0.95 \\
\hline 1 & 1 & 0 & 0.20 \\
\hline 1 & 1 & 1 & 0.15 \\
\hline
\end{tabular}

A função de otimalidade para MDPs enumerativos (Equação 3.4) pode ser reescrita para MDPs fatorados como:

$$
f^{*}(\vec{x})=\min _{a \in A}\left[C_{f}(\vec{x}, a)+\sum_{\vec{x}^{\prime} \in\{0,1\}^{n}} P\left(\vec{x}^{\prime} \mid \vec{x}, a\right) f^{*}\left(\vec{x}^{\prime}\right)\right],
$$

em que $P$ é definido pela Equação 4.1 .

\section{$4.2 \quad$ Algoritmo SPUDD}

O algoritmo SPUDD (Stochastic Planning using Decision Diagrams) [HSAHB99] é baseado no algoritmo de Iteração de Valor, porém este utiliza ADDs para encontrar uma solução de modo eficiente. O algoritmo SPUDD (Algoritmo 4) encontra a política ótima ou $\epsilon$-ótima atualizando iterativamente o valor estimado:

$$
f^{i+1}(\vec{x})=\min _{a \in A}\left[C_{f}(\vec{x}, a) \oplus \sum_{\vec{x}^{\prime} \in\{0,1\}^{n}} P\left(\vec{x}^{\prime} \mid \vec{x}, a\right) f^{i}\left(\overrightarrow{x^{\prime}}\right)\right],
$$

em que $i$ é a i-ésima iteração. Devido ao pressuposto de independência realizado na DBN, podemos fatorar a Equação 4.3 em:

$$
f^{i+1}(\vec{x})=\min _{a \in A}\left[C_{f}(\vec{x}, a) \oplus \sum_{\overrightarrow{x^{\prime}}} \bigotimes_{j=1}^{n} \operatorname{Pr}\left(x_{j}^{\prime} \mid p a\left(X_{j}^{\prime}\right), a\right) f^{i}\left(\overrightarrow{x^{\prime}}\right)\right] .
$$


O algoritmo SPUDD (Algoritmo 4) realiza o cálculo da Equação 4.4 representando a função valor $f$, a função de custo $C_{f}$ e as probabilidades condicionais $\operatorname{Pr}$ em ADDs. Note que apenas são necessárias as operações de ADDs de multiplicação, soma, marginalização e minimização. Além disso, o cálculo da probabilidade conjunta é realizada por meio do algoritmo de eliminação de variáveis, portanto a marginalização ocorre nas variáveis de estado $X_{j}^{\prime}$ no lugar de ocorrer sobre todos os vetores de estados $\overrightarrow{x^{\prime}}$.

Seja $g(\cdot)$ o ADD que representa a função $g$ para todos os possíveis parâmetros. O algoritmo SPUDD (Algoritmo 4) começa inicializando $f^{0}(\cdot)$ com zero (Linha 3). Em sequência, o algoritmo SPUDD realiza a marginalização para cada variável de estado (Linhas 7-10) do seguinte modo:

$$
\sum_{X_{1}^{\prime}} \operatorname{Pr}\left(X_{1}^{\prime} \mid p a\left(X_{1}^{\prime}\right), a\right) \sum_{X_{2}^{\prime}} \operatorname{Pr}\left(X_{2}^{\prime} \mid p a\left(X_{2}^{\prime}\right), a\right) \ldots \sum_{X_{n}^{\prime}} \operatorname{Pr}\left(X_{n}^{\prime} \mid p a\left(X_{n}^{\prime}\right), a\right) f^{i}(\cdot)^{\prime},
$$

em que $f^{i}(\cdot)^{\prime}$ é a função valor calculada na $i$-ésima iteração porém com todas as variáveis $X_{j}$ transformadas em $X_{j}^{\prime}$. Note que: (i) é assumido para o cálculo da marginalização que não existe dependência mútua entre as variáveis $X_{j}^{\prime}$; (ii) a transformação das variáveis $X_{j}$ em $X_{j}^{\prime}$ têm o intuito de distinguir as variáveis de estado atuais $X_{j}$ das variáveis de estado futuras $X_{j}^{\prime}$; (iii) a cada marginalização feita $\sum_{X_{j}^{\prime}}$, a $j$-ésima variável de estado $X_{j}^{\prime}$ é eliminada, portanto ao término da marginalização apenas variáveis do tempo atual, i.e. $X_{j}$, são representadas pela função obtida.

O valor resultante da marginalização é atribuído a $Q(\cdot, a)$ acrescentado do custo imediato $C_{f}(\cdot, a)$ (Linha 11). Por fim, a função valor seguinte $f^{i+1}(\cdot)$ é obtida minimizando todas as funções $Q(\cdot, a)$ (Linha 13). Essas iterações ocorrem até que o erro máximo entre duas funções valor subsequentes seja menor que o valor $\epsilon$ (Linha 15).

Finalmente, a política $\epsilon$-ótima (Linha 17) pode ser extraída por:

$$
\pi(\cdot)=\underset{a \in A}{\arg \min } Q(\cdot, a) .
$$

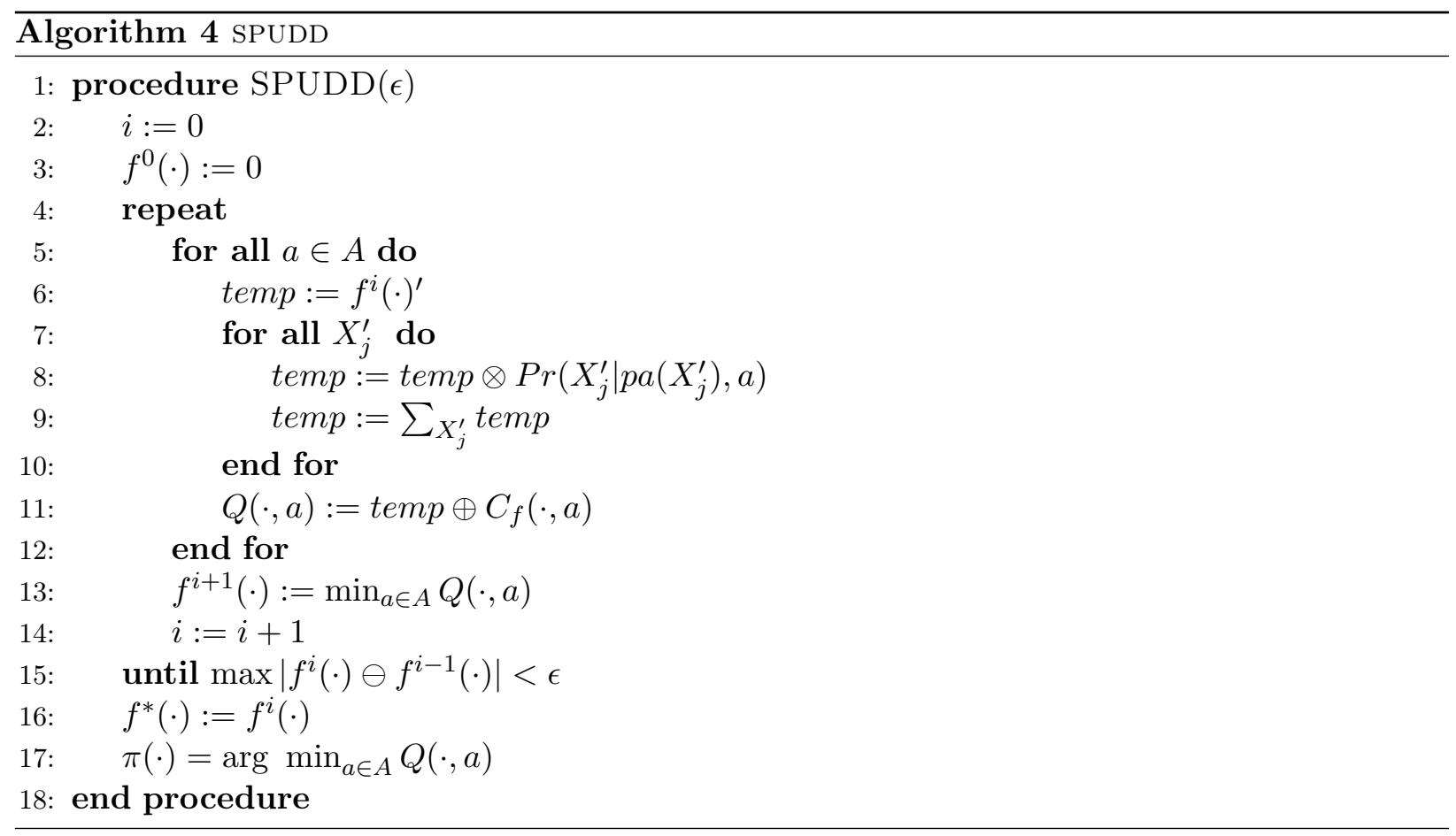




\section{Capítulo 5}

\section{Processos de Decisão Markovianos Sensíveis ao Risco Enumerativos}

Processos de Decisão Markovianos (MDPs) garantem o menor custo acumulado esperado, porém em algumas aplicações a possibilidade de falha (i.e. atingir um estado em que a probabilidade de chegar a meta é zero) ou a possibilidade de obter um custo muito maior do esperado pode ser inaceitável. Afim de tratar essa problemática, diversos outros critérios de otimização foram propostos para MDPs, tais MDPs são chamados de Processos de Decisão Markovianos Sensíveis ao Risco (RS-MDP).

\subsection{Critérios de Sensibilidade ao Risco}

Sensibilidade ao risco pode ser tratada de diversas formas, entre elas [GF15]:

- Otimização da função utilidade do custo acumulado esperado (Equação 3.4) sujeito a um conjunto de restrições adicionais [KKY06].

- Minimização do custo acumulado associado ao cenário do pior caso [GF15, NEG05, DSdB11].

- A substituição da função utilidade do custo acumulado esperado (Equação 3.4) por uma função utilidade exponencial do custo acumulado esperado que permite a modelagem de três possíveis atitudes do agente perante ao risco: aversa, propensa ou neutra [HM72, Pat01, FD16].

- A utilização de uma combinação linear entre variância de custo acumulado e valor do custo acumulado esperado [Mar52] [SKK01];

- A minimização da probabilidade do agente entrar em um estado de falha [GW05].

- A maximização da probabilidade de atingir um estado meta dado um orçamento máximo [YLY98].

Neste trabalho será considerado o último critério para tratamento de sensibilidade ao risco.

\subsection{Definição Formal}

Definição 14. Um Processo de Decisão Markoviano Sensível ao Risco (RS-MDP) [YLY98] pode ser definido como uma tupla $\left\langle M, \theta_{u}\right\rangle$, em que:

- $M$ é um GD-MDP, como definido previamente;

- $\theta_{u} \in \mathbf{R}^{+}$é o custo (ou orçamento) limite definido pelo usuário. 
O objetivo de um RS-MDP é encontrar uma política ótima $\pi^{*}$ que maximize a probabilidade de que o custo acumulado não seja maior que um custo limite $\theta_{u}$ dado pelo usuário [YLY98].

Uma execução $h(\pi)=\left(s^{0}, s^{1}, s^{2}, s^{3}, s^{4}, \ldots\right)$ de uma dada política $\pi$ é uma sequência válida de estados obtidos a partir do estado $s^{0}$, chamada de história de $\pi$. O conjunto $\mathbb{H}$ denotada todas as possíveis histórias de uma política $\pi$ que terminam em um estado meta.

O custo acumulado de uma história $h(\pi)$ é dado por:

$$
c(h(\pi), \theta)=\sum_{t=0}^{\infty} C\left(s^{t}, \pi\left(s^{t}, \theta^{t}\right)\right)
$$

em que $s^{t}$ e $\pi\left(s^{t}, \theta^{t}\right)$ são respectivamente o estado e a ação aplicada no $t$-ésimo passo e $\theta^{t+1}=$ $\theta^{t}-C\left(s^{t}, \pi\left(s^{t}, \theta^{t}\right)\right)$ e $\theta^{0}=\theta$. A probabilidade de uma história $h(\pi)$ ocorrer dado um orçamento $\theta$ é dada por:

$$
\operatorname{Pr}(h(\pi), \theta)=\prod_{t=0}^{\infty} T\left(s^{t+1} \mid s^{t}, \pi\left(s^{t}, \theta^{t}\right)\right) .
$$

em que $\theta^{t}$ está definido como antes. A partir das Equações (5.1) e (5.2), pode-se obter que a probabilidade da política $\pi$ gerar uma historia que começa no estados $s$ e alcança o estado meta sem exceder o orçamento $\theta$ é:

$$
P^{\pi}(s, \theta)=\sum_{h(\pi) \in \mathbb{H}_{\pi}: s^{0}=s, c(h(\pi), \theta) \leq \theta} \operatorname{Pr}(h(\pi), \theta) .
$$

Essa probabilidade é chamada de probabilidade de custo limite da política $\pi$ dado um orçamento $\theta$ a partir de um estado $s$.

Resolver um RS-MDP significa encontrar uma política que maximize a probabilidade de custo limite a partir do estado inicial, dado um orçamento definido pelo usuário, isto é:

$$
\pi^{*}=\arg \max _{\pi} P^{\pi}\left(s_{0}, \theta_{u}\right) .
$$

A Equação 5.4 é chamada de otimização de ponto único - single point optimization, pois essa considera apenas um único orçamento limite $\theta_{u}$.

Seja o conjunto de todos os possíveis orçamentos restantes não negativos, denotado por $\Theta$. Em RS-MDPs, políticas ótimas $\pi^{*}: \mathbb{S}_{a} \rightarrow A$ são estacionárias e determinísticas [HYV14], em que $\mathbb{S}_{a}$ é o conjunto de estados aumentados composto pelo pares $s_{+}=(s, \theta)$ com $s \in S$ e $\theta \in \Theta$.

A probabilidade de custo-limite da política ótima $\pi^{*}$, denotada por $P^{*}(s, \theta)=\max _{\pi} P^{\pi}(s, \theta)$ é o ponto fixo da seguinte Equação de Bellman [YLY98]:

$$
P^{*}(s, \theta)=\max _{a \in A} \sum_{s^{\prime} \in S} \begin{cases}0 & \text { se } C(s, a)>\theta \\ 1 & \text { se } C(s, a)<\theta \text { e } s \in S_{g} \\ T\left(s^{\prime} \mid s, a\right) * P^{*}\left(s^{\prime}, \theta-C(s, a)\right) & \text { se } C(s, a) \leq \theta \text { e } s \notin S_{g}\end{cases}
$$

A Equação 5.5 tem três possíveis casos: (i) $\mathrm{O}$ orçamento restante $\theta$ é insuficiente para executar a ação $a$ no estado $s$, portanto a probabilidade de atingir um estado meta é zero; (ii) o orçamento restante $\theta$ é suficiente para executar a ação $a$ no estado $s$ e $s$ é um estado meta, portanto a probabilidade de atingir um estado meta é 1; e (iii) o orçamento restante $\theta$ é suficiente para executar a ação $a$ no estado $s$ e $s$ é um estado não meta, portanto a probabilidade de atingir um estado meta é dado pela soma do produto da probabilidade de transitar para o estado sucessor $s^{\prime}$ com a probabilidade de custo-limite ótima do estado sucessor, $P^{*}\left(s^{\prime}, \theta-C(s, a)\right)$. Note que o orçamento restante para o estado $s^{\prime}$ é o orçamento restante atual menos o custo de executar a ação $a$ no estado $s$.

A política ótima pode ser extraída aplicando arg $\max _{a}$ no lugar de $\max _{a}$ na Equação 5.5. Note que devido a existência de ciclos de custo zero no espaço de estados aumentado, nem todas as 
soluções de ponto fixo da equação de Bellman são soluções para $P^{*}\left(s_{0}, \theta_{u}\right)$.

O espaço de estados de um RS-MDP pode ser representado por um grafo dirigido, em que cada estado aumentado $(s, \theta) \in \mathbb{S}_{a}$ é representado por um vértice no grafo e cada transição aumentada $T\left(s^{\prime}, \theta-C(s, a) \mid s, \theta, a\right)$ é representada por uma aresta, em que transições aumentadas são definidas como:

$$
T\left(s^{\prime}, \theta-C(s, a) \mid s, \theta, a\right)= \begin{cases}0 & \text { se } C(s, a)>\theta \\ T\left(s^{\prime} \mid s, a\right) & \text { caso contrário. }\end{cases}
$$

Por exemplo, dado $\theta_{u}=2$ e o conjunto de possíveis orçamentos restantes $\Theta=\{0,1,2\}$, podemos gerar o grafo do espaço de estados aumentados do exemplo da Figura 3.1. A Figura 5.1 mostra o grafo resultante para esse exemplo, em que estados aumentados são representados por círculos e transições aumentadas por arcos. Note que o conjunto de estados aumentados é dado por $S \times \Theta$ e são omitidas todas as ações que requererem custo maior que o orçamento disponível em um dado estado aumentado. Por exemplo, para o estado estendido $\left(s_{0}, 1\right)$ duas ações podem ser aplicadas $a_{1}$ com custo 2 e $a_{2}$ com custo 1 . Uma vez que o orçamento disponível nesse estado aumentado é 1 , apenas as transições usando a ação $a_{2}$ são mostradas pois para a ação $a_{1}$ a probabilidade de transição é 0 .

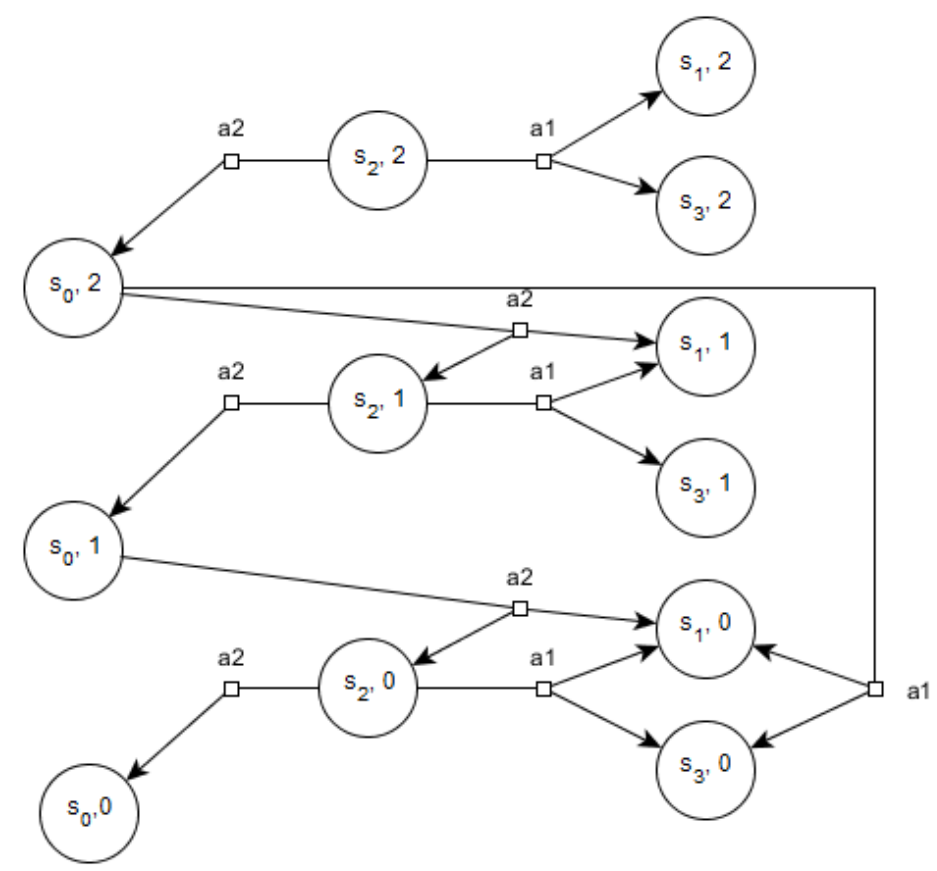

Figura 5.1: Exemplo do espaço de estados aumentado para $\theta_{u}=2$ referente ao MDP da Figura 3.1, em que transições são denotadas por arcos e estados aumentados por círculos.

\subsection{Algoritmos}

São apresentados a seguir o algoritmo Iteração de Valor modificado para solucionar RS-MDPs, chamado de RSVI, e mais dois algoritmos baseados no algoritmo Iteração de Valor Topológico, chamados de TVI-DFS [HYV14] e TVI-DP [HYV14].

\section{Iteração de Valor para RS-MDP}

A probabilidade de custo-limite ótima é um ponto fixo da equação de Bellman, portanto pode ser calculada utilizando o algoritmo de Iteração de Valor Sensível ao Risco (Risk Sensitive Value Iteration - RSVI, Algoritmo 5). O algoritmo RSVI utiliza a seguinte regra de atualização até 
convergência:

$$
P^{i+1}(s, \theta)= \begin{cases}0 & \text { if } C(s, a)>\theta, \\ \max _{a \in A} \sum_{s^{\prime} \in S} T\left(s, a, s^{\prime}\right) P^{i}\left(s^{\prime}, \theta-C(s, a)\right) & \text { if } C(s, a) \leq \theta,\end{cases}
$$

em que

$$
P^{0}(s, \theta)=\left\{\begin{array}{ll}
1 & \text { se } s \in S_{g} \\
0 & \text { se } s \notin S_{g}
\end{array} .\right.
$$

A inicialização acima garante que o algoritmo converge para o ponto fixo ótimo, pois ciclos de custo zero são inicializados com zero de probabilidade. Após a inicialização (Linhas 2 e 3 do Algoritmo 5), o algoritmo BELLMAN_UPDATE é chamado na Linha 4.

O algoritmo Bellman_update (Algoritmo 6) implementa e executa a atualização definida na Equação 5.7 até que o erro seja menor que $\epsilon$ (Linhas 3-11 do Algoritmo 6). Nas linhas 12-14 é obtida a política $\pi$.
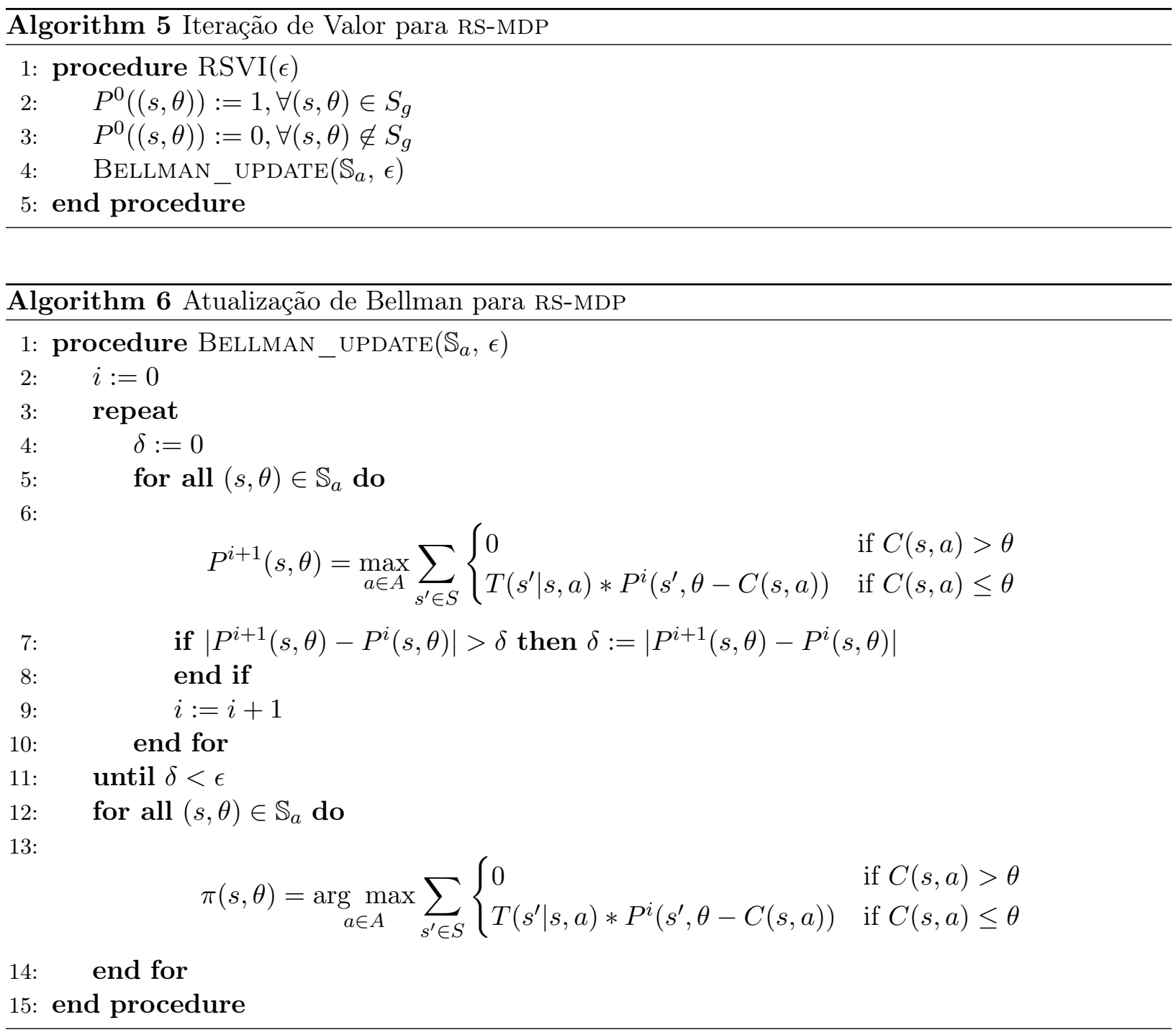

\section{TVI-DFS}

TVI-DFs [HYV14] (Topological Value Iteration - Depth-First Search) é uma adaptação do algoritmo TVI para a solução de RS-MDPs. Em geral, TVI-DFS (Algoritmo 7) segue os mesmos passos do algoritmo TVI (Algoritmo 3), porém com duas importantes modificações: (i) o particionamento do conjunto de estados em SCCs é realizado no conjunto de estados aumentados partindo do estado 
aumentado inicial $\left(s_{0}, \theta_{u}\right)$ (Linha 3 do Algoritmo 7); e (ii) a atualização de cada $\operatorname{SCC} Y_{j}$ do grafo $G$ é feita chamando o algoritmo BELlman_uPdATE_tVi (Linhas 4-6 do Algoritmo 7). O algoritmo BELLMAN_UPDATE_TVI realiza a seguinte operação de atualização até convergência (Linhas 3-11 do Algoritmo 8):

$$
P^{i+1}(s, \theta)=\max _{a \in A} \sum_{s^{\prime} \in S} \begin{cases}0 & \text { se } C(s, a)>\theta \\ T\left(s^{\prime} \mid s, a\right) * P^{*}\left(s^{\prime}, \theta-C(s, a)\right) & \text { se } s_{+}^{\prime} \notin Y_{j}, C(s, a) \leq \theta \\ T\left(s^{\prime} \mid s, a\right) * P^{i}\left(s^{\prime}, \theta-C(s, a)\right) & \text { se } s_{+}^{\prime} \in Y_{j}, C(s, a) \leq \theta,\end{cases}
$$

em que $i$ representa a $i$-ésima iteração, $s_{+}^{\prime}$ representa o estado aumentado $\left(s^{\prime}, \theta-C(s, a)\right)$. Note que é feita uma distinção entre estados aumentados sucessores que pertencem a mesma SCC $Y_{j}$ daqueles que não pertencem. Uma vez que as SCCs são visitadas em ordem topológica, estados aumentados sucessores que não pertencem a mesma SCC do que o estado aumentado atual $(s, \theta)$, já foram atualizados até convergência (caso 2 da Equação 5.9). Porém, estados aumentados que pertencem a mesma SCC que o estado aumentado atual serão atualizados juntos (caso 3 da Equação 5.9). Finalmente o algoritmo BELlMAN_UPDATE_TVI calcula a política (Linhas 12-14 do Algoritmo 8).
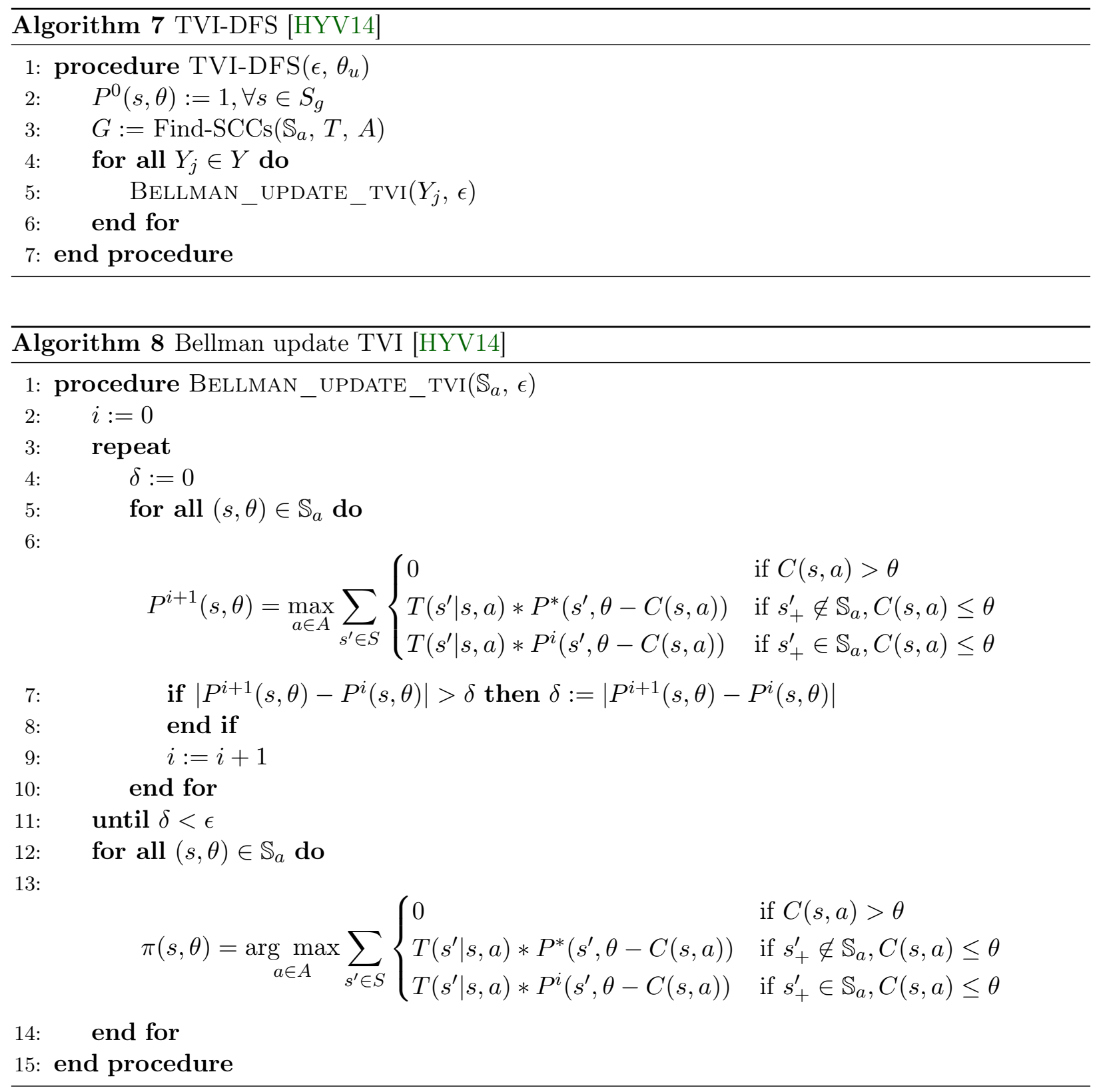

Um esboço da prova de corretude e completude para o algoritmo TVI-DFs é mostrado no Teorema 
2 .

Teorema 2. TVI-DFS é correto e completo [HYV14].

Demonstração. A probabilidade de custo-limite de um estado aumentado $(s, \theta)$ depende somente da probabilidade de custo-limite dos seus estados sucessores $\left(s^{\prime}, \theta^{\prime}\right)$ (veja Equação 5.5). Portanto, a probabilidade de custo-limite de um estado aumentado que pertence a um SCC depende somente da probabilidade de custo-limite de estados aumentados que pertencem a mesma SCC e de SCCs anteriores considerando a ordenação topológica. Já as SCCs são atualizadas em ordem topológica reversa e as probabilidades de custo-limite são corretas após a atualização. Portanto, o algoritmo é correto. O algoritmo é também completo porque cada SCC é atualizada até convergência somente uma única vez.

Na Figura 5.2 são mostradas as SCCs e a ordem de atualização obtidas ao executar o algoritmo TVI-DFS para o exemplo da Figura 5.1, em que o estado aumentado inicial é $\left(s_{0}, 2\right)$ e os estados aumentados meta são $\left(s_{3}, 2\right),\left(s_{3}, 1\right)$ e $\left(s_{3}, 0\right)$.

Os estados são atualizados seguindo a ordem crescente dos valores denotados no canto superior direito de cada retângulo arredondado da Figura 5.2. O estado aumentado $\left(s_{3}, 0\right)$ é primeiramente atualizado até convergência seguindo o caso 3 da Equação 5.9. Note que estados metas são absorventes e inicializados com o valor 1, i.e. a transição $T\left(\left(s_{3}, 0\right) \mid\left(s_{3}, 0\right), a\right)=1$ existe apesar de estar oculta na Figura 5.2 e $P^{*}\left(s_{3}, 0\right)=1$. Em sequência os estados aumentados $\left(s_{1}, 0\right)$ e depois $\left(s_{0}, 0\right)$ são atualizados seguindo o caso 1 da Equação 5.9 pois para esses estados aumentados a execução da ação $a_{1}$ ou $a_{2}$ excederia o orçamento limite disponível. Com esses estados aumentados atualizados, o estado aumentado $\left(s_{2}, 0\right)$ é atualizado seguindo o caso 2 da Equação 5.9 pois o custo das ações aplicáveis nesse estado é 0 . Os demais estados aumentados são atualizados similarmente.

Por fim, as probabilidades de custo-limite para esse exemplo são mostrados na Tabela 5.1. Note que apenas estados em SCCs são atualizados.

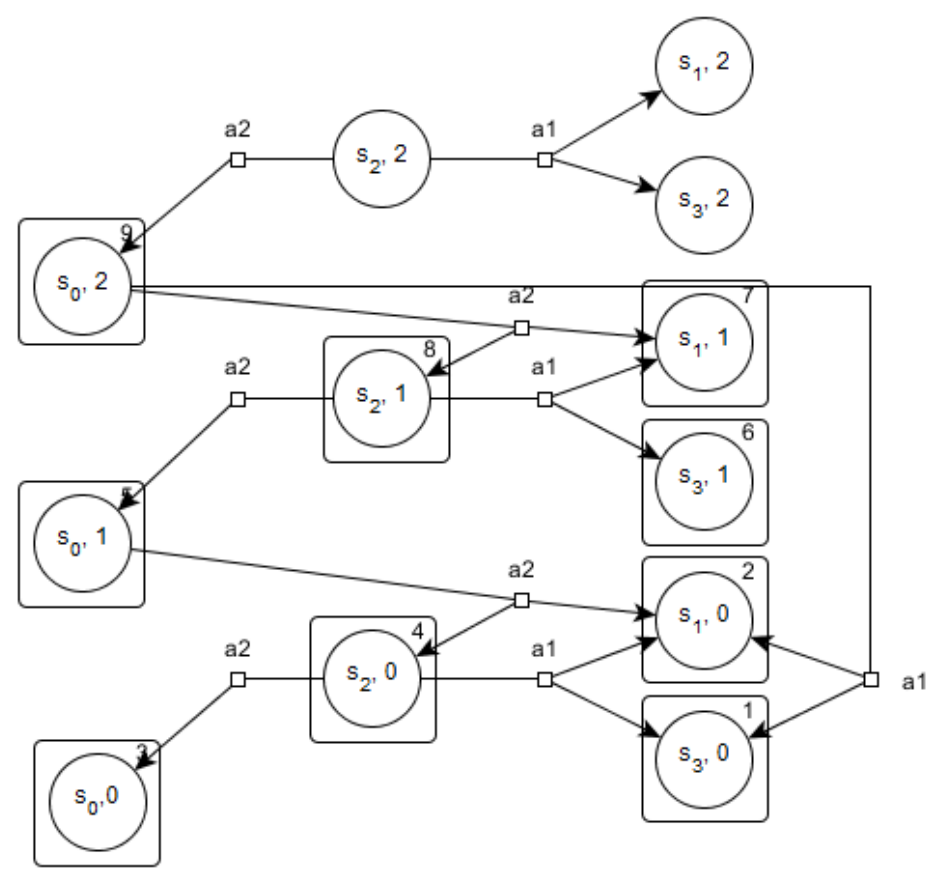

Figura 5.2: Execução do algoritmo TVI-DFS para o exemplo da Figura 5.1, em que SCCs são denotadas por retângulos arredondados. 
Tabela 5.1: Probabilidades de custo-limite calculadas pelo algoritmo TVI-DFS para cada estado aumentado $\left(s_{i}, \theta\right)$ considerando $\theta_{u}=2$

\begin{tabular}{c|l|c|c|c|}
\cline { 2 - 5 } 2 & 0.6 & - & - & - \\
\cline { 2 - 5 } 1 & 0.19 & 0.0 & 0.95 & 1.0 \\
\cline { 2 - 5 } 0 & 0.0 & 0.0 & 0.95 & 1.0 \\
\cline { 2 - 5 } \multicolumn{2}{c|}{$s_{0}$} & $s_{1}$ & $s_{2}$ & $s_{3}$
\end{tabular}

\section{TVI-DP}

TVI-DP [HYV14] (Topological Value Iteration - Dynamic Programming) é também um algoritmo baseado em TVI para a solução de RS-MDPs, porém ao contrário do algoritmo TVI-DF que encontra uma política somente para um custo limite $\theta_{u}$ dado pelo usuário (critério de otimização de ponto único), TVI-DP computa as probabilidades de custo-limite ótimas para todos os $\theta$ s, variando entre 0 até $\theta_{u}$, com incremento de 1 . Esse cálculo é chamado de critério de otimização local estocástica (local stochastic optimization criterion) [YLY98], esse critério é útil quando o estado inicial é desconhecido ou o usuário não possui certeza sobre o custo limite dado. Suponha que em uma usina elétrica deve ser gerada energia com probabilidade acima de $95 \%$ e gostaríamos de limitar o custo gasto. Seguindo o critério de otimização de ponto único seriam necessários diversas tentativas e erros até o valor que desejamos seja encontrado (i.e, $\theta_{u}$ pode ser muito baixo e falhar em encontrar uma política). Seguindo o critério de otimização local estocástica teríamos a possibilidade de selecionar o valor desejado passando um valor superior de $\theta_{u}$. Por exemplo, suponha que exista uma política ótima $\pi_{1}$ somente para o custo limite inicial $\theta_{u}=5$ e uma segunda política ótima $\pi_{2}$ para os custos limites entre $0 \leq \theta \leq 5$. Para o critério de otimização de ponto único as políticas são indistinguíveis, i.e. $P^{\pi_{1}}\left(s_{0}, \theta_{u}\right)=P^{\pi_{2}}\left(s_{0}, \theta_{u}\right)$, mas para o critério de otimização local somente a segunda é uma política ótima.

O algoritmo TVI-DP (Algoritmo 9) utiliza também a Equação 5.9 e em geral segue os mesmos passos do algoritmo TVI-DFS (Algoritmo 7), porém com quatro importantes modificações:

- O particionamento do conjunto de estados aumentados em SCCs é realizado partindo de todos os estados aumentados meta, i.e. $(s, \theta) \mid s \in S_{g}$ e $\theta=0$. Além disso, o particionamento é feito considerando transições transpostas $T^{\prime}$, isto é para cada transição $T\left(s^{\prime} \mid s, a\right)>0$ é criada uma transição no sentido contrário, $T^{\prime}\left(s \mid s^{\prime}, a\right)=T\left(s^{\prime} \mid s, a\right)$ (Linha 4 do Algoritmo 9).

- Os valores entre 0 e $\theta_{u}$ são percorridos (Linhas 5-13 do Algoritmo 9).

- Antes de atualizar cada SCC, os valores de $P(s, \theta-1)$ e $\pi(s, \theta-1)$ para todos os estados $s \in S$ são copiados para os respectivos estados com orçamento corrente $\theta$ (Linhas 6-9 do Algoritmo 9)). Note que essa cópia dos valores têm duas importantes propriedades. Primeiro, essa cópia garante uma inicialização admissível para os valores $P(s, \theta)$, consequentemente a convergência é agilizada quando comparada com o algoritmo TVI-DP que não realiza nenhuma inicialização. A segunda característica é a garantia de que estados que não se encontram em nenhuma SCC para o custo limite $\theta$ devem atingir um estado meta com a mesma probabilidade e política do que o calculado para $\theta-1$.

- As sCCs em função de um dado $\theta$ (denotada por $Y^{\theta}$ ) são percorridas em ordem crescente de $\theta$, assim como na ordem topológica reversa (Linhas 10-12 do Algoritmo 9).

O esboço da prova de corretude para o algoritmo TVI-DP é apresentado no Teorema 3.

Teorema 3. TVI-DP é correto e completo [HYV14].

Demonstração. Para estados aumentados que estão em SCCs, as probabilidades de custo-limite são corretas pelo mesmo argumento dado no Teorema 2. Para cada estado aumentado $(s, \theta)$ que não está em uma SCC, a política que direciona até um estado aumentado meta $\left(s_{g}, \theta_{g}\right)$ será a mesma política daquele do estado aumentado $(s, \theta-1)$ até $\left(s_{g}, \theta_{g}-1\right)$. A probabilidade de custo limite 
para cada estado aumentado $\left(s^{\prime}, \theta^{\prime}\right)$ visitado seguindo a política também será igual à dos estados aumentados $\left(s^{\prime}, \theta^{\prime}-1\right)$. Portanto, copiar as probabilidades e políticas (linhas 7 e 8 do Algoritmo 9) é correto. Consequentemente, o algoritmo é correto. O algoritmo também é completo, pois cada SCC é atualizada somente uma única vez até convergência e cada estado aumentado que não está em uma SCC tem sua probabilidade atualizada somente uma vez.

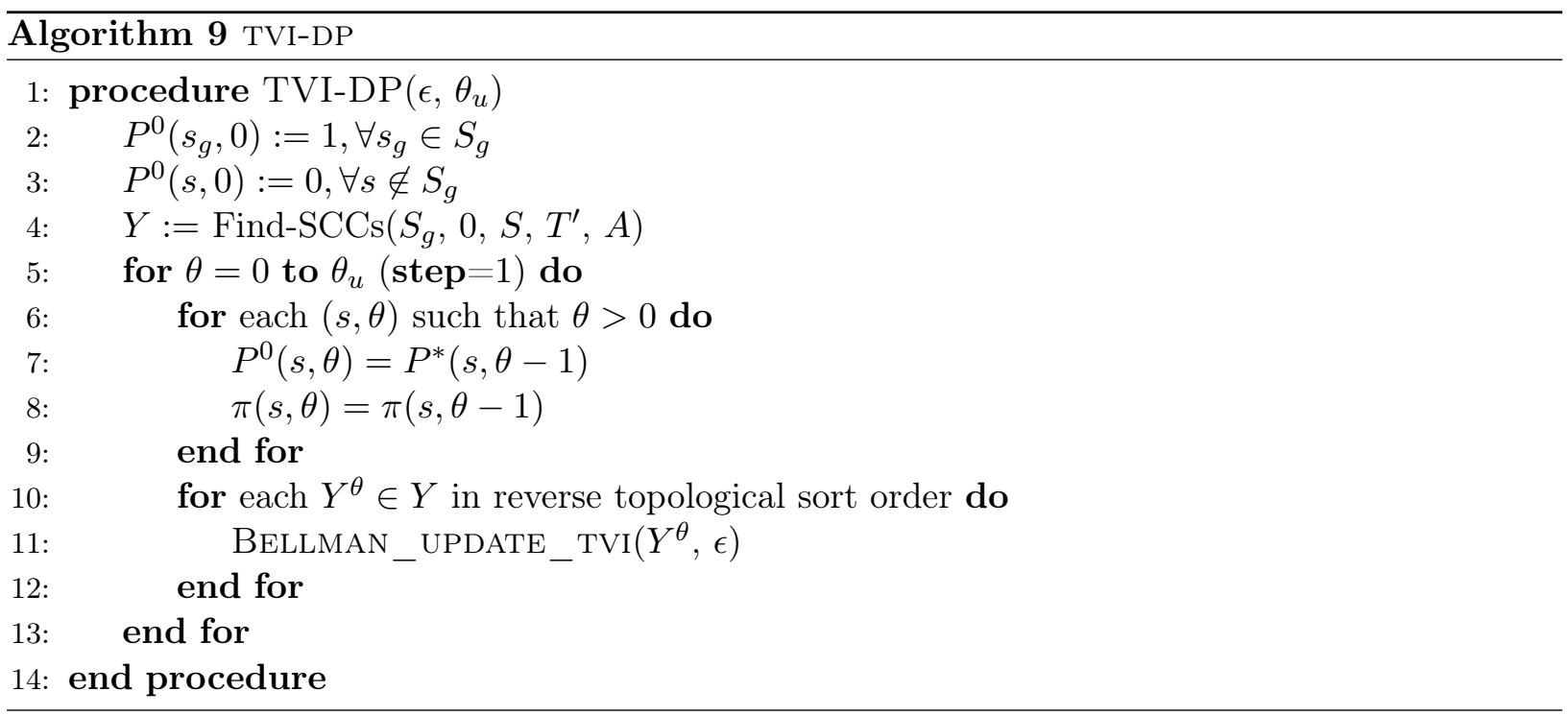

Na Figura 5.3 são mostradas as SCCs e a ordem de atualização obtidas ao executar o algoritmo TVI-DP para o exemplo da Figura 5.1. $P^{0}$ é inicializado com 0 para todos os estados aumentados, com exceção de $P^{0}\left(s_{3}, 0\right)$ que é inicializado com 1 , por $s_{3}$ ser estado meta. A seguir TVI-DP atualiza todos as SCCs com $\theta=0$, que são $\left(s_{3}, 0\right)$ e $\left(s_{2}, 0\right)$, em ordem topológica reversa ${ }^{1}$ utilizando o algoritmo BELlMAN_uPdATE_TVI em cada SCC. A primeira linha da Tabela 5.2 mostra as probabilidades de custo-limite ótima obtidas após realizar essas atualizações. A seguir TVI-DP atualiza todos as SCCs com $\theta=1$, que são $\left(s_{0}, 1\right)$ e $\left(s_{2}, 1\right)$. Similarmente os demais estados aumentados são atualizados.

As probabilidades de custo-limite ótima para esse exemplo são mostrados na Tabela 5.2. Note que TVI-DP atualiza todos os estados independente de pertencerem a uma SCC ou não.

Tabela 5.2: Probabilidades de custo-limite calculadas pelo algoritmo TVI-DP para cada estado aumentado $\left(s_{i}, \theta\right)$ considerando $\theta_{u}=2$

\begin{tabular}{c|l|c|c|c|}
2 & 0.6 & 0.0 & 0.95 & 1.0 \\
\cline { 2 - 5 } 1 & 0.19 & 0.0 & 0.95 & 1.0 \\
\cline { 2 - 5 } 0 & 0.0 & 0.0 & 0.95 & 1.0 \\
\cline { 2 - 5 } \multicolumn{2}{c|}{$s_{0}$} & $s_{1}$ & $s_{2}$ & $s_{3}$
\end{tabular}

\footnotetext{
${ }^{1}$ A ordem está no canto superior direito dos retângulos arredondados na Figura 5.3
} 


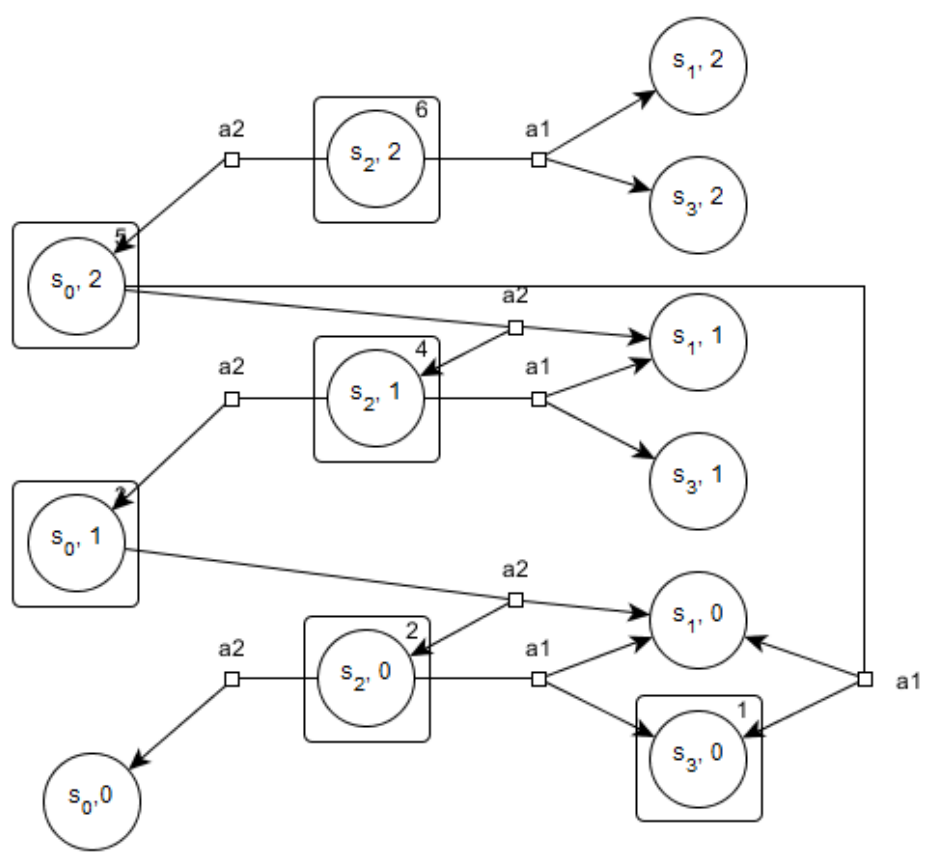

Figura 5.3: Execução do algoritmo TVI-DP para o exemplo da Figura 5.1, em que SCCs são denotadas por retângulos arredondados. 


\section{Capítulo 6}

\section{Processos de Decisão Markovianos Híbridos}

Neste capítulo é apresentada uma extensão de Processos de Decisão Markovianos Fatorados, chamado de Processos de Decisão Markovianos Híbridos (HMDPs). No HMDP estados são representados por uma combinação de variáveis contínuas e booleanas. Além disso, é apresentado o algoritmo de Programação Dinâmica Simbólica (Symbolic Dynamic Programming - SDP) que soluciona HMDPs utilizando uma extensão dos Diagramas de Decisão Algébricos, o Diagrama de Decisão Algébrico Estendido (descrito na Seção 2.3).

Neste capítulo é utilizada a função recompensa, em oposição a função custo que foi utilizada em todos os demais MDPs apresentados até o momento. Essa mudança é devida a necessidade de apresentar a função de otimalidade em termos de maximização (em contraste a minimização, quando são utilizados custos) pois no Capítulo 9 será maximizada a probabilidade de custo-limite.

\subsection{Definição formal}

Um Processo de Decisão Markoviano Híbrido (HMDP), permite que o planejador atue sobre valores quantitativos de variáveis contínuas sem a necessidade de discretiza-las. Um exemplo da necessidade de adicionar raciocínio sobre variáveis continuas aparece quando queremos tratar a variável contínua (e.g. tempo) em intervalos não uniformes. Nesse exemplo, a discretização da variável contínua indisponibiliza a tomada de decisão em intervalos menores do que os valores definidos a priori.

Definição 15. Um Processo de Decisão Markoviano Híbrido (HMDP) é definido por uma tupla $\langle S, A, R, P\rangle$ :

- $S$ é o conjunto de estados, tal que $S=\vec{X} \times \vec{Y}$. O vetor $\vec{X}$ é um vetor de variáveis de estado booleanas $\left(X_{1}, \ldots, X_{n}\right)$ de comprimento $n$, uma atribuição de $\vec{X}$ é um vetor $\vec{x}=\left(x_{1}, \ldots, x_{n}\right)$ em que $x_{i} \in\{0,1\}$ é o valor da variável $X_{i}$. O vetor $\vec{Y}$ é um vetor de variáveis de estado continuas $\left(Y_{1}, \ldots, Y_{m}\right)$ de comprimento $m$, uma atribuição de $\vec{Y}$ é um vetor $\vec{y}=\left(y_{1}, \ldots, y_{m}\right)$ em que $y_{j} \in\left[L_{j}, U_{j}\right]$ e $L_{j} \leq U_{j} \in \mathbb{R}$. Portanto, um estado $s$ é uma atribuição das variáveis $s=(\vec{x}, \vec{y})=\left(x_{1}, \ldots, x_{n}, y_{1}, \ldots, y_{m}\right) \in \vec{X} \times \vec{Y} ;$

- $A$ é um conjunto finito de ações;

- $R$ é uma função de recompensa: $S \times A \rightarrow \mathbb{R}$, que atribui um valor a cada par estado-ação $(s, a)$;

- $P$ é a função de transição: $S \times A \rightarrow D(S)$, que atribui uma distribuição de probabilidades sobre o conjunto de estados $S$ a cada par estado-ação $(s, a)$. Denotamos $P\left(s^{\prime} \mid s, a\right)=$ $P\left(x_{1}^{\prime}, \ldots, x_{n}^{\prime}, y_{1}^{\prime}, \ldots, y_{m}^{\prime} \mid x_{1}, \ldots, x_{n}, y_{1}, \ldots, y_{m}, a\right)$ a densidade de probabilidade associada ao estado $s^{\prime}$ ao aplicar a ação $a$ no estado $s$; 
A Definição 15 é uma generalização da definição de um MDP fatorado com a adição de variáveis contínuas e funções parametrizadas por parâmetros contínuos. Tais adições acarretam na adição de uma importante problemática em HMDPs: Algumas funções de variáveis contínuas podem não permitir representações finitas exatas com um número finito de valores.

Devido a essa problemática apresentada, algoritmos de solução exata fazem suposições restritivas sobre as funções contínuas $R$ e $P$. Na Seção 6.2 tais suposições são discutidas juntamente com a apresentação do algoritmo de Programação Dinâmica Simbólica (SDP).

As transições de estados em HMDPs são definidas por Redes Bayesianas Dinâmicas (DBN) em que variáveis do próximo estado $x_{i}^{\prime}$ e $y_{i}^{\prime}$ são condicionadas em um subconjunto de variáveis de estado atual e próximo. Assim, a transição pode ser escrita como:

$$
P\left(\vec{x}^{\prime}, \vec{y}^{\prime} \mid \vec{x}, \vec{y}, a\right)=\prod_{i=1}^{n} P\left(x_{i}^{\prime} \mid \vec{x}, \vec{y}, a\right) \prod_{j=1}^{m} P\left(y_{j}^{\prime} \mid \vec{x}, \vec{x}^{\prime}, \vec{y}, a\right),
$$

em que $P\left(x_{i}^{\prime} \mid \vec{x}, \vec{y}, a\right)$ são funções de probabilidades condicionais (Conditional Probabilty Functions - CPFs) e $P\left(y_{j}^{\prime} \mid \vec{x}, \vec{x}^{\prime}, \vec{y}, a\right)$ é uma CPF definida pela função $\delta$ de Dirac. Neste modelo, as variáveis de próximo estado discretas $x_{i}^{\prime}$ são condicionadas por um subconjunto de variáveis de estado atual $\vec{x} \mathrm{e}$ $\vec{y}$. As variáveis de próximo estado contínuas $y_{j}^{\prime}$ são condicionadas por um subconjunto de variáveis de próximo estado discreta $\vec{x}^{\prime}$ e variáveis de estado atual $\vec{x}$ e $\vec{y}$.

Definição 16. Uma função $\delta$ de Dirac é uma distribuição na reta real que atribuí o valor 0 a todos os pontos exceto para o ponto zero em que o valor é infinito. Além disso, a integral da função $\delta$ de Dirac tem valor 1 . Podemos definir a função $\delta$ de Dirac em termos de outra função $f\left(x_{0}\right)$ como:

$$
\delta\left(x-f\left(x_{0}\right)\right)= \begin{cases}f\left(x_{0}\right)=x: & \infty \\ f\left(x_{0}\right) \neq x: & 0,\end{cases}
$$

sendo que:

$$
\int_{-\infty}^{\infty} \delta(x) d x=1 .
$$

Assim, a CPF $P\left(y_{j}^{\prime} \mid \vec{x}, \vec{x}^{\prime}, \vec{y}, a\right)$ é definida por:

$$
P\left(y_{j}^{\prime} \mid \vec{x}, \vec{x}^{\prime}, \vec{y}, a\right)=\delta\left(y_{j}^{\prime}-f\left(\vec{x}, \vec{x}^{\prime}, \vec{y}, a\right)\right),
$$

em que o valor de $y_{j}^{\prime}$ é definido deterministicamente (quando $y_{j}^{\prime}=f\left(\vec{x}, \vec{x}^{\prime}, \vec{y}, a\right)$ ).

No Capítulo 3 foi definido o objetivo de um GD-MDP como minimizar o custo acumulado esperado, foram definidas as funções para avaliar uma política (Equações 3.3 e 3.4) e como encontrar o valor ótimo de um GD-MDP seguindo tal critério. Posteriormente no Capítulo 4 foram estendidas tais definições para GD-MDPs fatorados. Finalmente, a seguir são estendidas essas equações para HMDPS.

A função de avaliação de uma política $\pi$ estacionária é dada pela recompensa acumulada esperada na execução de $\pi$ :

$$
f^{\pi}(\vec{x}, \vec{y})=E_{\pi}\left[\sum_{i=0}^{\infty} R\left(s^{i}, \pi\left(s^{i}\right)\right) \mid s^{0}=(\vec{x}, \vec{y})\right],
$$

em que $s^{i}$ representa o estado no $i$-ésimo estágio da execução da política $\pi$.

Pode-se expandir um passo dessa equação afim de explicitar a relação entre duas funções de avaliação sucessoras: 


$$
\begin{aligned}
& f^{\pi}(\vec{x}, \vec{y})=E_{\pi}\left[\sum_{i=0}^{\infty} R\left(s^{i}, \pi\left(s^{i}\right)\right) \mid s^{0}=(\vec{x}, \vec{y})\right] \\
& f^{\pi}(\vec{x}, \vec{y})=E_{\pi}\left[R\left(s^{0}, \pi\left(s^{0}\right)\right) \mid s^{0}=(\vec{x}, \vec{y})\right]+E_{\pi}\left[\sum_{i=1}^{\infty} R\left(s^{i}, \pi\left(s^{i}\right)\right) \mid s^{1}=\left(\vec{x}^{\prime}, \vec{y}^{\prime}\right) \sim P(\cdot \mid \vec{x}, \vec{y}, \pi(\vec{x}, \vec{y}))\right] \\
& f^{\pi}(\vec{x}, \vec{y})=R(\vec{x}, \vec{y}, \pi(\vec{x}, \vec{y}))+E_{\pi}\left[\sum_{i=1}^{\infty} R\left(s^{i}, \pi\left(s^{i}\right)\right) \mid s^{1}=\left(\vec{x}^{\prime}, \vec{y}^{\prime}\right) \sim P(\cdot \mid \vec{x}, \vec{y}, \pi(\vec{x}, \vec{y}))\right]
\end{aligned}
$$

O custo acumulado esperado a partir do estágio $i=1$ pode ser escrito em função da Equação 6.5, assim a Equação 6.6 pode ser escrita como:

$$
f^{\pi}(\vec{x}, \vec{y})=R(\vec{x}, \vec{y}, \pi(\vec{x}, \vec{y}))+E\left[f^{\pi}\left(s^{1}\right) \mid s^{1}=\left(\vec{x}^{\prime}, \vec{y}^{\prime}\right) \sim P(\cdot \mid \vec{x}, \vec{y}, \pi(\vec{x}, \vec{y}))\right]
$$

Finalmente, pode-se expandir o valor esperado apresentado na Equação 6.10 somando sobre todos os possíveis valores das variáveis de estado $\vec{x}^{\prime}$ e $\vec{y}^{\prime}$, e multiplicando a respectiva densidade de probabilidade de cada possível combinação:

$$
f^{\pi}(\vec{x}, \vec{y})=R(\vec{x}, \vec{y}, \pi(\vec{x}, \vec{y}))+\sum_{\vec{x}^{\prime} \in \vec{X}} \int_{\vec{y}^{\prime} \in \vec{Y}} f^{\pi}\left(\vec{x}^{\prime}, \vec{y}^{\prime}\right) * P\left(\vec{x}^{\prime}, \vec{y} \mid \vec{x}, \vec{y}, \pi(\vec{x}, \vec{y})\right) d \vec{y}^{\prime}
$$

A partir da Equação 6.10 é possível derivar a Equação de Otimalidade de Bellman para HMDP:

$$
f^{*}(\vec{x}, \vec{y})=\max _{a}\left[R(\vec{x}, \vec{y}, a)+\sum_{\vec{x}^{\prime} \in \vec{X}} \int_{\vec{y}^{\prime} \in \vec{Y}} f^{*}\left(\vec{x}^{\prime}, \vec{y}^{\prime}\right) * P\left(\vec{x}^{\prime}, \vec{y}^{\prime} \mid \vec{x}, \vec{y}, a\right) d \vec{y}^{\prime}\right]
$$

A seguir é mostrado como a Equação 6.11 é utilizada no Algoritmo de Programação Dinâmica Simbólica para encontrar soluções de HMDPs.

\subsection{Algoritmo de Programação Dinâmica Simbólica}

O Algoritmo de Programação Dinâmica Simbólica (SDP) [SDdB11] aplica o processo de iteração de valor utilizando representação simbólica similar ao algoritmo SPUDD apresentado no Capítulo 4. Porém, o algoritmo SDP usa XADDs no lugar de ADDs para permitir a representação eficiente de funções com variáveis contínuas.

Assim como feito para os outros algoritmos de iteração de valor pode-se descrever o algoritmo SDP matematicamente pelo processo de construção de uma série de funções $f^{i}$ iterativamente, para $i \geq 0$. Esse processo começa na inicialização de $f^{0}(\vec{x}, \vec{y})$ (por exemplo, $f^{0}(\vec{x}, \vec{y})=0$ ) o qual permite a construção das funções sucessoras $f^{i}(\vec{x}, \vec{y})$ seguindo a equação de atualização:

$$
f^{i+1}(\vec{x}, \vec{y})=\min _{a} Q^{i}(\vec{x}, \vec{y}, a),
$$

em que $Q^{i}(\vec{x}, \vec{y}, a)$ é definida como:

$$
Q^{i}(\vec{x}, \vec{y}, a)=R(\vec{x}, \vec{y}, a)+\sum_{\vec{x}^{\prime} \in \vec{X}} \int_{\vec{y}^{\prime} \in \vec{Y}} f^{i}\left(\vec{x}^{\prime}, \vec{y}^{\prime}\right) * P\left(\vec{x}^{\prime}, \vec{y}^{\prime} \mid \vec{x}, \vec{y}, a\right) d \vec{y}^{\prime} .
$$

O algoritmo termina esse processo de atualização quando $f^{i+1}=f^{i}$ e a política ótima é extraída por $\pi^{*}(\vec{x}, \vec{y})=\arg \min _{a} Q^{i}(\vec{x}, \vec{y}, a)$. 
No entanto como discutido anteriormente as funções contínuas necessitam assumir certas restrições para conseguir obter uma solução exata. Tais restrições são dadas pela representação das funções contínuas como funções baseadas em casos (descritas na Seção 2.2).

O Algoritmo 10, chamado de algoritmo de Programação Dinâmica Simbólica (Symbolic Dynamic Programming - SDP) para HMDPs é baseado no algoritmo SPUDD (descrito na Seção 4.2) para MDPs fatorados e pode ser divido em quatro etapas:

1. Marcação simbólica do próximo estado da função valor (Linha 7). Nessa etapa a função valor do horizonte anterior $f^{h-1}(\vec{x}, \vec{y})$ se torna a função dos estados sucessores, para isso é aplicada a operação de substituição com $\sigma=\left\{x_{1}=x_{1}^{\prime}, x_{2}=x_{2}^{\prime}, \ldots, x_{n}=x_{n}^{\prime}, y_{1}=y_{1}^{\prime}, y_{2}=y_{2}^{\prime}, \ldots, y_{m}=\right.$ $\left.y_{m}^{\prime}\right\}$, isto é $f^{h-1}\left(\vec{x}^{\prime}, \vec{y}^{\prime}\right)=f_{\sigma}^{h-1}(\vec{x}, \vec{y})$.

2. Integração sobre as variáveis contínuas (Linhas 8-10). Devido a independência entre as variáveis de próximo estado $y_{j}^{\prime}$ na definição da DBN, a marginalização das variáveis contínuas $y_{j}^{\prime}$ pode ser realizada em qualquer ordem. Assim, pode-se remover qualquer função que independa de $y_{j}^{\prime}$ e integrar apenas sobre as funções dependentes de $y_{j}^{\prime}$ (i.e., as funções $f^{h-1}\left(\vec{x}^{\prime}, \vec{y}^{\prime}\right)$ e $\left.P\left(y_{j}^{\prime} \mid \vec{x}, \vec{x}^{\prime}, \vec{y}, a\right)\right)$. A integração sobre $y_{j}^{\prime}$ é calculada por:

$$
\int_{y_{j}^{\prime}} f^{h-1}\left(\vec{x}^{\prime}, \vec{y}^{\prime}\right) * P\left(y_{j}^{\prime} \mid \vec{x}, \vec{x}^{\prime}, \vec{y}, a\right) d y_{j}^{\prime}=\int_{y_{j}^{\prime}} \delta\left(y_{j}^{\prime}-f\left(\vec{x}, \vec{x}^{\prime}, \vec{y}, a\right)\right) * f^{h-1}\left(\vec{x}^{\prime}, \vec{y}^{\prime}\right) d y_{j}^{\prime},
$$

como a função $\delta$ de Dirac define o valor de $y_{j}^{\prime}$ como $f\left(\vec{x}, \vec{x}^{\prime}, \vec{y}, a\right)$, que independe de $y_{j}^{\prime}$, pode-se aplicar a operação de substituição em $f^{h-1}\left(\vec{x}^{\prime}, \vec{y}^{\prime}\right)$ para $\sigma=\left\{y_{j}^{\prime}=f\left(\vec{x}, \vec{x}^{\prime}, \vec{y}, a\right)\right\}$. Assim, a integral pode ser reescrita como:

$$
f_{\sigma}^{h-1}\left(\vec{x}^{\prime}, \vec{y}^{\prime}\right) \int_{y_{j}^{\prime}} \delta\left(y_{j}^{\prime}-f\left(\vec{x}, \vec{x}^{\prime}, \vec{y}, a\right)\right) d y_{j}^{\prime}=f_{\sigma}^{h-1}\left(\vec{x}^{\prime}, \vec{y}^{\prime}\right)
$$

O cálculo da marginalização das variáveis contínuas pode ser realizado aplicando apenas a operação de substituição para todos as variáveis $y_{j}^{\prime}$. Como visto no exemplo no final da Seção 2.3, a substituição de uma função baseada em casos com parâmetro da função baseada em casos apresentada na integral é definida por:

$$
f_{\sigma}^{h-1}=\left\{\begin{array}{ll}
\sigma_{1}: & f_{y_{j}^{\prime}=f_{1}}^{h-1} \\
\sigma_{2}: & f_{y_{j}^{\prime}=f_{2}}^{h-1} \\
\vdots & \vdots \\
\sigma_{n}: & f_{y_{j}^{\prime}=f_{n}}^{h-1}
\end{array}= \begin{cases}\sigma_{1} \wedge \phi_{1}: & f_{y_{j}^{\prime}=f_{1}}^{1} \\
\sigma_{1} \wedge \phi_{2}: & f_{y_{j}^{\prime}=f_{1}}^{2} \\
\vdots & \vdots \\
\sigma_{1} \wedge \phi_{m}: & f_{y_{j}^{\prime}=f_{1}}^{m} \\
\vdots & \vdots \\
\sigma_{n} \wedge \phi_{1}: & f_{y_{j}^{\prime}=f_{n}}^{1} \\
\vdots & \vdots \\
\sigma_{n} \wedge \phi_{m}: & f_{y_{j}^{\prime}=f_{n}}^{m},\end{cases}\right.
$$

em que $\sigma_{i}$ e $f_{i}$ representam os casos e as respectivas funções definidas por cada caso para a função $f\left(\vec{x}, \vec{x}^{\prime}, \vec{y}, a\right)$, tal que $1 \leq i \leq n$; e:

$$
f_{y_{j}^{\prime}=f_{i}}^{h-1}= \begin{cases}\phi_{1}: & f_{y_{j}^{\prime}=f_{i}}^{1} \\ \phi_{2}: & f_{y_{j}^{\prime}=f_{i}}^{2} \\ \vdots & \vdots \\ \phi_{n}: & f_{y_{j}^{\prime}=f_{i}}^{m} .\end{cases}
$$


em que $\phi_{j}$ e $f^{\prime k}$ representam os casos e as respectivas funções definidas por cada caso para a função $f^{\prime h-1}$, tal que $1 \leq k \leq m$.

A marginalização contínua apresentada é realizada para cada ação $a$. O resultado de cada marginalização continua é denotado por $\tilde{Q}_{a}^{h+1}(\cdot)$.

3. Marginalização das variáveis discretas (Linhas 12-15). Essa etapa acontece de modo similar ao apresentado no algoritmo SPUDD. É aplicada a operação de sum-out apresentada para ADDs para cada variável $x_{i}^{\prime}$ em cada $\left[\tilde{Q}_{a}^{h+1}(\cdot) \otimes P\left(x_{i}^{\prime} \mid \vec{x}, \vec{y}, a\right)\right]$ para obter a marginalização discreta $Q_{a}^{h+1}(\cdot)$ para cada ação $a$.

4. Maximização sobre as ações (Linha 17). Finalmente, o valor ótimo é obtido aplicando a operação de maximização sobre cada ação $a$ e somando o valor da recompensa $R(\vec{x}, \vec{y}, a)$ :

$$
f^{h+1}=\max _{a}\left(Q_{a}^{h+1}(\cdot) \oplus R(\vec{x}, \vec{y}, a)\right) .
$$

Esse algoritmo é executado por $H$ passos, valor dado pelo usuário.

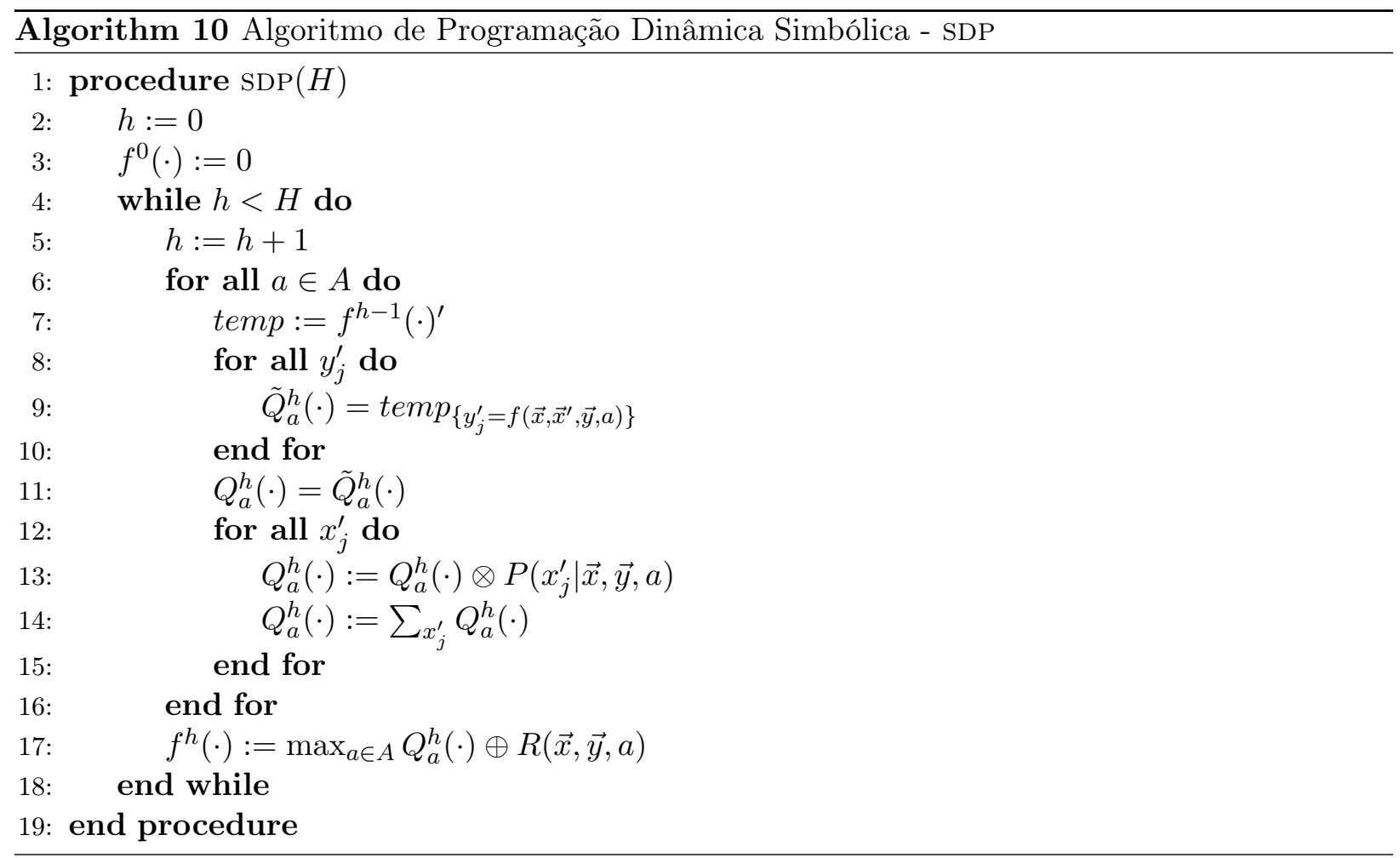




\section{Capítulo 7}

\section{Problema do Orçamento Mínimo}

A partir deste capítulo as propostas deste trabalho são apresentadas. Inicialmente é introduzida a definição formal do novo problema proposto no âmbito de RS-MDPs, chamado de Problema do Orçamento Mínimo. Este problema consiste em obter o orçamento mínimo para um RS-MDP para o qual as probabilidades de custo-limite não se alteram. Em sequência são abordadas as mudanças realizadas no algoritmo ITVI-DP para melhorar seu desempenho e para incluir a solução para este problema.

\subsection{Definição Formal}

Neste trabalho é proposto um novo problema, chamado de Problema do Orçamento Mínimo, no âmbito de RS-MDP: "Dado um RS-MDP, como encontrar o custo (orçamento) limite mínimo $\theta_{\text {min }}$ tal que $P^{*}\left(s, \theta_{\text {min }}\right)$ é máximo, $\forall s \in S$ ?".

Para o Problema do Orçamento Mínimo a convergência de um dado RS-MDP é definida quando as probabilidades de custo-limite para todos os estados $s$ atingem um máximo valor. Formalmente o critério de convergência é dado por:

Definição 17. Dado um RS-MDP, as probabilidades de custo-limite convergem em $\theta_{\min }$ quando $\exists \theta_{\min }<\infty$ tal que:

$$
P^{*}\left(s, \theta_{\text {min }}\right)=P^{*}\left(s, \theta_{b}\right), \forall \theta_{b}>\theta_{\text {min }}, \forall s \in S,
$$

em que $\theta_{\min }$ é o menor valor de orçamento que satisfaz essa equação.

Definição 18. O Problema do Orçamento Mínimo é definido como encontrar o valor de $\theta_{\min }$ que satisfaz a Definição 17.

Para um orçamento $\theta_{u}$ suficientemente grande, o algoritmo TVI-DP apresenta esse comportamento de convergência da probabilidade de custo-limite durante as iterações. A Tabela 7.1 mostra um exemplo de probabilidades custo-limite ótimas calculadas com o algoritmo TVI-DP para um RSMDP com 4 estados ( $s_{0}$ é o estado inicial; $s_{3}$ é o estado meta), $\theta_{u}=8$ and $c_{\max }=\max _{s, a}\{C(s, a)\}=$ 2. O algoritmo calcula a probabilidade de custo-limite ótima para $\theta$ variando entre 0 até $\theta_{u}$, com incremento de 1 . Note que para $\theta=2, P^{*}(s, \theta)$ é máximo $\forall s \in S$. Portanto, para esse exemplo $\theta_{\min }=2$

É possível garantir que a probabilidade de custo-limite ótima converge para $\theta$ quando $P^{*}(s, \theta)=$ $P^{*}\left(s, \theta-c_{\max }\right)$ para todo $s \in S$, em que $c_{\max }=\max _{s, a}\{C(s, a)\}$ é o maior custo, i.e., quando $\theta=\theta_{\min }+c_{\max }$ (Corolário 2). Para o exemplo, a probabilidade de custo-limite ótima converge em $\theta_{\min }=2$ e essa convergência pode ser identificada para $\theta=\theta_{\min }+c_{\max }=2+2=4$.

Para demonstrar o Corolário 2 são usados o Teorema 4 e Corolário 1.

Teorema 4. $P^{*}(s, \theta+i) \geq P^{*}(s, \theta), \forall s \in S, \forall i \geq 0$.

Demonstração. A probabilidade $P^{*}(s, \theta)$ é atualizada conforme a Equação 5.5, o que nos leva a dois possíveis cenários: 
Tabela 7.1: Probabilidades de custo-limite calculadas pelo algoritmo TVI-DP para cada estado aumentado $\left(s_{i}, \theta\right)$ considerando $\theta_{u}=8$

\begin{tabular}{c|l|c|c|c|}
\cline { 2 - 5 }$>4$ & 0.6 & 0.0 & 0.95 & 1.0 \\
\cline { 2 - 5 } 4 & 0.6 & 0.0 & 0.95 & 1.0 \\
\cline { 2 - 5 } 3 & 0.6 & 0.0 & 0.95 & 1.0 \\
\cline { 2 - 5 } 2 & 0.6 & 0.0 & 0.95 & 1.0 \\
\cline { 2 - 5 } 1 & 0.19 & 0.0 & 0.95 & 1.0 \\
\cline { 2 - 5 } 0 & 0.0 & 0.0 & 0.95 & 1.0 \\
\cline { 2 - 5 } \multicolumn{2}{c|}{$s_{0}$} & $s_{1}$ & $s_{2}$ & $s_{3}$
\end{tabular}

1. Se o orçamento corrente não é suficiente para nenhuma ação $a \in A, P^{*}(s, \theta)$ é zero; então para $\theta+1, P^{*}(s, \theta+1)$ pode ser: (i) zero, caso o orçamento $\theta+1$ ainda não permita ao agente aplicar nenhuma ação $a \in A$; (ii) um valor maior ou igual a zero, caso alguma ação $a$ requeira o custo $\theta+1$ (i.e. $\left.\sum_{s^{\prime} \in S} T\left(s, a, s^{\prime}\right) * P^{*}\left(s^{\prime}, \theta+1-C(s, a)\right) \geq 0\right)$. Note que para esse último caso $P^{*}(s, \theta+1)$ e maior que 0 , caso exista uma trajetória $h(s)$ partindo de $s$ e seguindo alguma política $\pi$ em que um estado meta $s_{g} \in S_{g}$ é atingido; ou $P^{*}(s, \theta+1)=0$, caso contrário.

2. Se o orçamento corrente é suficiente para alguma ação $a \in A, P^{*}(s, \theta) \geq 0$; então com $\theta+1$ a mesma ação ótima para o orçamento $\theta$ ainda pode ser aplicada, o que garante que a probabilidade $P^{*}(s, \theta+1)$ : (i) será igual a $P^{*}(s, \theta)$, caso a trajetória $h(s)$ para $\theta$ e a política $\pi$ se mantenham iguais para $\theta+1$ (Figura 7.1 (a) e (b)); (ii) será maior, caso uma ação melhor possa ser aplicada em algum estado $s_{h}$ pertencente a trajetória $h(s)$ para $\theta$ (i.e. alguma ação $a$ possuí custo igual ao orçamento restante $\theta_{h}+1$ e $\sum_{s^{\prime} \in S} T\left(s^{\prime} \mid s_{h}, a\right) * P^{*}\left(s^{\prime}, \theta_{h}+1-C\left(s_{h}, a\right)\right)>$ $\left.P^{*}\left(s_{h}, \theta_{h}\right)\right)$, resultando em uma nova trajetória $h^{\prime}(s)$ com maior probabilidade de alcançar um estado meta $s_{g} \in S_{g}$ e, portanto, $P^{*}(s, \theta+1)>P^{*}(s, \theta)$ (um exemplo desse caso é mostrado na Figura 7.1 (c)).

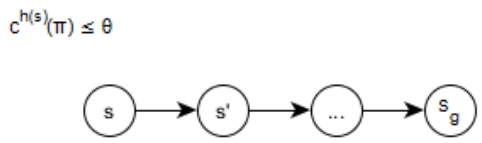

(a)

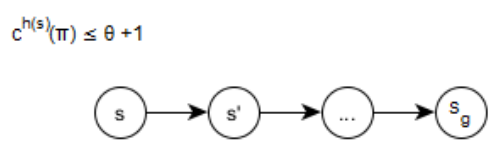

(b)

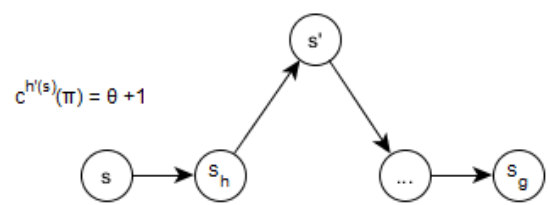

(c)

Figura 7.1: Exemplo usado na prova do Teorema 4. A figura mostra três possibilidades de históricos: (a) um histórico $h(\pi)$ com custo acumulado menor ou igual que $\theta$, em que $\pi$ é a política que maximiza a probabilidade de custo-limite $P^{\pi}(s, \theta)$; (b) o mesmo histórico $h(\pi)$ mostrado em (a), porém com custo acumulado menor ou igual que $\theta+1$, em que $\pi$ é a política que maximiza a probabilidade de custo-limite $P^{\pi}(s, \theta+1)$; e (c) um novo histórico com custo acumulado igual a $\theta+1$, em que $\pi$ é a política que maximiza a probabilidade de custo-limite $P^{\pi}(s, \theta+1)$.

Seja $c_{\max }$ o maior custo do RS-MDP, i.e., $c_{\max }=\max _{s, a}\{C(s, a)\}$.

Corolário 1. Se $P^{*}(s, \theta)=P^{*}\left(s, \theta-c_{\max }\right), \forall s \in S$, então $P^{*}(s, \theta)=P^{*}(s, \theta-i), \forall s \in S, \forall 0 \geq i \geq c_{\max }$.

Demonstração. Esse é o caso, pois as probabilidades de custo-limite são não-decrescentes conforme o orçamento $\theta$ aumenta, como demonstrado pelo Teorema 4 .

Corolário 2. As probabilidades de custo-limite são garantidas de convergir em $\theta$ quando: dado um $\theta, P^{*}(s, \theta)=P^{*}\left(s, \theta-c_{\max }\right), \forall s \in S$, i.e., quando $\theta=\theta_{\min }+c_{\max }$. 
Demonstração. Se $P^{*}(s, \theta)=P^{*}\left(s, \theta-c_{\max }\right)$ então $P^{*}(s, \theta+i)=P^{*}(s, \theta)$ para todo $i>0$. Isso pode ser provado por indução em $i$. Com $c_{\max }$, é garantido que todas as ações podem ser aplicadas na maximização. Portanto somente o terceiro caso da Equação 5.5 poderia mudar o valor de $P^{*}(s, \theta+i)$, mas o valor de $P^{*}\left(s^{\prime}, \theta+i-C(s, a)\right)$ no lado direito da terceira atribuição na Equação 5.5 pode sempre ser escrito como $P^{*}\left(s^{\prime}, \theta\right)$, porque $\theta+i-C(s, a)$ estará no intervalo $\left[\theta-c_{\max }+1, \theta+i-1\right]^{1}$. Portanto, $P^{*}(s, \theta+i)=P^{*}(s, \theta)$ para todo $i>0$.

\subsection{Iteração de Valor Topológico Melhorado (ITVI-DP)}

Duas melhorias para o algoritmo TVI-DP foram implementadas: (i) uma checagem de convergência das probabilidades de custo-limite ótimas (que é também utilizada como uma condição adicional de parada para o algoritmo); e (ii) a geração de estados aumentados que pertencem a uma história que termina em um estado meta.

Assim como o algoritmo TVI-DP, ITVI-DP (Algoritmo 11) primeiro inicializa as probabilidades de custo-limite para os estados metas em 1 (Linha 2 ) e $P^{*}(s, 0)$ com 0 , para todos $s \notin S_{g}$ (Linha 3). Em seguida, uma busca em profundidade partindo dos estados metas é realizada para encontrar o conjunto de SCCs $Y$ (Linha 4). Isso é realizado executando o algoritmo de Tarjan [Tar71] no grafo transposto, em que todas as transições são invertidas (i.e., existe um hiper-arco $\left(s^{\prime}, a, s\right)$, se e somente se $T\left(s, a, s^{\prime}\right)>0$ ). No entanto, ITVI-DP melhora a geração de estados aumentados do algoritmo TVI-DP, gerando-os durante o algoritmo de Tarjan. Assim, somente estados aumentados pertencentes a uma história que termina em um estado meta são gerados, diminuindo o espaço de estados explicitamente declarados.

Diferentemente do algoritmo TVI-DP, ITVI-DP computa $c_{\max }$ na Linha 5 , que é usado na checagem de convergência. O processo a seguir (Linhas 6-12) é repetido para orçamentos $\theta$ começando em 0 até $\theta_{u}$ :

- ITVI-DP chama o método CONVERGENCECHECK na Linha 7 para checar a convergência. A checagem de convergência para um dado orçamento e $c_{\max }$ segue o Corolário 2 (ver Algoritmo 12). Se a convergência é alcançada o algoritmo ITVI-DP para e retorna $c_{\min }$.

- Para cada SCC $Y^{\theta}$ em que estados aumentados possuem o mesmo orçamento $\theta$, ITVI-DP atualiza cada SCC $Y^{\theta} \in Y$ em ordem topológica reversa usando uma modificação do algoritmo RSVI, chamada MRSVI (Linhas 9-11).

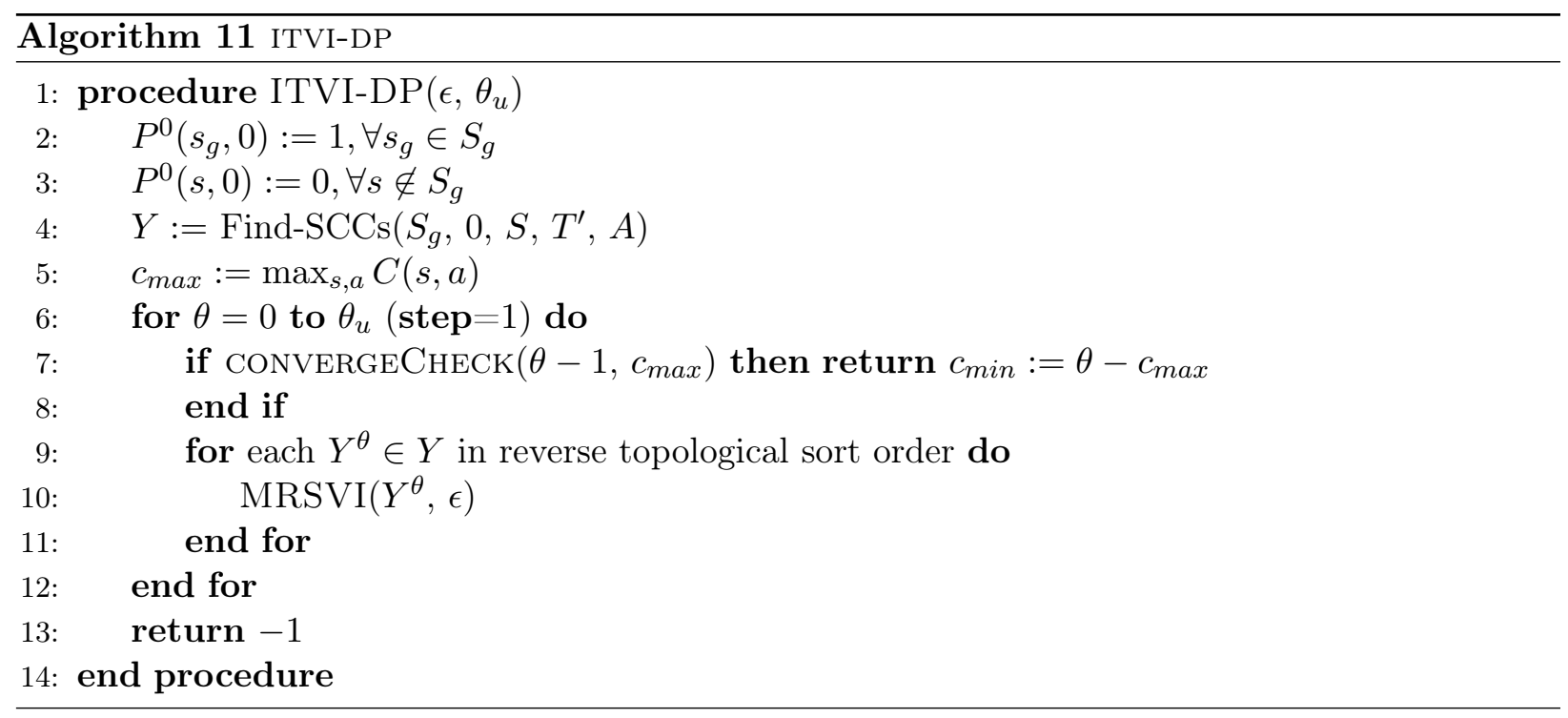

${ }^{1}$ É considerado que i é um inteiro sem perda de generalidade. 


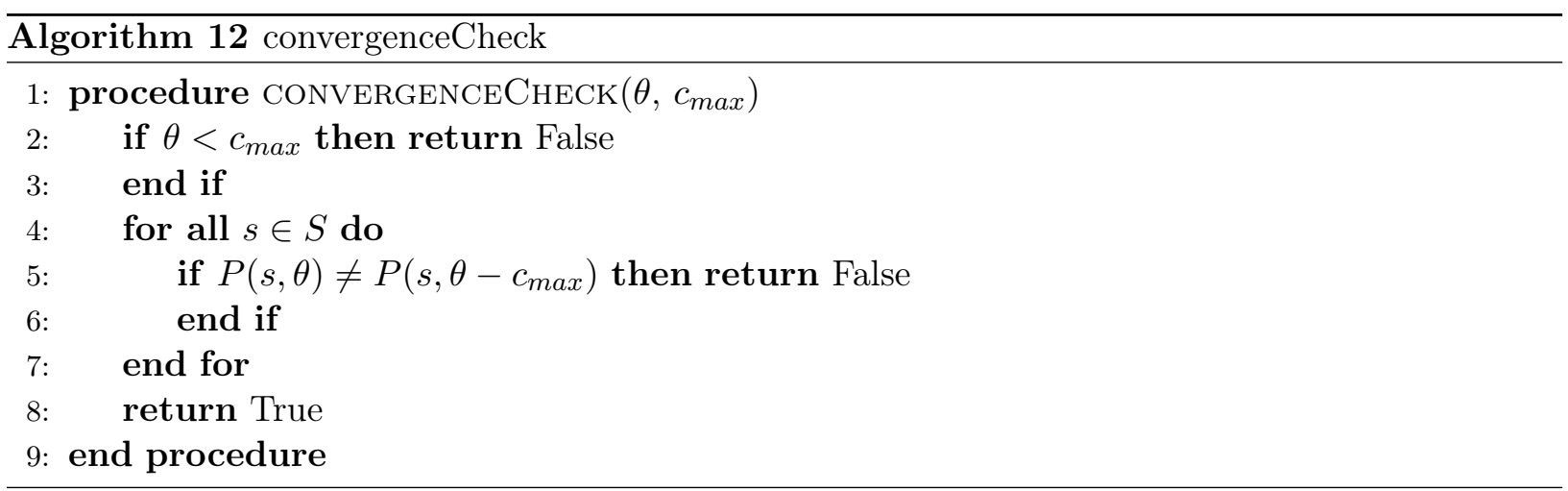

O algoritmo MRSvi (Algoritmo 13) chama o algoritmo searchP (Algoritmo 19) para calcular $P^{i+1}(s, \theta)$ na Linha 7 e para calcular uma política $\pi$ na Linha 13. O Algoritmo searchP procura pelo estado aumentado mais próximo $\left(s^{\prime}, \theta^{\prime}\right)$ em ordem decrescente de $\theta^{\prime}$ tal que $\theta^{\prime}<\theta-C(s, a)$, que corresponde a um estado aumentado em memória. Note que ITVI-DP não perde nenhum valor de $P\left(s^{\prime}, \theta^{\prime}\right)$.

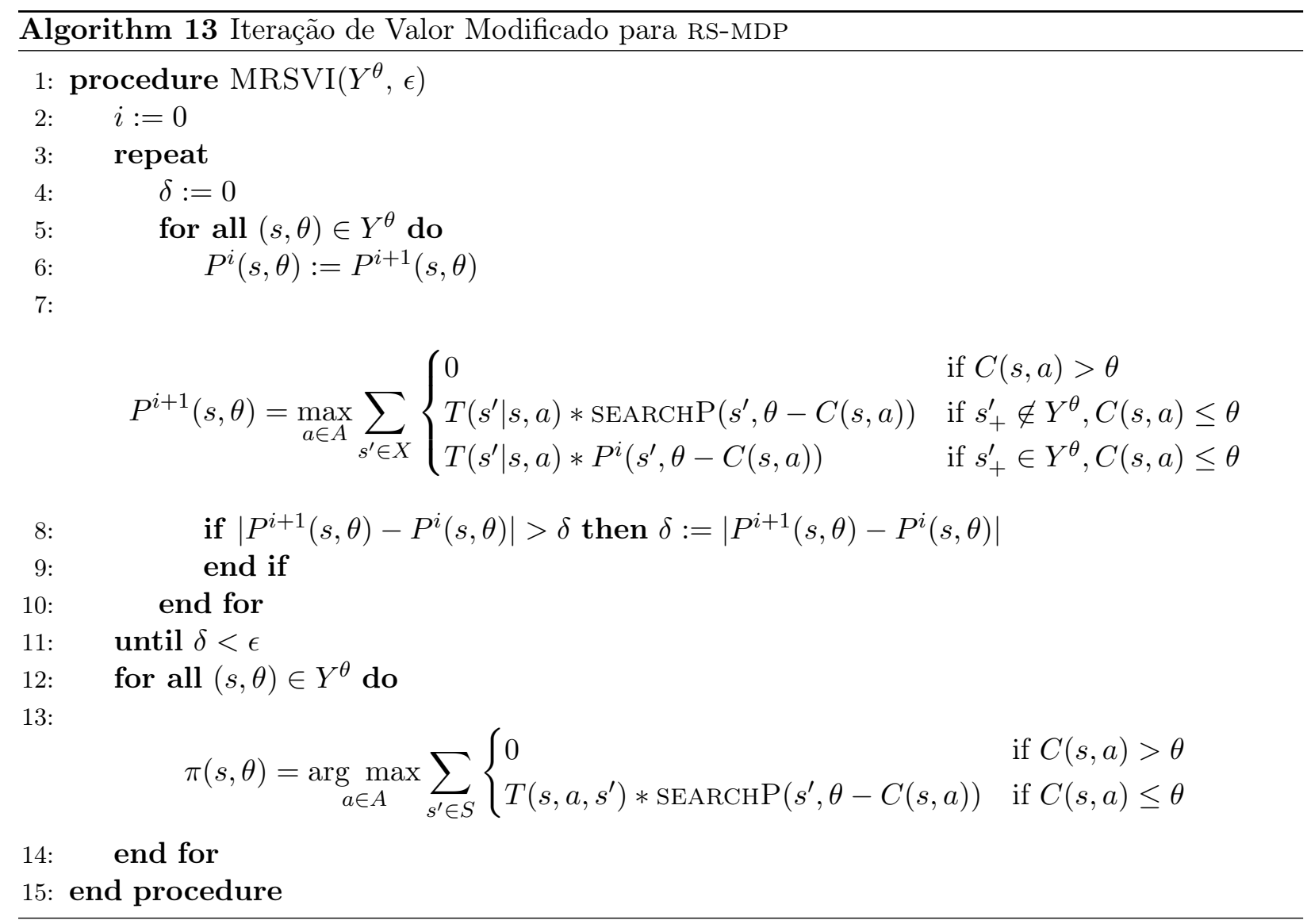

A Figura 7.2 ilustra o grafo gerado pelo algoritmo ITVI-DP para o exemplo dado na Fig. 5.1. Note que ITVI-DP gera um número menor de estados aumentados que TVI-DP já que ITVI-DP gera somente estados aumentados que alcançam um estado aumentado meta, isso é $\left(s_{3}, 0\right)$, para esse exemplo. Após encontrar as SCCs, na primeira iteração das Linhas 6-12 do Algoritmo 11 (quando $\theta=0$ ), ITVI-DP realiza a checagem de convergência e atualiza cada SCC com $\theta=0$ em ordem topológica reversa, i.e., atualiza a primeira SCC a qual tem o estado $\left(s_{3}, 0\right)$ e então atualiza a segunda SCC que tem o estado $\left(s_{2}, 0\right)$. A primeira linha da Tabela 7.1 mostra as probabilidades de custo-limite ótimas obtidas após realizar essas atualizações. Na segunda iteração das Linhas 6-12 (em que $\theta=1$ ), ITVI-DP checa novamente a convergência e atualiza cada SCC $\operatorname{com} \theta=1$, i.e. o terceiro SCC que tem o estado $\left(s_{0}, 1\right)$ e o quarto SCC que tem o estado $\left(s_{2}, 1\right)$. 


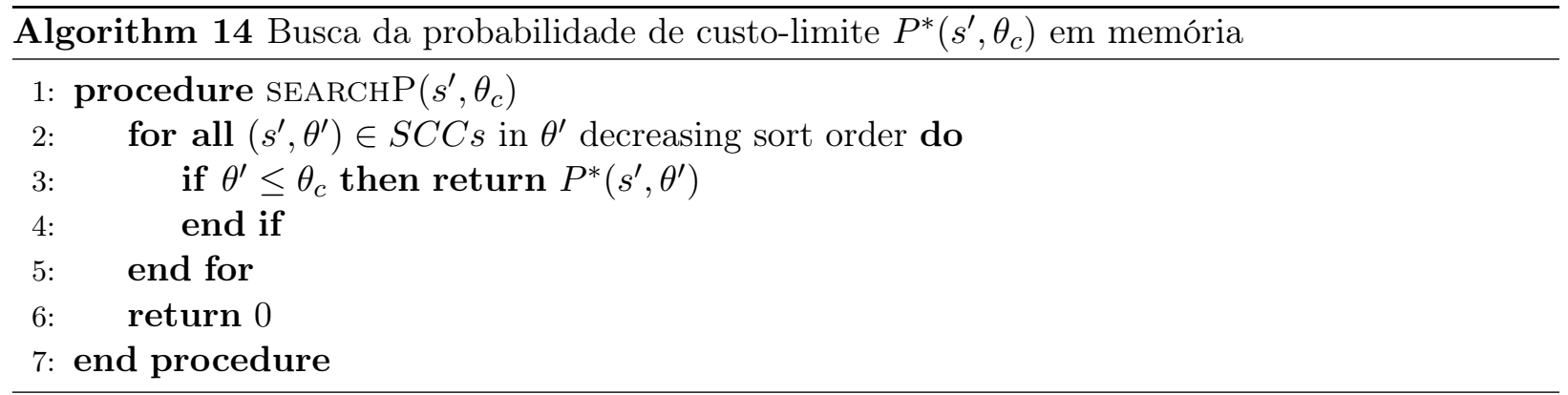

A Tabela 7.1 mostra as probabilidades de custo-limite ao executar o algoritmo ITVI-DP para o exemplo dado. Note que para qualquer $\theta>2$ nenhuma probabilidade é alterada, porém como $c_{\max }=2$ somente para $\theta=4$ que o algoritmo ITVI-DP pode identificar que as probabilidades de custo-limite convergiram. Portanto, para qualquer valor de $\theta>4$ ITVI-DP não precisa calcular nenhum valor adicional, diferentemente do algoritmo TVI-DP.

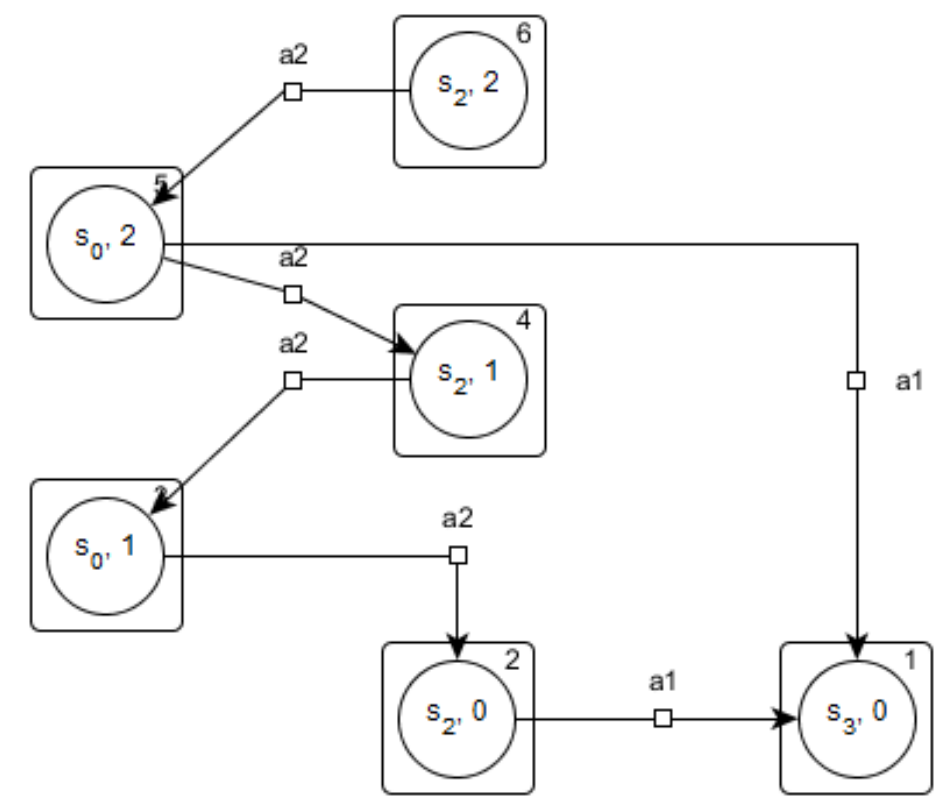

Figura 7.2: Grafo das SCCs para os estados aumentados gerado pelo algoritmo ITVI-DP para o RS-MDP da Figura 5.1.

\subsection{Espaço de Estados Aumentados}

Os algoritmos TVI-DP e ITVI-DP variam o orçamento disponível de 0 até $\theta_{u}$, incrementando $\theta$ em 1 (veja Linha 5 do Algoritmo 9 e Linha 6 do Algoritmo 11). Essa escolha de incremento pode causar diversos cálculos desnecessários das probabilidades de custo-limite quando o aumento de orçamento disponível entre duas iterações subsequentes não é o suficiente para modificar a probabilidade de custo-limite. Portanto, é proposto um método, baseado no problema de satisfação com restrição (constrained satisfaction problem - CSP), para encontrar o conjunto $\Theta_{r}$ de todos os orçamentos válidos de 0 até $\theta_{u}$ de um RS-MDP, dado pela solução do seguinte CSP:

$$
d_{1} * c_{1}+d_{2} * c_{2}+\ldots+d_{m} * c_{m} \leq \theta_{u},
$$

em que $c_{i}, 1 \leq i \leq m$, são todos os elementos do contradomínio da função de custo de um RS-MDP; e $d_{i} \in \mathbb{N}$, representa a quantidade de vezes que as ações com custo $c_{i}$ são aplicadas. Então, essa 
expressão representa todas as possíveis combinações de orçamento restante, dado um RS-MDP.

A seguir é demonstrado que o conjunto de todos os orçamentos válidos $\Theta_{r}$ é composto por todas as soluções da Inequação $7.2 \mathrm{com}$ valores variando de $d_{i} \in \mathbb{N}$. O Teorema 5 afirma que para cada orçamento possível calculado: (1) o orçamento está no conjunto $\Theta_{r}$ e portanto a probabilidade de custo-limite para esse orçamento precisa ser calculada; ou (2) o orçamento não está no conjunto $\Theta_{r}$ e portanto a probabilidade de custo-limite para esse orçamento é irrelevante já que deve existir uma probabilidade de custo-limite de mesmo valor, porém para um orçamento menor em $\Theta_{r}$.

Teorema 5. Dado um RS-MDP, e $\Theta_{r}$ com todas as soluções para a Inequação 7.2 , em ordem crescente, $\forall \theta_{y} \notin \Theta_{r}$ em que $\theta_{y}<\theta_{u}$, existe $\theta_{x}=\sup \left\{\theta_{i} \in \Theta_{r} \mid \theta_{i}<\theta_{y}\right\}$ tal que $P\left(s, \theta_{y}\right)=P\left(s, \theta_{x}\right)$, $\forall s \in S$.

Demonstração. Seja $\operatorname{Tr}$ o conjunto de todas as possíveis histórias de um RS-MDP, assuma que exista um $\theta_{y} \notin \Theta_{r}$ tal que $P\left(s, \theta_{y}\right) \neq P\left(s, \theta_{x}\right)$; então deve existir uma nova política $\pi$ que pode ser aplicada e $\theta_{y}=\sup \left\{\sum_{s_{i} \in h} C\left(s_{i}, \pi\left(s_{i}\right)\right) \mid h \in T r\right\}$. Isto é, se existe um orçamento $\theta_{y}$ para o qual não existe nenhum orçamento menor com probabilidade de custo-limite igual a $P\left(s, \theta_{y}\right)$ então tal orçamento $\theta_{y}$ permite a substituição de uma ação em $\pi$, tal que uma das trajetórias considerando essa nova política maximiza $P\left(s, \theta_{y}\right)$ e resulta em custo acumulado igual a $\theta_{y}$. Isto implica que $\theta_{y}=\sum_{s_{i} \in t_{\text {sup }}} C\left(s_{i}, \pi\left(s_{i}\right)\right)=C\left(s_{0}, \pi\left(s_{0}\right)\right)+C\left(s_{1}, \pi\left(s_{1}\right)\right)+\ldots+C\left(s_{l}, \pi\left(s_{l}\right)\right)=c_{1}+c_{2}+\ldots+c_{l} \notin \Theta_{r}$. Isso é uma contradição já que por definição $c_{1}+c_{2}+\ldots+c_{l}$ é um elemento de $\Theta_{r}$. 


\section{Capítulo 8}

\section{Processos de Decisão Markovianos Sensíveis ao Risco Fatorados}

Neste capítulo é introduzida a definição de Processos de Decisão Markovianos Sensíveis ao Risco Fatorado. Neste modelo estados são representados pela combinação de variáveis booleanas e um orçamento limite. É proposta também uma solução para este modelo baseado no algoritmo SPUDD.

\subsection{Definição Formal}

Um RS-MDP fatorado é definido por uma tupla $\left\langle M_{f}, \theta_{u}\right\rangle$ :

- $M_{f}$ é um MDP Fatorado, como definido anteriormente;

- $\theta_{u} \in \mathbf{R}^{+}$é o custo (ou orçamento) limite definido pelo usuário.

O conjunto $\boldsymbol{\Theta}$ denota todos os possíveis custos limites, isto é, $\boldsymbol{\Theta}=\left\{0,1, \ldots, \theta_{u}\right\}$. Em RS-MDPs fatorados estados aumentados são representados por um par $s=(\vec{x}, \theta)$, em que $\vec{x} \in\{0,1\}^{n}$ e $\theta \in \Theta$.

A função de otimilidade para RS-MDPs enumerativos (Equação 5.5) pode ser reescrita como:

$$
p^{*}(\vec{x}, \theta)=\max _{a \in A} \sum_{\vec{x}^{\prime} \in S} \begin{cases}0 & \text { se } C_{f}(\vec{x}, a)>\theta, \\ 1 & \text { se } C_{f}(\vec{x}, a) \leq \theta \text { e } \vec{x} \in G, \\ \operatorname{Pr}\left(\vec{x}^{\prime} \mid a, \vec{x}\right) p^{*}\left(\vec{x}^{\prime}, \theta-C_{f}(\vec{x}, a)\right) & \text { se } C_{f}(\vec{x}, a) \leq \theta \text { e } \vec{x} \notin G .\end{cases}
$$

A Equação 8.1 pode ser calculada eficientemente usando ADDs para representar as probabilidades de custo-limite $p^{*}(\vec{x}, \theta)$, as probabilidades condicionais $\operatorname{Pr}\left(X_{i}^{\prime} \mid \operatorname{pa}\left(X_{i}^{\prime}\right), a\right)$ e a função de custo $C_{f}(\vec{x}, a)$.

\subsection{Algoritmo RS-SPUDD}

O algoritmo RS-SPUDD utiliza ADDs para representar: (i) a função de custo para cada ação $a$, denotada $C_{D D}(\cdot, a)$; (ii) as probabilidades de custo limite de cada iteração $i$ para cada valor de $\theta$, denotado por $p_{D D}^{i}(\cdot, \theta)$, i.e., é mantido um $p^{i}$ separado para cada $\theta$; e (iii) a função de transição para cada par $\left(X_{i}, a\right)$, denotado por $\operatorname{Pr}_{D D}$. O Algoritmo RS-SPUDD atualiza todos os estados iterativamente aplicando o seguinte conjunto de equações, que representam a Equação 5.7 em forma fatorada:

$$
\begin{gathered}
p_{D D}^{i+1}(\vec{x}, \theta)=\max _{a \in A} Q_{D D}^{i}(\vec{x}, a, \theta) \\
Q_{D D}^{i}(\vec{x}, a, \theta)=\sum_{\overrightarrow{x^{\prime}}} \bigotimes_{j=1}^{n} \operatorname{Pr}_{D D}\left(X_{j}^{\prime} \mid p a\left(X_{j}^{\prime}\right), a\right) \otimes W_{D D}^{i}(\vec{x}, a, \theta)
\end{gathered}
$$


em que $W_{D D}^{i}(\vec{x}, a, \theta)$ é:

$$
W_{D D}^{i}(\vec{x}, a, \theta)= \begin{cases}0 & \text { se } C_{D D}(\vec{x}, a)>\theta \\ p_{D D}^{*}\left(\overrightarrow{x^{\prime}}, \theta-C_{D D}(\vec{x}, a)\right) & \text { se } 0<C_{D D}(\vec{x}, a) \leq \theta, \overrightarrow{x^{\prime}} \notin G \\ p_{D D}^{i}\left(\overrightarrow{x^{\prime}}, \theta\right) & \text { se } C_{D D}(\vec{x}, a)=0, \overrightarrow{x^{\prime}} \notin G \\ 1 & \text { se } C_{D D}(\vec{x}, a) \leq \theta, \overrightarrow{x^{\prime}} \in G,\end{cases}
$$

em que $i$ representa a i-ésima iteração e $p_{D D}^{0}(\cdot, \theta)$ é inicializado com a função indicadora $G_{o a l} l_{D D}$ que atribui o valor 1 para os estados meta e 0 para os demais estados, i.e.:

$$
\operatorname{Goal}_{D D}(\vec{x})= \begin{cases}1 & \text { se } \vec{x} \in G \\ 0 & \text { caso contrário. }\end{cases}
$$

Uma vez que o algoritmo RS-SPUDD calcula $p^{i}$ em ordem crescente dos orçamentos $\theta, p_{D D}^{*}\left(\vec{x}^{\prime}, \theta-\right.$ $\left.C_{D D}(\vec{x}, a)\right)$ já foi computado (segundo caso da Equação 8.4) pois $\theta-C_{D D}(\vec{x}, a)<\theta$. No terceiro caso (quando o custo imediato é igual a zero) é necessário usar $p^{i}$ para calcular $p^{i+1}$.

As operações em 8.2, 8.3 e 8.4 podem ser calculadas através de operações em diagramas de decisão. Porém, a principal dificuldade em computar a Equação 8.4 com operações entre ADDs é derivado do segundo caso: a função de custo $C_{D D}(\vec{x}, a)$ do estado corrente e ação é um parâmetro da probabilidade de custo-limite ótima para o próximo estado $\vec{x}^{\prime}$. Então, o cálculo de $p_{D D}^{*}\left(\vec{x}^{\prime}, \theta-\right.$ $\left.C_{D D}(\vec{x}, a)\right)$ depende de vários $p_{D D}^{*}\left(\cdot, \theta^{\prime}\right)$, um para cada valor $\theta^{\prime}=\theta-C_{D D}(\vec{x}, a)$ que foi calculado anteriormente. Portanto, para realizar o cálculo da Equação 8.3, o algoritmo RS-SPUDD junta todas as probabilidades de custo-limite de estados sucessores em um único ADD, chamado de $W_{D D}^{i}$.

O Algoritmo 15 calcula a Equação 8.4. Dado $p_{D D}^{*}(\cdot, \cdot)$ previamente computados; o orçamento corrente $\theta ; p_{D D}^{i}(\cdot, \theta)$ para o orçamento corrente $\theta$; e a função de custo para uma ação especifica $a$ $\left(C_{D D}(\cdot, a)\right)$, o algoritmo calcula $W_{D D}^{i}\left(\vec{x}, a, \theta, \vec{x}^{\prime}\right)$. Primeiro, GETW constrói um conjunto de funções indicadoras chamado de $I$ (Algoritmo 15, Linha 2), uma função indicadora $A_{D D}^{c, a}$ para cada ação $a$ e para cada diferente custo $c$ de $C_{f}(\cdot, a)$ que não é maior que o orçamento corrente $\theta$. Essa função indicadora recebe o valor de 1 para estados com custo $c$ e 0 caso contrário, i.e.:

$$
A_{D D}^{c, a}(\vec{x})= \begin{cases}1 & \text { se } C_{D D}(\vec{x}, a)=c \\ 0 & \text { se } C_{D D}(\vec{x}, a) \neq c .\end{cases}
$$

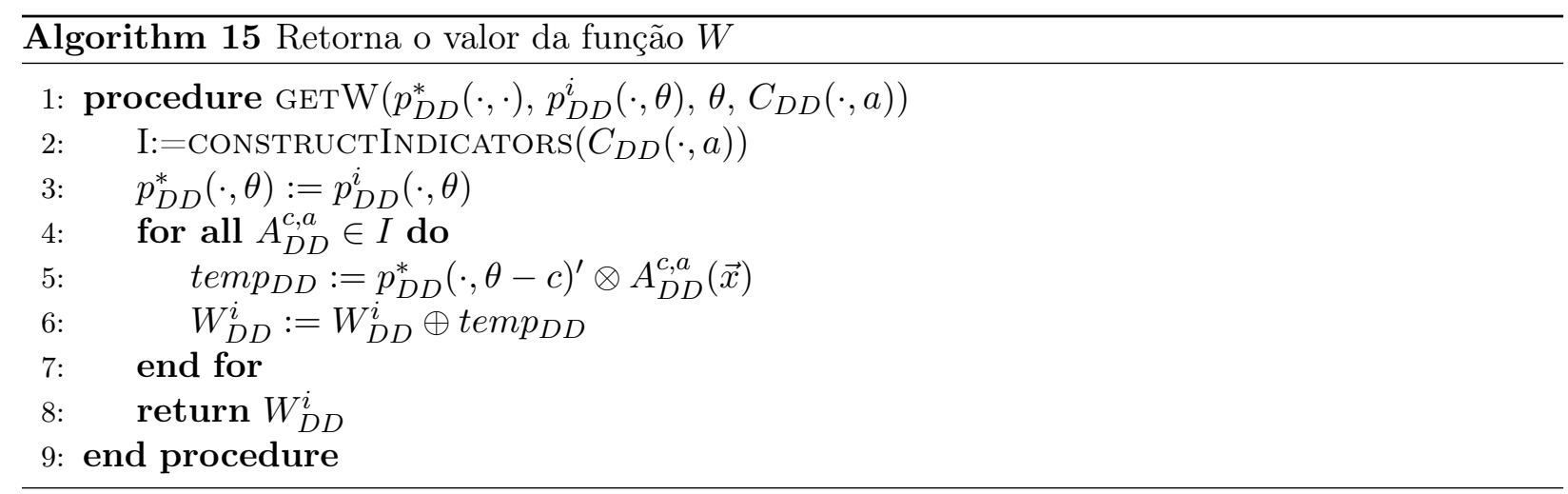

Em seguida, na Linha $3 \mathrm{GETW}$ atribui $p_{D D}^{i}(\cdot, \theta)$ para $p_{D D}^{*}(\cdot, \theta)$ (o que corresponde ao terceiro caso da Equação 8.4) e calcula um único ADD $W_{D D}^{i}\left(\vec{x}, a, \theta, \vec{x}^{\prime}\right)$ realizando as seguintes operações:

$$
W_{D D}^{i}\left(\vec{x}, a, \theta, \vec{x}^{\prime}\right)=\sum_{A_{D D}^{c, a} \in I} p_{D D}^{*}(\vec{x}, \theta-c)^{\prime} \otimes A_{D D}^{c, a}(\vec{x}),
$$

em que $p_{D D}^{*}(\cdot, \theta)^{\prime}$ é o $\operatorname{ADD} p_{D D}^{*}(\cdot, \theta)$ com todas as variáveis seguidas de apóstrofe para representar probabilidades de custo-limite ótima do próximo estado. 
A Figura 8.1 ilustra um exemplo do cálculo de $W_{D D}^{i}$ para $\theta=25, i=0$ e $a=a_{1}$. Primeiro, considerando que a ação $a_{1}$ possuí 3 diferentes custos $(5,10$ e 0), o algoritmo cria três funções indicadoras $A_{D D}^{5, a_{1}}, A_{D D}^{10, a_{1}}$ e $A_{D D}^{0, a_{1}}$, que são multiplicadas respectivamente pelas probabilidades de custo-limite $p_{D D}^{*}(\cdot, \theta-5)^{\prime}, p_{D D}^{*}(\cdot, \theta-10)^{\prime}$ e $p_{D D}^{*}(\cdot, \theta-0)^{\prime}$. Note que $p_{D D}^{*}(\cdot, \theta-0=25)^{\prime}$ é igual a $P_{D D}^{0}(\cdot, \theta-0=25)^{\prime}$ (terceiro caso da Equação 8.4 ). Finalmente, GETW calcula a soma entre as funções obtidas gerando a função $W_{D D}^{0}\left(\cdot, a_{1}, 25, \cdot\right)$.
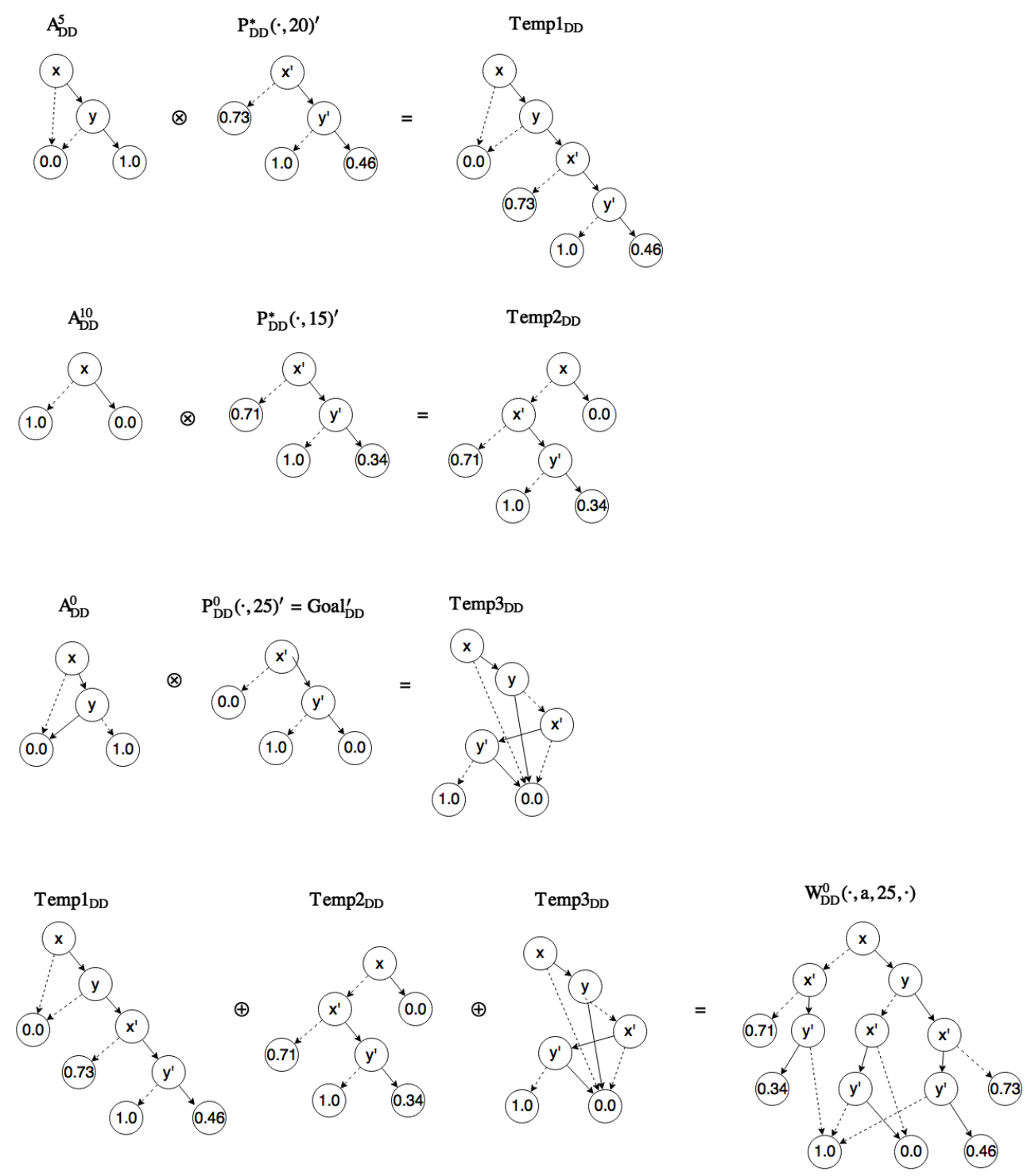

Figura 8.1: Exemplo do cálculo de $W_{D D}^{0}\left(\cdot, a_{1}, 25, \cdot\right)$.

Após obter a função $W_{D D}^{i}(\cdot, a, \theta, \cdot)$, é possível calcular a função $Q_{D D}^{i}(\cdot, a, \theta)$ (Equação 8.3) eficientemente eliminando cada variável $X_{i}^{\prime}$ (aplicando a operação de marginalização sobre as variáveis nos ADDs) sempre que essa variável não dependa de nenhuma outra. Por exemplo, se $X_{1}^{\prime}$ não depende de nenhuma outra variável $X_{j}^{\prime}, \forall j \neq 1$ usando a ação $a$, é possível colocar para fora a soma sobre $X_{1}^{\prime}$ expandindo a Equação 8.3 em: 


$$
\begin{aligned}
& Q_{D D}^{i}(\cdot, a, \theta)=\sum_{X_{2}^{\prime}, \ldots, X_{n}^{\prime}} \bigotimes_{j=2}^{n} \operatorname{Pr}_{D D}\left(x_{j}^{\prime} \mid p a_{a}\left(X_{j}^{\prime}\right), a\right) \otimes \\
& \sum_{X_{1}^{\prime}} \operatorname{Pr}_{D D}\left(X_{1}^{\prime} \mid p a\left(X_{1}^{\prime}\right), a\right) W_{D D}^{i}(\cdot, a, \theta, \cdot)
\end{aligned}
$$

o que pode ser repetido para todas as demais variáveis restantes resultando no $\operatorname{ADD} Q_{D D}^{i}(\cdot, a, \theta)$.

A Figura 8.2 mostra a multiplicação de $W_{D D}^{i}\left(\cdot, a_{1}, 5, \cdot\right)$ por $\operatorname{Pr}_{D D}\left(X^{\prime} \mid p a\left(X^{\prime}\right), a_{1}\right)$ que resulta em um diagrama temporário $T e m p 1_{D D}$. A eliminação da variável $x^{\prime}$ é realizada usando a operação de marginalização em ADDs (também chamada de sum-out) sobre esse diagrama temporário resultando em um novo diagrama temporário Temp $2_{D D}$. A operação de eliminação de variável é repetida para as variáveis de próximo estado restantes ( $y^{\prime}$ no exemplo).
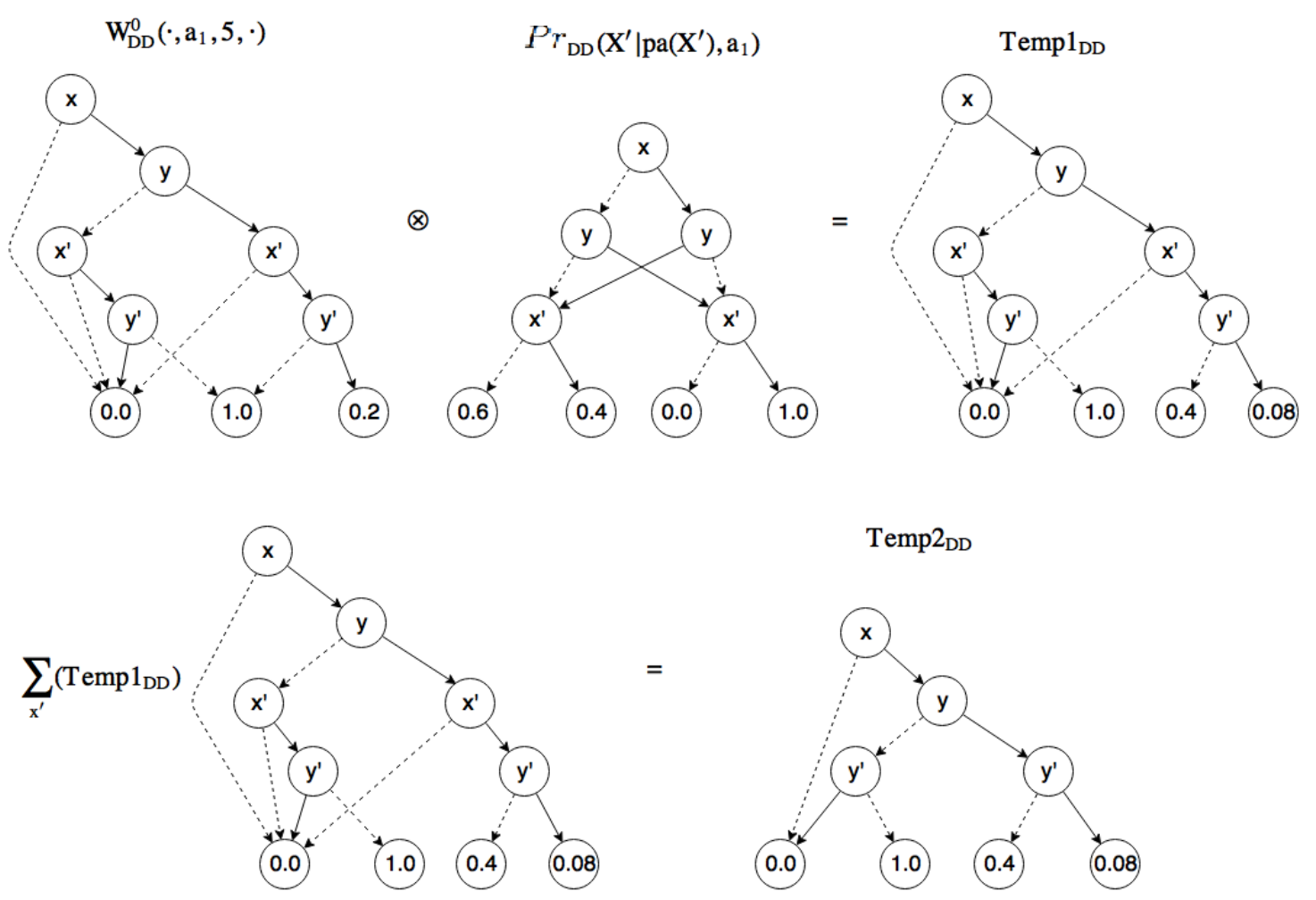

Figura 8.2: Exemplo da marginalização sobre a variável $x^{\prime}$.

Assim que todas as funções $Q_{D D}^{i}(\cdot, a, \theta)$ para cada ação $a$ são obtidas, o algoritmo calcula a probabilidade $p_{D D}^{i+1}(\cdot, \theta)$ aplicando o operador de maximização de ADDs sobre todas as funções $Q_{D D}^{i}(\cdot, a, \theta)$ (Equação 8.2).

\section{Pseudocódigo do RS-SPUDD}

O Algoritmo RS-SPUDD (Algoritmo 16) calcula a Equação 8.2 como descrita anteriormente e inclui duas melhorias: (i) uma checagem de convergência; e (ii) o cálculo de orçamentos válidos.

A checagem de convergência (Corolário 2) afirma que é possível garantir que as probabilidades de custo-limite convergem para $\theta$ se $p^{*}(s, \theta)=p^{*}\left(s, \theta-c_{\max }\right)$ para todo $s \in S$. Então, o algoritmo RS-SPUDD chama primeiro o método GETMAXVALUE para encontrar o maior custo $c_{\max }$ (Linha 2 ). Esse valor é usado pelo algoritmo CONVERGENCECHECK que checa a convergência e é chamado na Linha 5 do algoritmo RS-SPUDD. Note que a checagem de convergência somente precisa comparar dois ADDs, o que pode ser feito eficientemente utilizando códigos de hash.

Para calcular o conjunto de orçamentos válidos $\Theta_{r}$, o algoritmo chama o resolvedor de CSP 
(SOLVECSP) na Linha 3. Nas Linhas 4-23, o algoritmo RS-SPUDD calcula $p^{*}$ em ordem crescente dos orçamentos $\theta \in \Theta_{r}$.

Na Linha $8, p_{D D}^{0}(\cdot, \theta)$ é inicializado com a função indicadora $\operatorname{Goal}_{D D}$ que atribui o valor 1 se o estado é meta e 0 caso contrário.

Em cada iteração $i, p_{D D}^{i}(\cdot, \theta)$ é calculada (Linhas 9-21) até que o máximo residual seja menor que $\epsilon$. O algoritmo calcula $W_{D D}^{i}$ na Linha 12 . Já que alguns $p_{D D}^{*}$ podem não ter sido calculados quando consideramos somente o conjunto $\Theta_{r}$, o método GETW (Algoritmo 15) precisa ser modificado. Esse método modificado é nomeado de MGeTW e chamado na Linha 12 do algoritmo RS-SPUDD. A eliminação de variáveis é realizada nas Linhas $14-17$. Uma vez obtidas $Q_{D D}^{i}(\cdot, a, \theta)$ para todas as ações, o algoritmo calcula $p_{D D}^{i}(\cdot, \theta)$ na Linha 20. Finalmente, na Linha 24 , o valor de $\theta$ encontrado é devolvido.

A única diferença entre os algoritmos MGetW (Algoritmo 18) e GETW (Algoritmo 15) é a Linha 5. O Algoritmo 18 chama o método GetClosestP nessa linha. O algoritmo GetClosestP encontra a probabilidade de custo-limite imediatamente predecessora que já foi calculada (i.e., $\left.p_{D D}^{*}\left(\cdot, \theta_{x}\right)^{\prime}\right)$ tal que $\theta_{x} \leq \theta-c$, que é necessária quando $\theta-c=\theta_{y} \notin \Theta_{r}$.

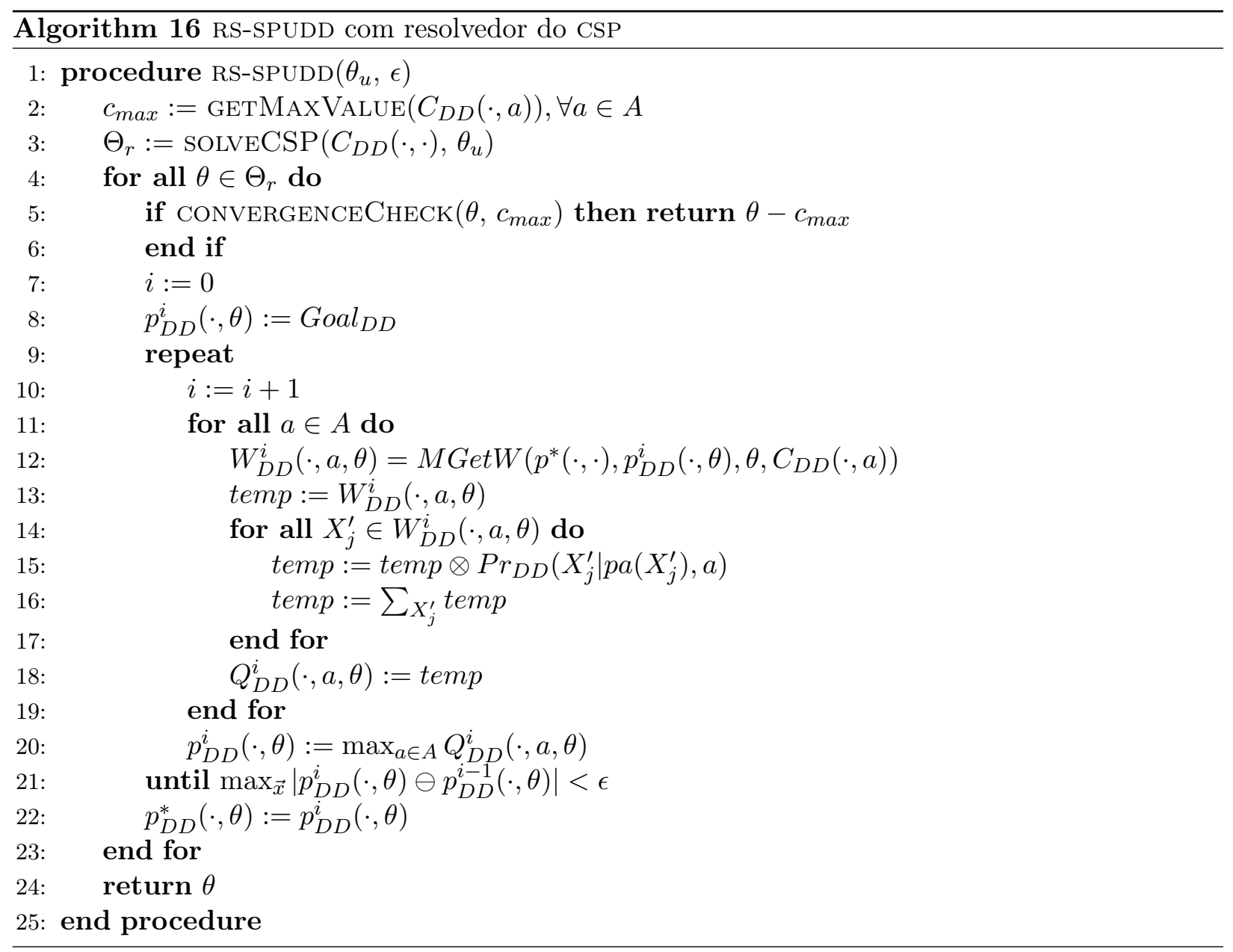

Adicionalmente, note que o algoritmo RS-SPUDD pode ser otimizado em domínios que não possuem nenhum custo imediato igual a zero para qualquer estado não meta. Nessa situação, é possível remover o terceiro caso na Equação 8.4 e então o cálculo de $p_{D D}^{i+1}$ seria independente de $p_{D D}^{i}$. Como consequência não é necessário realizar várias iterações até convergência (Linhas 9-21 no Algoritmo 16), portanto nesse caso uma iteração é suficiente. 

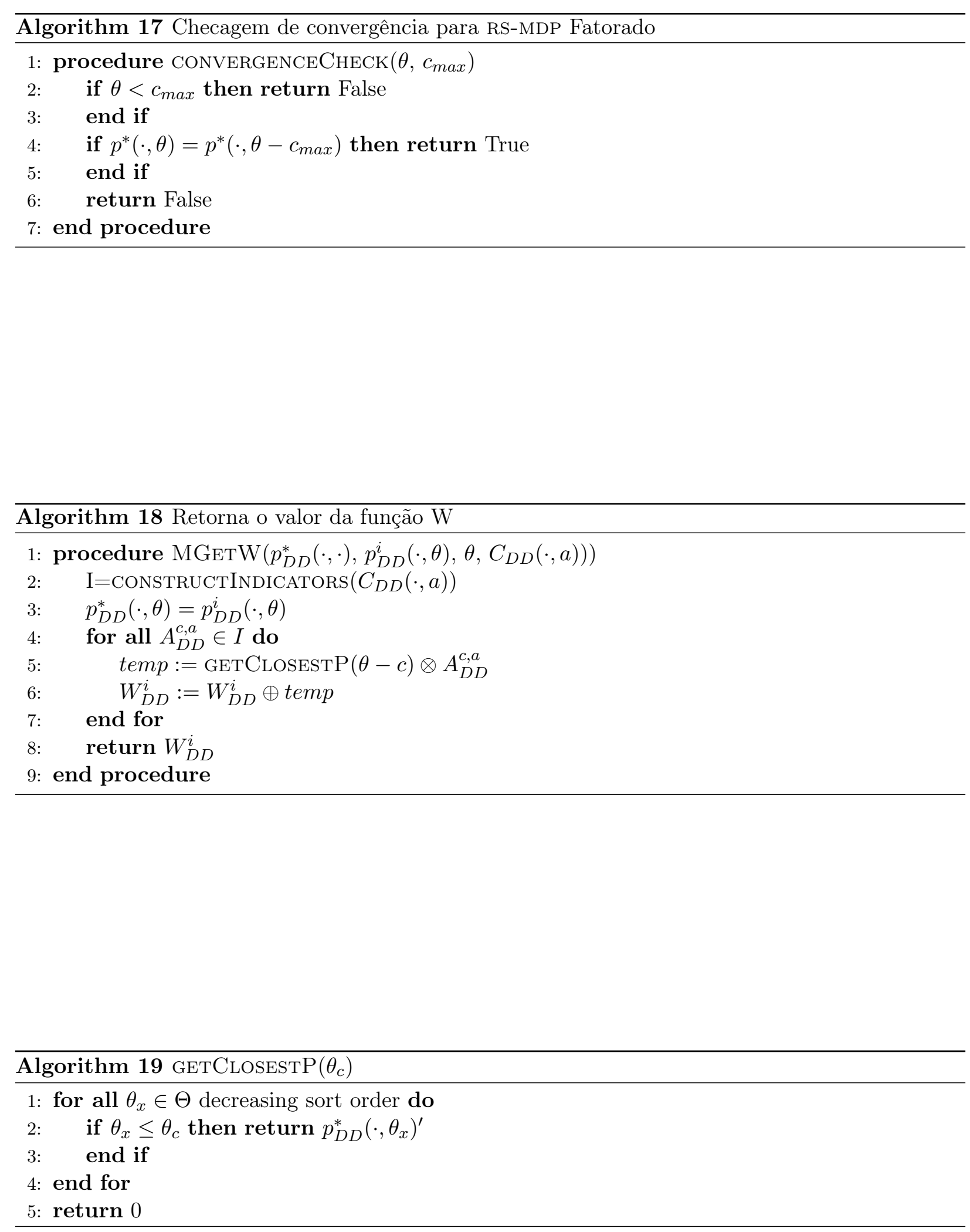


\section{Capítulo 9}

\section{Processos de Decisão Markovianos Híbridos Sensíveis ao Risco}

Neste capítulo são introduzidos os Processos de Decisão Markovianos Híbridos Sensíveis ao Risco, que são MDPs Sensíveis ao Risco que incluem variáveis contínuas e discretas, além do orçamento dado pelo usuário. Além disso, é mostrado que é possível resolver esse tipo de problemas com algoritmos existentes na literatura fazendo algumas mudanças na modelagem.

\subsection{Definição Formal}

Definição 19. Um hmDP Sensível ao Risco pode ser definido pela tupla $<S, G, A, C, P_{r}, \theta_{u}>$ tal que:

- $S$ é o conjunto de estados $S=\vec{X} \times \vec{Y}$. O vetor $\vec{X}$ é um vetor de variáveis de estado booleanas $\left(X_{1}, \ldots, X_{n}\right)$ de comprimento $n$, uma atribuição de $\vec{X}$ é um vetor $\vec{x}=\left(x_{1}, \ldots, x_{n}\right)$ em que $x_{i} \in\{0,1\}$ é o valor da variável $X_{i}$. O vetor $\vec{Y}$ é um vetor de variáveis de estado contínuas $\left(Y_{1}, \ldots, Y_{m}\right)$ de comprimento $m$, uma atribuição de $\vec{Y}$ é um vetor $\vec{y}=\left(y_{1}, \ldots, y_{m}\right)$ em que $y_{j} \in\left[L_{j}, U_{j}\right], L_{j} \leq U_{j} \in \mathbb{R}$. Portanto, um estado $s$ é uma atribuição das variáveis $s=(\vec{x}, \vec{y})=$ $\left(x_{1}, \ldots, x_{n}, y_{1}, \ldots, y_{m}\right) \in \vec{X} \times \vec{Y}$;

- $G$ é um conjunto finito de estados meta absorventes, $G \subset S$;

- $A$ é um conjunto finito de ações;

- $C(\vec{x}, \vec{y}, a)$ é uma função de custo arbitrária (que pode ser um valor real, ou uma função linear por partes ou polinomial por partes).

- $P_{r}$ é uma função de transição conforme definido para HMDPs;

- $\theta_{u}$ é o orçamento limite dado pelo usuário.

A função de otimilidade para HMDPs Sensíveis ao Risco é definida como:

$$
P^{*}(\vec{x}, \vec{y}, \theta)=\max _{a \in A} \sum_{\vec{x}^{\prime} \in X} \int_{\vec{y}^{\prime} \in \vec{Y}} \begin{cases}0 & \text { se } C(\vec{x}, \vec{y}, a)>\theta, \\ 1 & \text { se } C(\vec{x}, \vec{y}, a) \leq \theta \text { e }(\vec{x}, \vec{y}) \in G, \\ P_{r}\left(\vec{x}^{\prime}, \vec{y}^{\prime} \mid \vec{x}, \vec{y}, a\right) P^{*}\left(\vec{x}^{\prime}, \vec{y}^{\prime}, \theta-C(\vec{x}, \vec{y}, a)\right) & \text { se } C(\vec{x}, \vec{y}, a) \leq \theta \text { e }(\vec{x}, \vec{y}) \notin G .\end{cases}
$$

\subsection{Resolução de HMDP Sensíveis ao Risco utilizando HMDPs}

É possível resolver um HMDP Sensível ao Risco utilizando HMDPs $\left.\left(<S, A, P_{r}, R\right\rangle\right)$ como definido na Seção 6.1 com um conjunto de estados meta $G$ do seguinte modo: 
- O conjunto de estados $S$ é estendido com a variável de orçamento restante $\Gamma$, i.e. $S=\vec{X} \times \vec{Y}_{r}$, em que o vetor $\vec{Y}_{r}=\left(Y_{1}, \ldots, Y_{m}, \Gamma\right)$ é o vetor de variáveis de estado contínuas $Y$ estendido com a variável $\Gamma$. Uma atribuição de $\vec{Y}_{r}$ é um vetor $\vec{y}_{r}=\vec{y} \cup \theta=\left(y_{1}, \ldots, y_{m}, \theta\right)$ em que $y_{j} \in\left[L_{j}, U_{j}\right], L_{j} \leq U_{j} \in \mathbb{R}$ e $\theta \in\left[0, \theta_{u}\right]$. Portanto, um estado $s$ é uma atribuição das variáveis $s=\left(\vec{x}, \vec{y}_{r}\right)=\left(x_{1}, \ldots, x_{n}, y_{1}, \ldots, y_{m}, \theta\right) \in \vec{X} \times \vec{Y}_{r}$

- O conjunto de estados meta $G$ é estendido com a variável de orçamento restante, i.e. $G \subset$ $\vec{X} \times \vec{Y}_{r}$

- A função de transição $P_{r}$ é estendida com a inclusão da distribuição $P_{r}\left(\theta^{\prime} \mid \vec{x}, \vec{y}_{r}, a\right)=\delta\left(\theta^{\prime}-\right.$ $\left.g\left(\vec{x}, \vec{y}_{r}, a\right)\right)$, em que $g$ é definida em termos da função de custo $C$ :

$$
g\left(\vec{x}, \vec{y}_{r}, a\right)= \begin{cases}\left(\vec{x}, \vec{y}_{r}\right) \notin G \wedge \theta \geq C\left(\vec{x}, \vec{y}_{r}, a\right): & \theta-C\left(\vec{x}, \vec{y}_{r}, a\right) \\ \left(\vec{x}, \vec{y}_{r}\right) \notin G \wedge \theta<C\left(\vec{x}, \vec{y}_{r}, a\right): & -\infty \\ \left(\vec{x}, \vec{y}_{r}\right) \in G: & \theta\end{cases}
$$

A Equação 9.2 define os orçamentos futuros $\theta^{\prime}$ em função do orçamento corrente $\theta$ e da função de custo. No primeiro caso, o estado corrente não é um estado meta e a ação $a$ é aplicável dado o orçamento corrente $\theta$ (i.e. $\theta \geq C\left(\vec{x}, \vec{y}_{r}, a\right)$ ), portanto o orçamento futuro é dado por $\theta^{\prime}=\theta-C\left(\vec{x}, \vec{y}_{r}, a\right)$. No segundo caso, o estado corrente não meta não possuí orçamento suficiente para a aplicar a ação $a$ (i.e. $\theta<C\left(\vec{x}, \vec{y}_{r}, a\right)$ ), portanto transitamos para um estado inválido (representado por $-\infty$ ). No terceiro caso, o estado corrente é um estado meta (absorvente), logo o orçamento futuro se mantém inalterado (i.e. $\theta^{\prime}=\theta$ );

- A função de recompensa é definida como $R\left(\vec{x}, \vec{y}_{r}\right)=0, \forall\left(\vec{x}, \vec{y}_{r}\right) \in S$, pois o objetivo é maximizar probabilidades.

É possível resolver a Equação 9.1 com a seguinte Equação de Otimalidade de Bellman para HMDPs com as mudanças descritas:

$$
f^{*}\left(\vec{x}, \vec{y}_{r}\right)=\max _{a}\left[R\left(\vec{x}, \vec{y}_{r}, a\right)+\sum_{\vec{x}^{\prime} \in \vec{X}} \int_{\vec{y}_{r}^{\prime} \in \vec{Y}}\left\{\begin{array}{ll}
1 & \text { se }\left(\vec{x}, \vec{y}_{r}\right) \in G \\
f^{*}\left(\vec{x}^{\prime}, \vec{y}_{r}^{\prime}\right) * P_{r}\left(\vec{x}^{\prime}, \vec{y}_{r}^{\prime} \mid \vec{x}, \vec{y}_{r}, a\right) d \vec{y}_{r}^{\prime} & \text { caso contrário }
\end{array}\right]\right.
$$

É possível verificar que essa equação mantém os três casos da Equação 9.1:

1. Estados com orçamentos insuficiente têm valor ótimo igual a zero. Isso se mantêm verdadeiro, pois como definido anteriormente, estados com valor $\theta^{\prime}<0$ transitam para um estado inválido (representado por $-\infty$ ), portanto na equação temos apenas a recompensa, que por definição é 0 .

2. Estados meta possuem valores ótimos igual a um o que é definido pelo primeiro caso da Equação 9.3.

3. Estados não-meta com orçamento suficiente possuem valores ótimos definidos pelo produto entre a transição e valores ótimos de seus estados sucessores com desconto no orçamento disponível dado pela função de custo. Note que, a função de recompensa tem valor zero. Portanto, para estados não-meta, a equação 9.3 é simplificada para:

$$
\max _{a}\left[\sum_{\vec{x}^{\prime} \in \vec{X}} \int_{\vec{y}_{r}^{\prime} \in \vec{Y}} f^{*}\left(\vec{x}^{\prime}, \vec{y}_{r}^{\prime}\right) * P_{r}\left(\vec{x}^{\prime}, \vec{y}_{r}^{\prime} \mid \vec{x}, \vec{y}_{r}, a\right) d \vec{y}_{r}^{\prime}\right]
$$

ou seja, tais estados são definidos pelo mesmo produto necessário para propagar os valores ótimos dos estados sucessores. Além disso, a definição de transição dada para $\theta^{\prime}$ nesses estados mantêm o mesmo desconto necessário (i.e. $\theta^{\prime}=\theta-C\left(\vec{x}, \vec{y}_{r}, a\right)$ como definido no primeiro caso da Equação 9.2). 
A abordagem apresentada aqui permite duas importantes generalizações para resolver problemas com sensibilidade ao risco mais gerais: (i) estados são representados por variáveis booleanas e contínuas; e (ii) a função de custo permite definições mais complexas (linear e polinomial) diferente das abordagens anteriores que são restritas a funções que definem valores reais para cada par estado e ação.

Assim, é possível utilizar o algoritmo SDP apresentado na Seção ?? para resolver o HMDP Sensível ao Risco inicializando a função $f^{0}$ com 1 para os estados metas. 


\section{Capítulo 10}

\section{Resultados}

Os experimentos foram separados em duas seções. Na primeira, o tempo de convergência e o consumo de memória dos algoritmos RS-SPUDD, TVI-DP e ITVI-DP são avaliados em dois diferentes domínios do IPPC 2011: domínio do Robô Navegador e domínio SysAdmin.

Na segunda parte, o tempo de convergência do algoritmo para RS-MDP híbrido é avaliado em dois domínios: domínio do Robô Navegador e domínio Mars Rover. O consumo de tempo do algoritmo para RS-MDP híbrido é comparado com os demais algoritmos avaliados na primeira seção no domínio do Robô Navegador, que não tem variáveis contínuas. Uma vez que não existe um outro algoritmo para resolver RS-MDP híbrido, apenas o algoritmo proposto é avaliado no segundo domínio que contém variáveis contínuas.

Nos experimentos foi usada uma máquina virtual com 4 processadores de $3.5 \mathrm{GHz}$ e $8 \mathrm{~GB}$ de memória. $\mathrm{O}$ erro de convergência foi definido como $\epsilon=0.01 \mathrm{em}$ todos as instâncias de teste e o orçamento foi definido como o maior valor para cada teste em que pelo menos um dos algoritmos foi capaz de resolver, dado os limites de memória impostos nos testes realizados (máximo de 256 $\mathrm{MB})$.

\subsection{Avaliação dos Algoritmos para RS-MDPs}

Nessa seção, os domínios utilizados na avaliação dos algoritmos RS-SPUDD, TVI-DP e ITVI-DP são descritos em detalhes. Posteriormente, os resultados de tempo de convergência e consumo de memória de cada algoritmo nesses domínios são apresentados e discutidos.

\subsubsection{Domínio do Robô Navegador}

Robô Navegador é um domínio de planejamento probabilístico bem conhecido que envolve um robô móvel que deve navegar de uma posição inicial até uma posição meta em uma grade de tamanho $n \times m$. O robô pode se mover para qualquer posição adjacente da sua posição atual, porém qualquer movimento realizado, exceto na primeira e última linhas, têm uma probabilidade de fazer o robô desaparecer (ou quebrar). Essa probabilidade aumenta conforme o robô se aproxima da coluna em que está a meta. Além disso, a cada movimento é atribuído um valor de custo que depende da posição atual do robô e da direção do movimento.

O domínio do robô navegador pode ser especificado usando codificação binária das variáveis de estado. O espaço de estados é definido por $S=\{\vec{x}, \vec{y}, d\}$ tal que $x_{i}, y_{i}, d \in\{0,1\}$, e $\vec{x}=(n)_{2}, \vec{y}=$ $(m)_{2}$ e $d$ são respectivamente a linha corrente, coluna corrente e o valor da variável que representa a existência do robô ${ }^{1}$. O conjunto de ações $A=(i, j)$ em que $i, j \in\{-1,0,1\}, i=0 \Leftrightarrow j \neq 0$ e $i \neq 0 \Leftrightarrow j=0$ define quatro ações, em que cada um move o robô para uma célula adjacente

\footnotetext{
${ }^{1}(z)_{2}$ representa o número inteiro em sistema binário e $(\vec{w})_{10}$ representa o vetor em sistema decimal.
} 
diferente. A ação de andar em qualquer direção é determinística e segue a seguinte equação:

$$
\begin{gathered}
\vec{x}^{\prime}=\left((\vec{x})_{10}+i\right)_{2} *(1-d) \\
\vec{y}^{\prime}=\left((\vec{y})_{10}+j\right)_{2} *(1-d),
\end{gathered}
$$

porém existe a probabilidade do robô desaparecer em algumas células. Essa probabilidade é definida pela seguinte equação:

$$
\begin{array}{r}
T\left(d^{\prime} \mid \vec{x}, \vec{y}, d, a\right)=(1-d) *\left((\vec{x})_{10} \neq 0\right) *\left((\vec{x})_{10} \neq n\right)\left(1-\left(\max P r o b+(\vec{y})_{10}\right.\right. \\
*((\operatorname{minProb}-\max P r o b) /(m-1)))),
\end{array}
$$

em que minProb e maxProb são respectivamente a probabilidade mínima e máxima do robô desaparecer. Finalmente, a função de custo é definida como:

$$
C(\vec{x}, \vec{y}, d, a)= \begin{cases}0 & \text { se }\left((\vec{x})_{10}=n \wedge(\vec{y})_{10}=m\right) \vee d=1 \\ \alpha & \text { se } i=1 \\ 2.0 * \alpha & \text { se } i=-1 \\ \lfloor 1.5 * \alpha\rfloor & \text { Caso contrário }\end{cases}
$$

em que $\alpha$ é um custo fixo associado a ação de rotacionar o robô em direção a sala desejada assumindo que a frente do robô sempre está direcionada para cima. O número de estados aumentados de um dada instância está em função do tamanho da grade e $\theta_{u}$ (i.e., $\left.N=\theta_{u} *(\vec{x})_{10} *(\vec{y})_{10}+1\right)$. Nos experimentos é usado $\alpha=10$.

Vale a pena ressaltar que essa definição do domínio é compacta no número de variáveis necessárias, sendo que apenas $\log _{2}(n)+\log _{2}(m)+1$ variáveis são utilizadas. Tais variáveis são dividas em: (i) $\log _{2}(n)$ variáveis $X_{1}, X_{2}, \ldots, X_{\log _{2} n}$ que representam as linhas da grade que são $n$ no total; (ii) $\log _{2} m$ variáveis $Y_{1}, Y_{2}, \ldots, Y_{\log _{2} m}$ que representam as colunas da grade que são $m$ no total; e (iii) 1 variável de controle de existência do robô $D$.

Na Figura 10.1 é apresentado um exemplo simples do domínio do robô navegador com uma grade $4 \times 4$ e coordenada meta $(3,3)$. A Figura 10.1 (a) ilustra essa grade com as coordenadas. É importante ressaltar que na modelagem proposta para esse domínio, as coordenadas da grade são representadas por valores binários. Por exemplo, a coordenada $(2,1)$ é representada por $\left(x_{2}=\right.$ $\left.1, x_{1}=0 ; y_{2}=0, y_{1}=1\right)$.

A Figura 10.1(b) ilustra um exemplo de DBN para a ação ir-para-cima. Note que DBNs mapeiam dependências causais entre variáveis como podemos ver na Figura 10.1(b) em que a probabilidade do robô desaparecer no próximo estado $\left(D^{\prime}\right)$ depende de todos os demais fatores, ou seja, depende da existência e da posição corrente do robô. Em contraste, a linha em que o robô se encontrará no próximo estado $\left(Y_{i}^{\prime}\right)$ depende somente da existência e da linha corrente do robô. Essas particularidades são capturadas pela representação em ADDs, como mostrada no ADD da função de transição da variável $X_{1}^{\prime}$ com a ação ir-para-cima (Figura 10.1(c)).

A função de custo que depende de todas as variáveis é ilustrada na Figura 10.1(d) da esquerda e sua respectiva representação em ADD na Figura 10.1(d) da direita. Na função de custo apresentada, o robô é penalizado em 1 por cada transição realizada e não é penalizado quando está na posição objetivo.

\subsubsection{Domínio SysAdmin}

No domínio SysAdmin, $n$ computadores são conectados em uma rede por diferentes topologias e cada computador pode estar ou não funcionando em cada espaço de tempo. O administrador da rede tem como objetivo manter todos os computadores funcionando ao mesmo tempo e pode reiniciar um computador por vez a cada espaço de tempo.

O conjunto de estados é definido por $S=\{\vec{c}\}$, em que $c_{i} \in\{0,1\}$ representa se o $i$-ésimo 


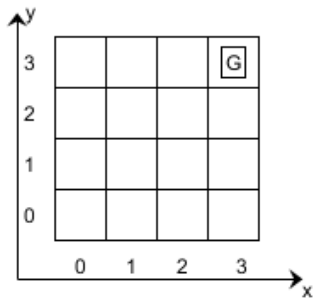

Grade

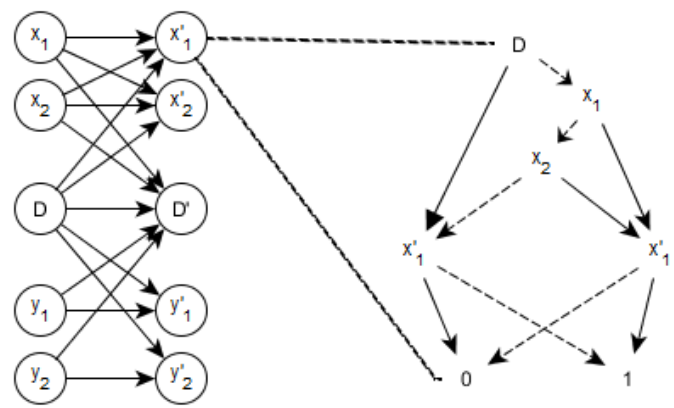

DBN
Representaçäo em ADD

(c)

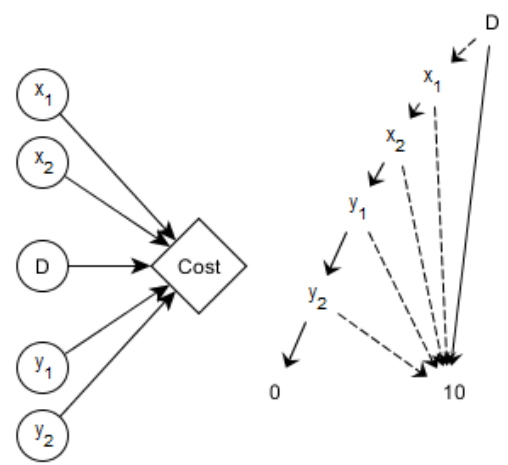

Rede de custo

(d)

Figura 10.1: Exemplo simples do robô navegador: (a) grade com coordenadas $X$ e $Y$; (b) DBN para a ação ir-para-cima em que as coordenadas são expressas em binário; (c) Representação em ADD da CPT para a variável $X_{1}^{\prime}$; (d) Representação em ADD do custo imediato com $\alpha=10$.

computador está funcionando (1) ou não (0). O conjunto de ações $A$ é composto por $n$ ações: $\operatorname{reboot}\left(c_{1}\right), \ldots, \operatorname{reboot}\left(c_{n}\right)$ e notreboot(), que significa respectivamente reiniciar a maquina $c_{1}, \ldots, c_{n}$ e não reiniciar nenhuma máquina. A função de transição é definida por:

$$
T\left(c_{i}^{\prime} \mid \vec{c}, a\right)= \begin{cases}1 & \text { se } a=\operatorname{reboot}\left(c_{i}\right) \\ 0.95 \cdot \frac{\left|\left\{c_{j} \mid j \neq i \wedge c_{j}=1 \wedge \operatorname{Conm}\left(c_{i}, c_{j}\right)\right\}\right|+1}{\left|\left\{c_{j} \mid j \neq i \wedge \operatorname{Conm}\left(c_{i}, c_{j}\right)\right\}\right|+1} & \text { se } a \neq \operatorname{reboot}\left(c_{i}\right) \wedge c_{i}=1 \\ 0.05 \cdot \frac{\left|\left\{c_{j} \mid j \neq i \wedge c_{j}=1 \wedge \operatorname{Conm}\left(c_{i}, c_{j}\right)\right\}\right|+1}{\left|\left\{c_{j} \mid j \neq i \wedge \operatorname{Conm}\left(c_{i}, c_{j}\right)\right\}\right|+1} & \text { se } a \neq \operatorname{reboot}\left(c_{i}\right) \wedge c_{i}=0,\end{cases}
$$

em que $\operatorname{Conm}\left(c_{i}, c_{j}\right)$ é verdade (1) quando os computadores $c_{i}$ e $c_{j}$ estão diretamente conectados e falso (0), caso contrário. Observe que esse domínio é extremamente denso, já que a maioria dos estados é alcançável em um único passo.

A função de custo é definida como:

$$
C(\vec{c}, a)=\text { cost }_{\text {reboot }}+\sum_{c_{j} \in \vec{c}}\left(1-c_{j}\right) * \operatorname{level}\left(c_{j}\right) * \text { cost }_{l},
$$

em que cost $_{\text {reboot }}$ é o custo de reiniciar uma máquina, level $\left(c_{j}\right): c_{j} \rightarrow\{1,2,3\}$ é uma função (chamada de nível crítico) que associa um número (entre 1 e 3) para cada computador dado sua respectiva importância para a rede (e.g. computadores com informações cruciais possuem maior valor) e cost $_{l}$ é um custo associado ao manter um computador desligado de nível crítico $l$.

Na Figura 10.2 é mostrado um exemplo de DBN para a ação de reiniciar o computador 1 (Figura 
10.2 (b)) e a representação em ADD da CPT para as variáveis $C_{1}$ e $C_{2}$ (Figura 10.2 (c)) e da função de custo imediato (Figura $10.2(\mathrm{~d})$ ).

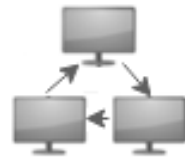

Rede de Computadores

(a)

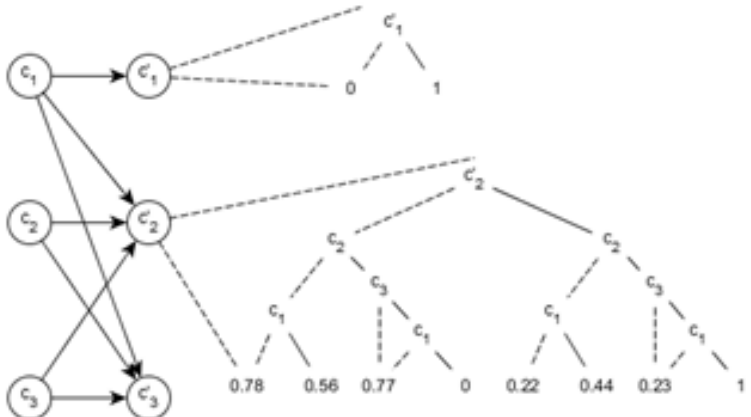

DBN

(b)

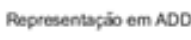

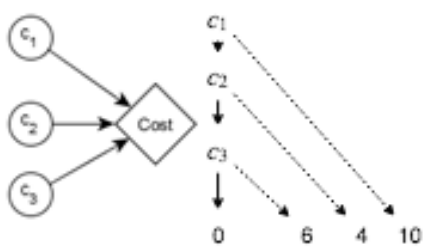

Funclio de custo

Figura 10.2: Exemplo simples do SysAdmin: (a) Rede de computadores interconectados; (b) DBN para a ação de reiniciar computador 1; (c) Representação em ADD da CPT para as variáveis $C_{1}$ e $C_{2}$; e (d) Representação em ADD do custo imediato em que level $\left(c_{j}\right)=j$, cost $t_{\text {reboot }}=9$ e cost $l_{l}=2$.

O número de estados aumentados de uma dada instância está em função da quantidade de computadores na rede e $\theta_{u}$ (i.e. $N=\theta_{u} *|\vec{c}|^{2}$ ). Nos experimentos a topologia da rede é definida como um anel (Figura 10.2 (a)), o custo de reiniciar é dado por cost $_{\text {reboot }}=9$, o custo por nível crítico é definido como cost $_{l}=2$ e o nível crítico de cada computador é definido aleatoriamente.

\subsubsection{Análise do Tempo de Convergência}

As Figuras 10.3 e 10.4 ilustram o tempo de convergência global dos algoritmos TVI-DP, ITVI-DP e RS-SPUDD para diferentes instâncias dos domínios do Robô Navegador e SysAdmin, respectivamente. O orçamento usado foi $\theta_{u}=500$.

As Figuras 10.3 e 10.4 mostram que o algoritmo TVI-DP não conseguiu resolver instâncias maiores ou iguais que $32 \times 32$ (com 512001 estados aumentados) e 8 computadores (com 128000 estados aumentados) para o domínio do Robô Navegador e SysAdmin, respectivamente. Em compensação, o algoritmo ITVI-DP foi capaz de resolver mais instâncias que o algoritmo TVI-DP, porém o algoritmo ITVI-DP não foi capaz de resolver todos os problemas que o algoritmo RS-SPUDD conseguiu.

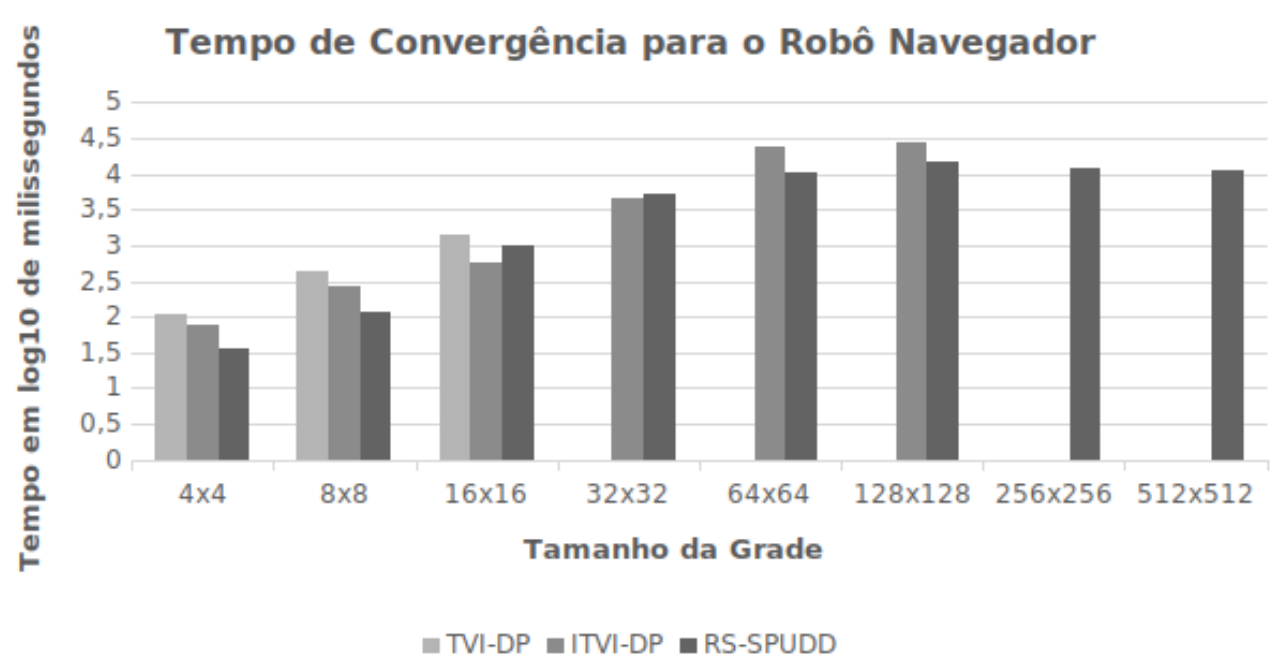

Figura 10.3: Tempo de convergência em $\log _{10}$ de milissegundos para o domínio do Robô Navegador. 


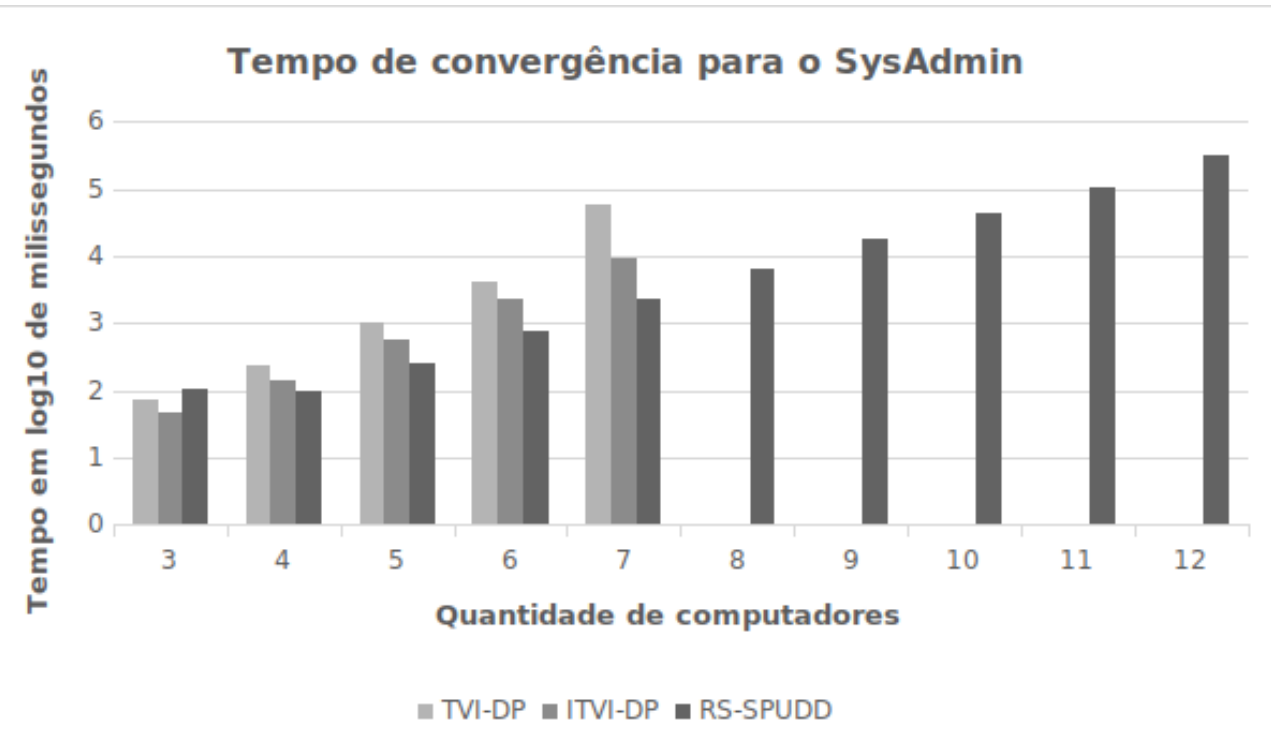

Figura 10.4: Tempo de convergência em $\log _{10}$ de milissegundos para o domínio SysAdmin.

Além disso, em quase todas as instâncias de ambos os domínios, o algoritmo proposto RSSPUDD foi mais rápido que TVI-DP and ITVI-DP. Apenas em três instâncias (que são pequenas) o algoritmo proposto não foi mais rápido. Isso pode ter sido causado por uma sobrecarga para calcular os orçamentos válidos e criar os ADDs. Porém, em geral, RS-SPUDD é claramente melhor para instâncias maiores.

Para as primeiras três instâncias do Robô Navegador, os algoritmos ITVI-DP e RS-SPUDD detectaram o valor de $\theta_{\text {min }}$. Os valores de $\theta_{\min }$ encontrados são 66, 151 e 330, respectivamente. Para as demais instâncias, $\theta_{u}=500$ não foi grande o suficiente para as probabilidades de custo-limite convergirem, e portanto, os algoritmos ITVI-DP e RS-SPUDD não retornaram $\theta_{\min }$.

O domínio SysAdmin tende a probabilidade máxima de 1 quando o orçamento tende a infinito, logo dado um orçamento suficientemente grande $\theta_{u}$, não há $\theta_{\min }$ porque o agente sempre tenta consumir o máximo de orçamento possível.

As Figuras 10.5 e 10.6 ilustram o tempo de convergência para instâncias do Robô Navegador com tamanho de grade de 256x256 e instâncias do SystAdmin com 9 computadores em rede, respectivamente. Tais tamanhos de grade foram selecionados para garantir que os algoritmos TVI-DP ou ITVI-DP fossem capazes de resolver algumas das instâncias. O valor de $\theta_{u}$ varia de 50 até $500 \mathrm{com}$ incrementos de 50. Para as instâncias do Robô Navegador, os resultados mostram que o algoritmo TVI-DP não foi capaz de resolver nenhuma instância que o algoritmo RS-SPUDD resolveu, enquanto que o algoritmo ITVI-DP não foi capaz de resolver 2 instâncias. Além disso, para o dóminio SysAdmin o algoritmo TVI-DP foi capaz de resolver somente as primeiras três instâncias enquanto que o algoritmo ITVI-DP resolveu somente as quatro primeiras.

Em domínios para o qual o conjunto de orçamentos válidos é igual ao conjunto considerado pelo TVI-DP, o único overhead é resolver a Equação 7.2. Para domínios com alta dependência entre variáveis de estado é esperado que o algoritmo RS-SPUDD tenha o mesmo comportamento que o algoritmo SPUDD, quando comparado com o algoritmo iteração de valor simples: $o$ overhead envolvido em criar $\mathrm{ADD} s$ não é muito severo, e tende a diminuir à media que o tamanho dos problemas aumenta [HSAHB99].

\subsubsection{Análise do Consumo de Memória}

As Figuras 10.7 e 10.8 ilustram o consumo de memória em Kbytes dos algoritmos TVI-DP, ITVI-DP e RS-SPUDD para instâncias diferentes para os domínios do Robô Navegador e SysAdmin, respectivamente. O orçamento usado foi $\theta_{u}=500$.

O algoritmo RS-SPUDD foi o único algoritmo capaz de resolver as maiores instâncias de ambos 


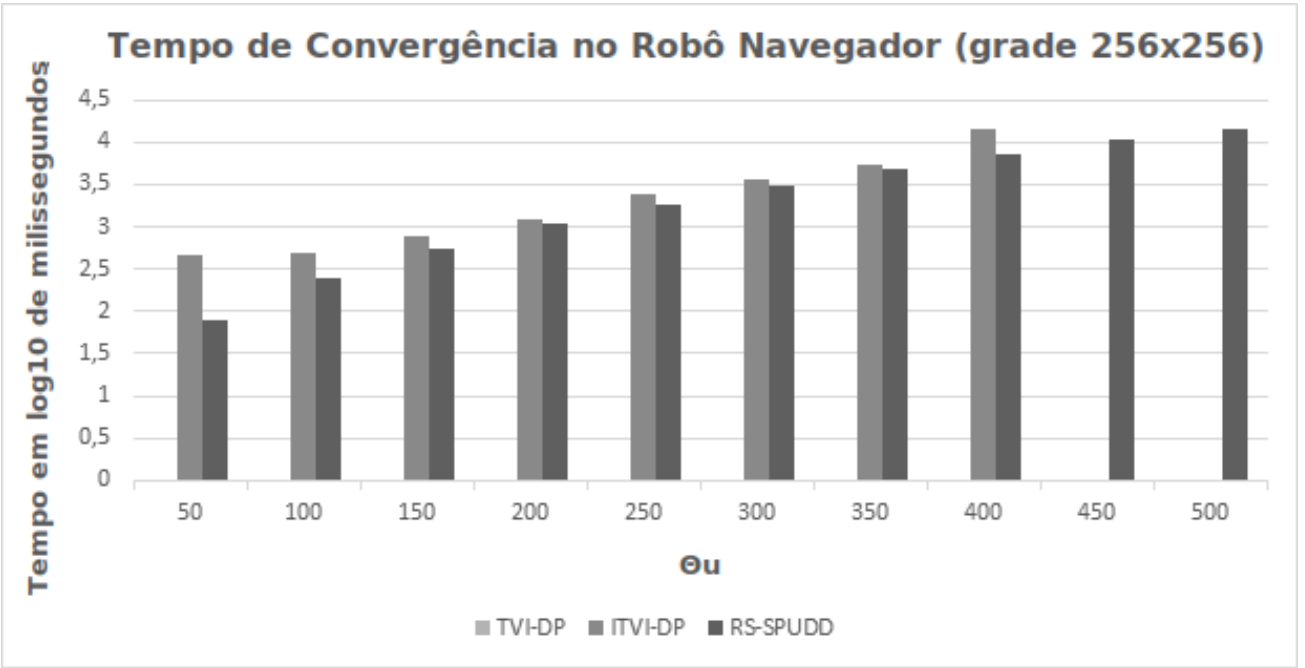

Figura 10.5: Tempo de convergência para o domínio do Robô Navegador com $\theta_{u}$ variando entre 50 e 500.

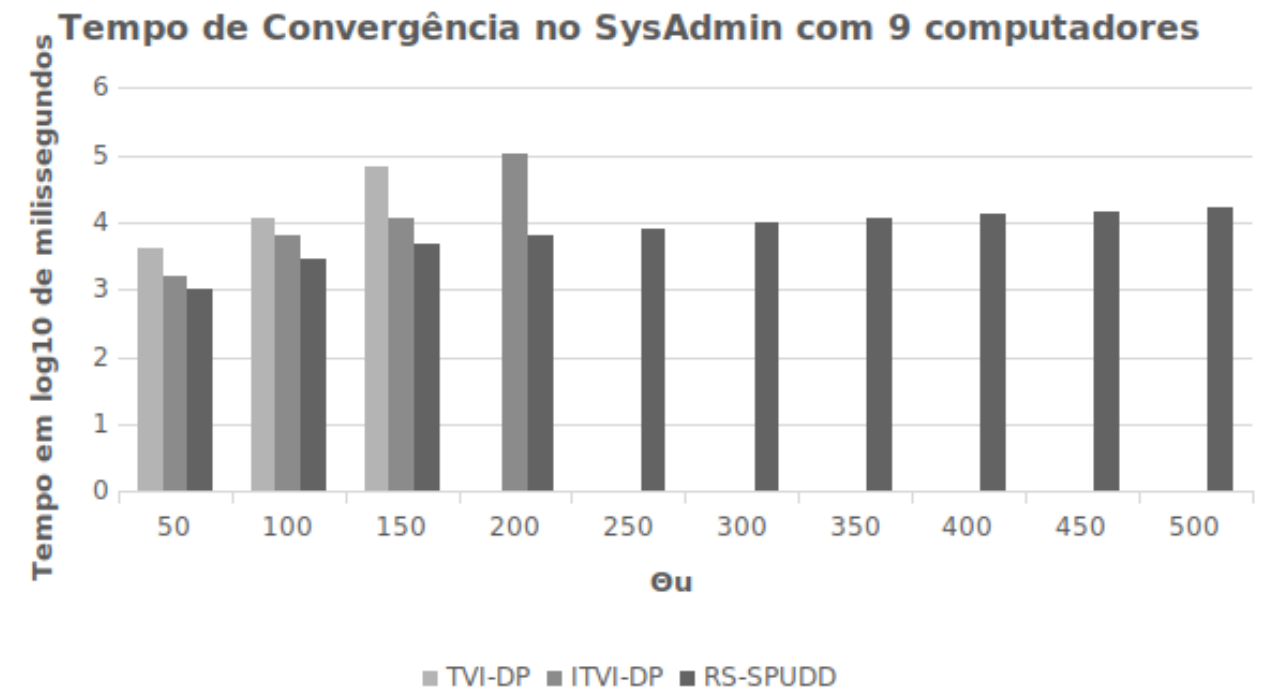

Figura 10.6: Tempo de convergência para o domínio do SysAdmin com $\theta_{u}$ variando entre 50 e 500.

os domínios testados sem exceder o limite máximo de memória atribuído a eles.

A Figura 10.7 revela que para todas as instâncias, o algoritmo proposto (RS-SPUDD) demanda muito menos memória que o algoritmo ITVI-DP (o segundo melhor algoritmo testado), as únicas exceções foram na segunda instância do domínio do Robô Navegador (grade 8x8) em que ambos os algoritmos RS-SPUDD e ITVI-DP empataram no consumo de memória.

A Figura 10.8 mostra que para todas as instâncias menores, o algoritmo RS-SPUDD teve o mesmo consumo de memória que o algoritmo ITVI-DP enquanto que para todas as instâncias com 6 ou mais computadores, o RS-SPUDD demanda muito menos memória.

\subsection{Avaliação do Algoritmo para RS-MDPs Híbridos}

Nesta seção, os domínios utilizados na avaliação do algoritmo para RS-MDPs híbridos são descritos em detalhes. Posteriormente, os resultados de tempo de convergência para esse algoritmo são apresentados e discutidos. 


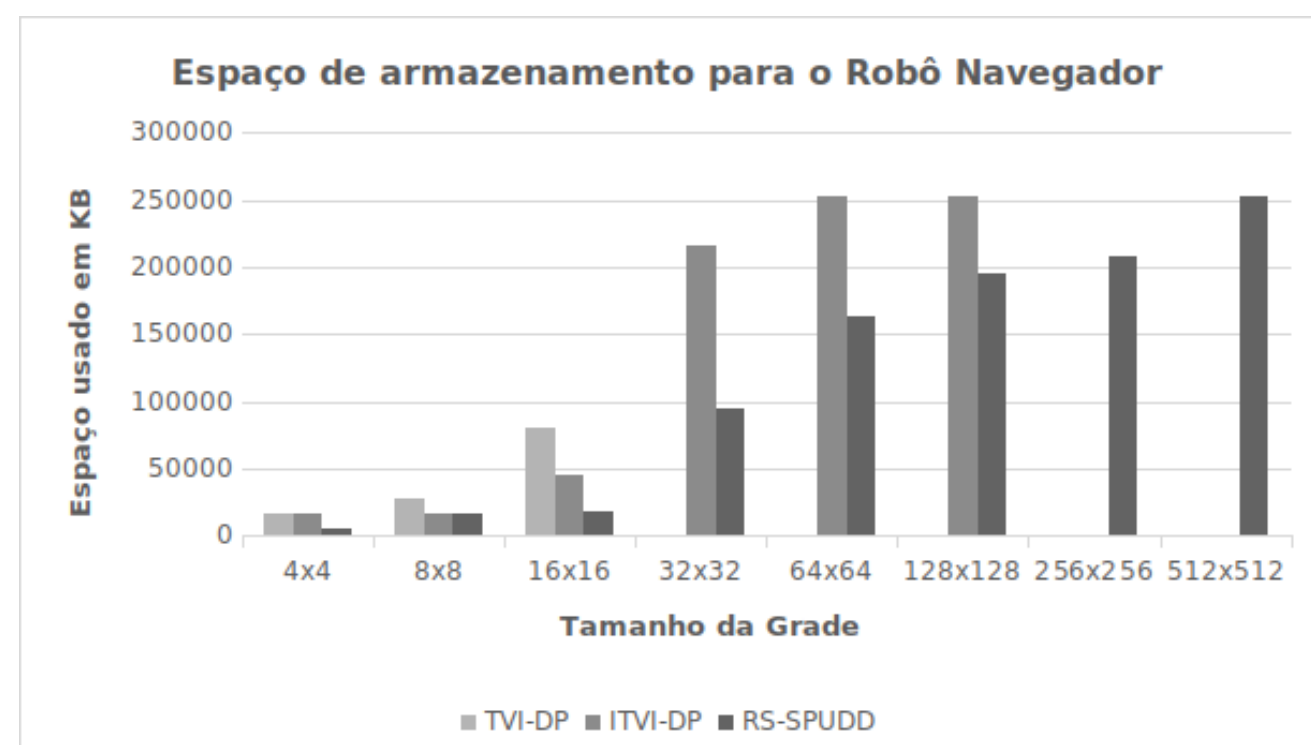

Figura 10.7: Consumo de memória em Kbytes para o dominio do Robô Navegador.

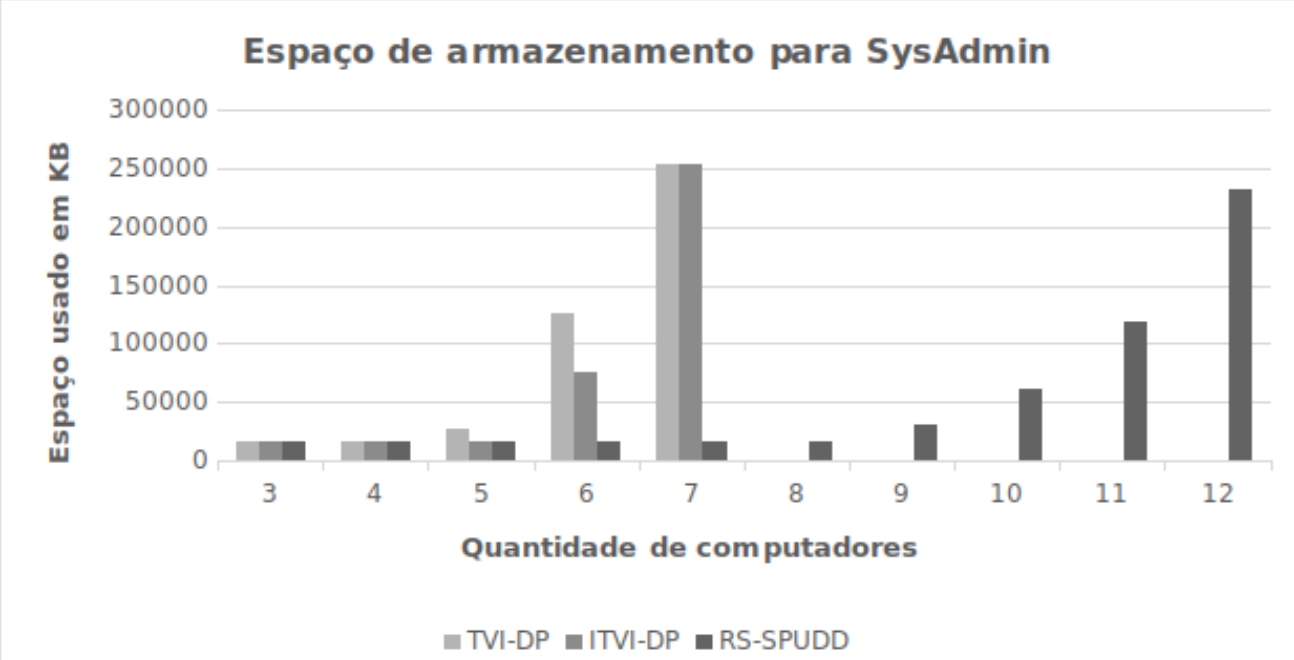

Figura 10.8: Consumo de memória em Kbytes para o dominio SysAdmin.

\subsubsection{Domínio do Robô Navegador}

Domínios modelados como RS-MDP fatorados podem ser modelados como HMDP Sensível ao Risco. A seguir, são discutidas duas formas de representar o domínio do Robô Navegador como um HMDP Sensível ao Risco.

Considere que, para esse domínio o orçamento definido pelo usuário está diretamente relacionado com a quantidade de bateria do robô. Duas opções possíveis para representar esse problema como um HMDP Sensível ao Risco são:

1. Representar cada posição da grade por um conjunto de variáveis booleanas de tamanho $\log _{2}(n *$ $m$ ) e a variável contínua $\Gamma=E$ que mantém a quantidade de bateria restante do robô. Essa representação é semelhante a representação descrita anteriormente desse domínio com RS-MDP fatorado.

2. Representar as posições da grade com duas variáveis contínuas $Y_{\text {linha }}$ e $Y_{\text {coluna }}$, além da variável contínua $\Gamma=E$ para a quantidade de bateria restante.

Os experimentos realizados nesta dissertação e apresentados a seguir utilizam a primeira representação que segue a mesma definição para esse domínio à apresentada para RS-MDP fatorado 
com a adição da variável contínua $\Gamma$ (i.e. o espaço de estados é definido por $S=X \times Y=$ $\left.\left\{X_{1}, \ldots, X_{n}, Y_{1}, \ldots, Y_{m}, D\right\} \times\{\Gamma\}\right)$. A transição para $e^{\prime}$ é dada por $P_{r}\left(e^{\prime} \mid \vec{x}, \vec{y}, e, a\right)=\delta\left(e^{\prime}-g(\vec{x}, \vec{y}, e, a)\right)$, em que $g$ é definida por:

$$
g(\vec{x}, \vec{y}, e, a)= \begin{cases}\left((\vec{x})_{10} \neq n \vee(\vec{y})_{10} \neq m\right) \wedge d=0 \wedge e \geq C(\vec{x}, \vec{y}, a): & e-C(\vec{x}, \vec{y}, a) \\ \left(\left((\vec{x})_{10} \neq n \vee(\vec{y})_{10} \neq m\right) \wedge e<C(\vec{x}, \vec{y}, a)\right) \vee d=1: & -\infty \\ \left((\vec{x})_{10}=n \wedge(\vec{y})_{10}=m\right) \wedge d=0: & e .\end{cases}
$$

A Equação 10.1 define três casos respectivamente: (i) O agente está em um estado não meta e há energia suficiente $\left(e \geq C(\vec{x}, \vec{y}, a)\right.$, portanto $e^{\prime}=e-C(\vec{x}, \vec{y}, a)$; (ii) $\mathrm{O}$ agente não está no estado meta, porém não há energia suficiente ou o agente está em um estado inválido $(d=1)$, portanto $e^{\prime}=-\infty$; (iii) $\mathrm{O}$ agente está no estado meta, portanto a energia permanece igual, i.e. $e^{\prime}=e$.

Foi utilizado $\theta_{u}=500$ nos experimentos.

\subsubsection{Domínio Mars Rover}

Neste domínio, o agente deve ir até $k$ locais alvos e tirar uma foto de cada local. Cada foto só pode ser tirada dentro de um intervalo de tempo e energia específicos.

Formalmente, as variáveis de estado são representadas por duas variáveis contínuas: tempo $T$ e energia $E$; e por $2 * k$ variáveis booleanas, em que $k$ variáveis representam a localização do robô $P_{i}$ e $k$ variáveis representam fotos obtidas pelo robô de cada localização $H_{i}$, tal que $1 \leq i \leq k$. O robô possui $2 * k+1$ ações, sendo que: (i) $k$ ações movimentam o robô para uma localização adjacente em relação a posição atual; (ii) $k$ ações tiram fotos de uma localização diferente; e (iii) 1 ação é ficar parado. Os estados meta são aqueles em que o robô obteve uma foto de cada localização $\left(H_{i}=1, \forall i\right)$ dentro do intervalo de tempo e energia especificados (por exemplo, $36000<t<50400$ e $e>1.62$ ).

Por exemplo, para o domínio com 1 local temos duas ações: tirar uma foto do local e ficar parado. O domínio foi testado com a seguinte função de custo que depende apenas das variáveis contínuas $H_{1}, T$ e $E$ :

$$
C\left(e, t, h_{1}\right)=\left\{\begin{array}{l}
e>1.62 \wedge t>36000 \wedge t<50400 \wedge h_{1}=0: 1.62 \\
h_{1}=1 \vee e<1.62 \vee t<36000 \vee t>50400: 0
\end{array}\right.
$$

As transições das variáveis $H_{1}, T$ e $\Gamma$ para a ação a de tirar uma foto do local são:

$$
\begin{gathered}
\operatorname{Pr}\left(h_{1}^{\prime}=1 \mid e, t, h_{1}, a\right)=\left\{\begin{array}{l}
(e>1.62 \wedge t>36000 \wedge t<50400) \vee h_{1}=1: \\
h_{1}=0 \vee e<1.62 \vee t<36000 \vee t>50400: \quad 0
\end{array}\right. \\
\operatorname{Pr}\left(t^{\prime} \mid e, t, h_{1}, a\right)=\delta\left(t^{\prime}-\left\{\begin{array}{l}
e>1.62 \wedge t>36000 \wedge t<50400 \wedge h_{1}=0: t+600 \\
h_{1}=1 \vee e<1.62 \vee t<36000 \vee t>50400: t
\end{array}\right)\right. \\
\operatorname{Pr}\left(e^{\prime} \mid e, t, h_{1}, a\right)=\delta\left(e^{\prime}-\left(e-C\left(e, t, h_{1}\right)\right)\right)
\end{gathered}
$$

Essas transições descrevem o seguinte cenário: A foto do local somente pode ser obtida no intervalo de tempo decorrido entre 36000 e 50400 e quando a energia é suficiente (i.e. maior que 1.62). Apenas uma foto pode ser obtida (Equação 10.3). O tempo total do agente é acrescido em 600 (Equação 10.4) e a energia decrescida em 1.62 quando uma foto é obtida (Equações 10.5 e 10.2).

Note que para essa instância não há a necessidade de utilizar a variável $p_{1}$, pois $p_{1}=1$ sempre. Duas representações foram elaboradas e testadas para esse domínio:

1. Como no caso do robô navegador, pode ser considerado que o orçamento definido pelo usuário 
está diretamente relacionado com a energia $E$ (i.e. $\Gamma=E$ ). Assim, a transição do orçamento e o custo seguem iguais à definida para a variável $E$ (Equações 10.5 e 10.2).

2. O orçamento não está diretamente relacionado com a energia e poderia incluir outros custos, assim é necessário criar uma nova variável $\Gamma$. Para essa configuração o domínio foi testado com a seguinte função de custo:

$$
C(e, t)= \begin{cases}e<10 \wedge t>36000: & 104.94 \\ e<10 \wedge t<36000: & 52.47 \\ e>10: & 30.40\end{cases}
$$

e a função de transição para a variável $\Gamma$ é definida por:

$$
\operatorname{Pr}\left(\theta^{\prime} \mid e, t, \theta, h_{1}, a\right)=\delta\left(\theta^{\prime}-(\theta-C(e, t))\right.
$$

O orçamento $\theta_{u}$ foi definido em 20 para a primeira configuração e em 1000 para a segunda.

\subsubsection{Análise do Tempo de Convergência}

A Figura 10.9 mostra os resultados obtidos no domínio do Robô Navegador usando a primeira opção de representação, i.e., a variável $\Gamma$ que mantém a quantidade de bateria restante do robô, é tratada como a única variável continua (apesar de apenas utilizar valores inteiros). É possível notar que em comparação com os demais algoritmos apresentados o algoritmo para tratamento de variáveis continuas possui o pior desempenho, algo já esperado devido ao tratamento da variável $\Gamma$ como contínua.

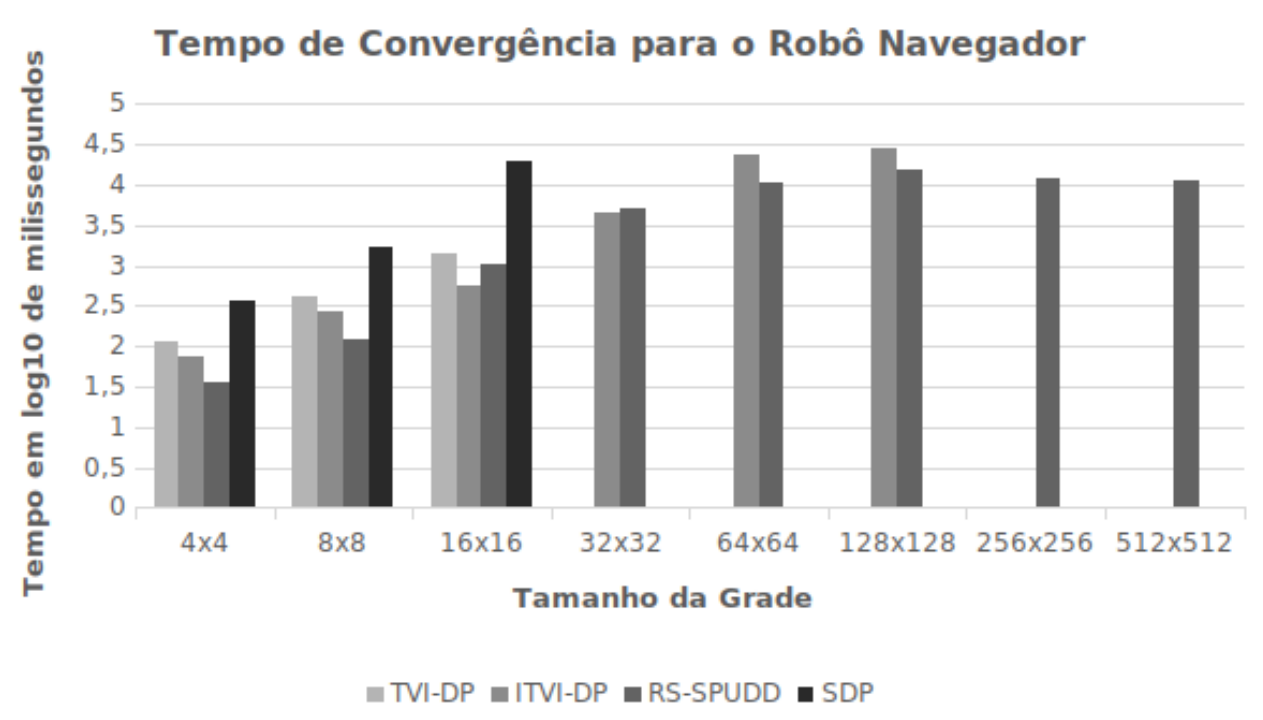

Figura 10.9: Tempo de convergência para o domínio do Robô Navegador.

Nas Figuras 10.10 e 10.11 são mostrados os resultados obtidos para o domínio Mars Rover para as duas configurações descritas, respectivamente. São mostrados os resultados variando $k$, isto é variando a quantidade de posições e fotos requeridas.

A Figura 10.10 mostra o resultado para a primeira configuração em que o orçamento definido pelo usuário está diretamente relacionado com a energia $E$ e a Figura 10.11 mostra o resultado em que o orçamento definido pelo usuário não está diretamente relacionado com a energia $E$.

Note que em ambas as configurações o aumento na complexidade do problema (aumento de posições e fotos requeridas) afeta consideravelmente o tempo de resposta do algoritmo para horizontes 


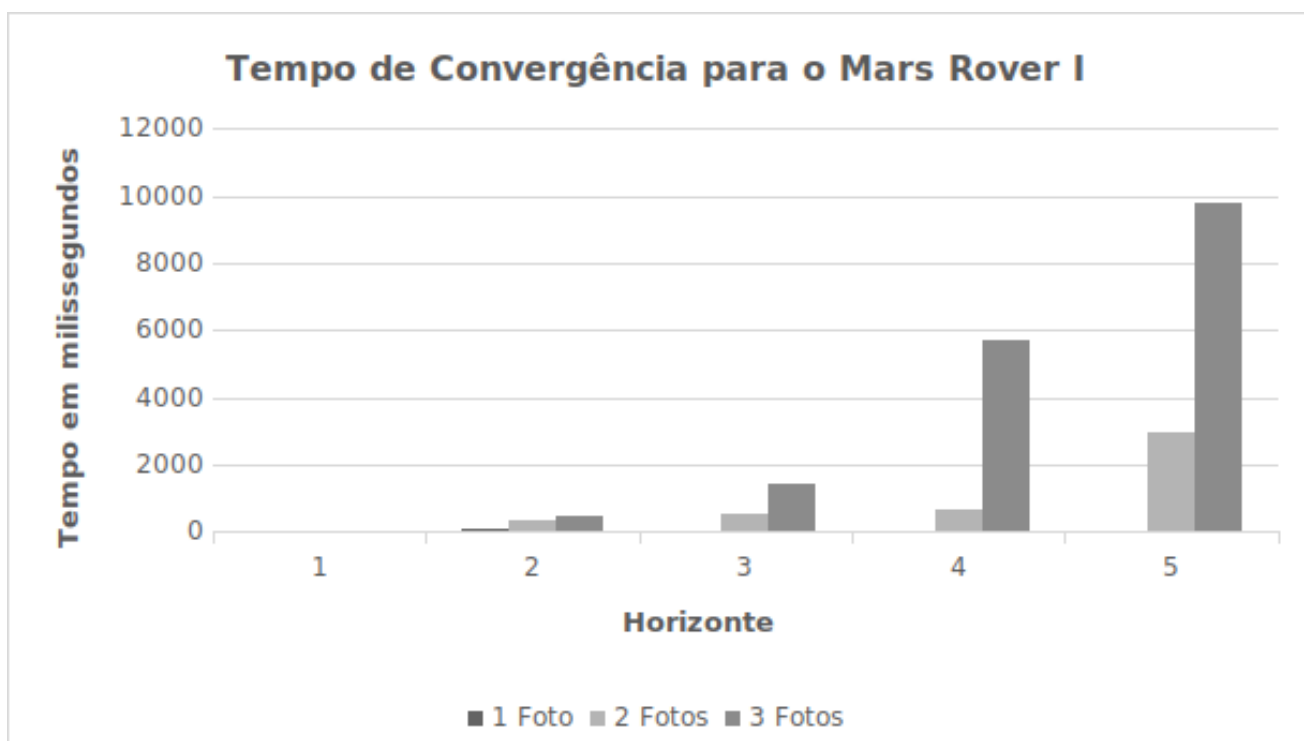

Figura 10.10: Tempo de convergência para a primeira configuração do domínio Mars Rover.

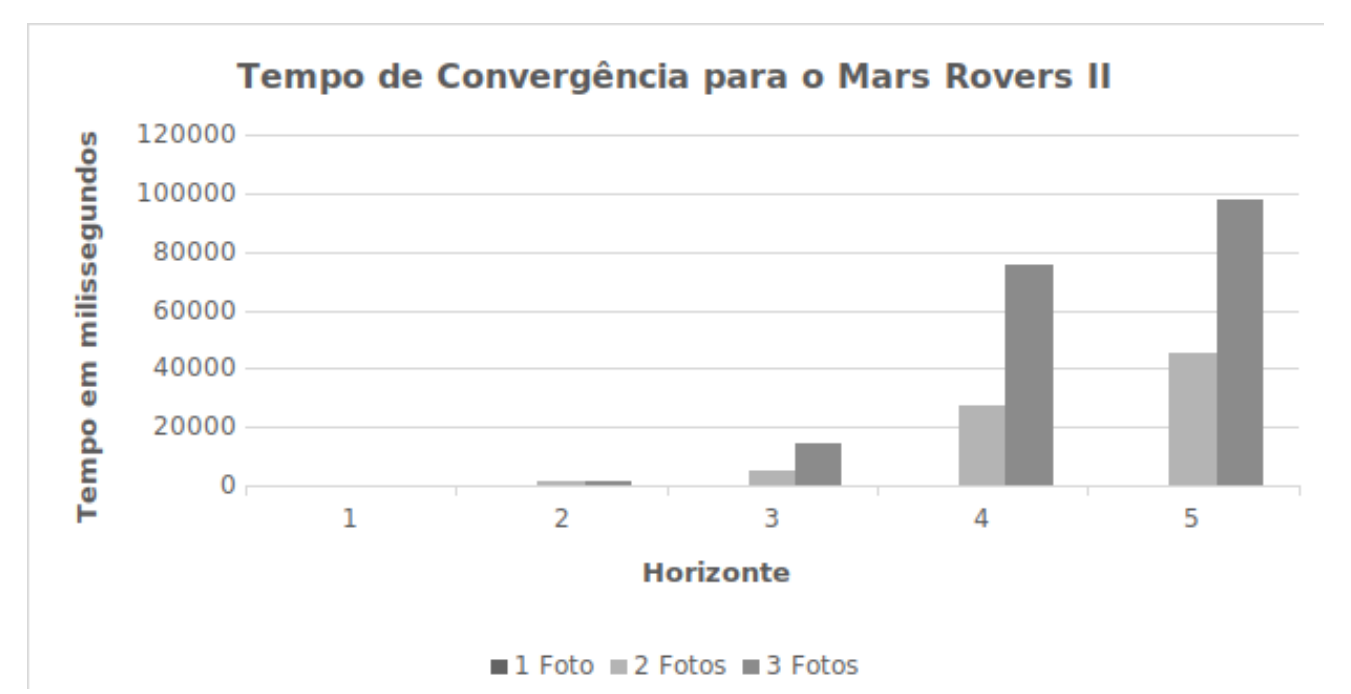

Figura 10.11: Tempo de convergência para a segunda configuração do domínio Mars Rover.

maiores do que 2. Além disso, note que a segunda configuração tem um tempo de execução 10 vezes maior que a primeira. 


\section{Capítulo 11}

\section{Considerações Finais e Trabalhos Futuros}

Neste trabalho RS-MDPs foram revisitados e um novo problema foi proposto com o objetivo de encontrar o orçamento mínimo $\theta_{\min }$ tal que a probabilidade de custo-limite $P^{*}\left(s, \theta_{\min }\right)$ é máxima para todos os estados $s$. Como resultado, foi demonstrado que dado um orçamento suficientemente grande, é possível garantir que a probabilidade de custo-limite ótima converge em $\theta$ quando $P^{*}(s, \theta)=P^{*}\left(s, \theta-c_{\max }\right)$. O algoritmo TVI-DP foi modificado para encontrar $\theta_{\min }$, tal algoritmo modificado é chamado de ITVI-DP. Foram também incluídas duas melhorias nesse algoritmo: (i) uma condição de parada adicional para quando o algoritmo atinge o ponto de convergência; e (ii) a geração de estados sobre demanda, reduzindo o número explícito de estados declarados.

Além disso, a versão fatorada de RS-MDPs foi desenvolvida e o algoritmo RS-SPUDD que explora as independências condicionais da função de transição foi proposto. Os algoritmos propostos (ITVIDP e RS-SPUDD) foram avaliados em termos de tempo de execução e consumo de memória para dois domínios (Robô Navegador e SysAdmin) do IPCC 2011. Os resultados dessa avaliação para os domínios testados indicam que o algoritmo RS-SPUDD possuí maior escalabilidade que os demais algoritmos, sendo capaz de resolver instâncias que os demais algoritmos não conseguiram, além de resolver com maior rapidez instâncias menores na maioria dos casos.

Por fim, a ampliação de RS-MDPs para a inclusão de variáveis contínuas foi abordada e experimentos foram realizados sobre dois domínios, Robô Navegador e Mars Rover. Os experimentos realizados para esses domínios mostraram que é possível resolver problemas mais complexos (i.e. com variáveis contínuas), porém o custo computacional aumenta consideravelmente.

Algumas das oportunidades de trabalhos futuros incluem:

- Soluções eficientes utilizando apenas o espaço de estados original: O maior desafio em garantir maior escalabilidade para as soluções propostas têm origem na utilização do espaço de estados aumentado. A utilização do espaço de estados aumentado gera um grande overhead com a explosão na quantidade de estados, assim soluções eficientes que utilizem somente o espaço de estados original são a chave para resolver instâncias ainda maiores.

- Extensão do problema e das soluções propostas para MDPs com função recompensa: Uma extensão natural do problema reside na extensão da função custo para uma função recompensa, permitindo assim um maior número de problemas modelados.

- Soluções baseadas em iteração de política e utilização de aproximadores para a política ótima: Outro grande desafio para maior escalabilidade reside na capacidade de prever como a política ótima evolui em função do orçamento.

- Extensão para otimização multiobjetivos: Outra extensão natural desse problema é a abordagem multiobjetivo em que desejaríamos garantir um mínimo de probabilidade de alcançar a meta além de limitar o orçamento máximo gasto. 
- Análise mais abrangente: Realizar uma análise mais minuciosa utilizando um número maior de domínios e variando todos parâmetros relevantes. 


\section{Referências Bibliográficas}

[Bel57] R. E. Bellman. Dynamic Programming. Princeton University Press, USA, 1957. 16

[Ber95] Dimitri P. Bertsekas. Dynamic Programming and Optimal Control, volume 1. Athena Scientific Belmont, MA, 1995. 16

[BFG $\left.{ }^{+} 93\right]$ Ruth Iris Bahar, Erica A. Frohm, Charles M. Gaona, Gary D. Hachtel, Enrico Macii, Abelardo Pardo e Fabio Somenzi. Algebraic decision diagrams and their applications. Em Proceedings of the International Conference on Computer-Aided Design (ICCAD), páginas 188-191, Los Alamitos, CA, USA, 1993. IEEE Computer Society Press. 5

[Bry86] Randal E. Bryant. Graph-based Algorithms for Boolean Function Manipulation. IEEE Transactions on Computers, 35(8):677-691, 1986. 5

[BT91] Dimitri P. Bertsekas e John N. Tsitsiklis. An analysis of stochastic shortest path problems. Math. Oper. Res., 16(3):580-595, 1991. 15

[BY10] Dimitri P. Bertekas e Huizhen Yu. Distributed asynchronous policy iteration in dynamic programming. Em 48th Annual Allerton Conference on Communication, Control, and Computing, páginas 1368-1375, 2010. 19

[DG93] Paul Dagum e Adam Galper. Forecasting sleep apnea with dynamic network models. Em Proceedings of the Ninth international conference on Uncertainty in artificial intelligence, páginas 64-71. Morgan Kaufmann Publishers Inc., 1993. 21

[DG07] Peng Dai e Judy Goldsmith. Topological value iteration algorithm for Markov decision processes. Em Manuela M. Veloso, editor, Proceedings of International Joint Conferences on Artificial Intelligence, páginas 1860-1865, 2007. 1, 2, 16, 19

[DSdB11] Karina Valdivia Delgado, Scott Sanner e Leliane Nunes de Barros. Efficient solutions to factored MDPs with imprecise transition Probabilities. Artificial Intelligence, 175(910):1498-1527, 2011. 25

[FD16] V. Freire e K. V. Delgado. Extreme risk averse policy for goal-directed risk-sensitive Markov decision process. Em 5th Brazilian Conference on Intelligent Systems (BRACIS), páginas 79-84, 2016. 25

[GB13] Hector Geffner e Blai Bonet. A Concise Introduction to Models and Methods for Automated Planning, volume 8. Morgan \& Claypool Publishers, 2013. 15

[GF15] Javier García e Fernando Fernández. A comprehensive survey on safe reinforcement learning. Journal of Machine Learning Research, 16:1437-1480, 2015. 25

[GW05] P. Geibel e F. Wysotzki. Risk-sensitive reinforcement learning applied to control under constraints. J. Artif. Intell. Res.(JAIR), 24:81-108, 2005. 25

[HM72] Ronald A. Howard e James E. Matheson. Risk-sensitive markov decision processes. Management Science, 18(7):356-369, 1972. 25 
[HSAHB99] Jesse Hoey, Robert St-Aubin, Alan Hu e Craig Boutilier. SPUDD: Stochastic planning using decision diagrams. Em Proceedings of the Fifteenth Conference on Uncertainty in Artificial Intelligence, páginas 279-288. Morgan Kaufmann, 1999. 2, 22, 61

[HYV14] P Hou, William Yeoh e Pradeep Varakantham. Revisiting Risk-Sensitive MDPs: New Algorithms and Results. Proceedings International Conference on Automated Planning and Scheduling, ICAPS, páginas 136-144, 2014. 1, 26, 27, 28, 29, 30, 31

[KKY06] Yoshinobu Kadota, Masami Kurano e Masami Yasuda. Discounted Markov decision processes with utility constraints. Computers and Mathematics with Applications, $51(2): 279-284,2006.25$

[Mar52] Harry Markowitz. Portfolio selection. In Journal of Finance, 7:77-91, 1952. 25

[NEG05] Arnab Nilim e Laurent El Ghaoui. Robust control of Markov decision processes with uncertain Transition Matrices. Operations Research, 53(5):780-798, 2005. 25

[Pat01] Stephen D Patek. On terminating Markov decision processes with a risk-averse objective function. Automatica, 37(9):1379-1386, 2001. 25

[Put94] Martin L. Puterman. Markov Decision Processes. Wiley Series in Probability and Mathematical Statistics. John Wiley and Sons, New York, 1994. 1

[SDdB11] Scott Sanner, Karina Valdivia Delgado e Leliane Nunes de Barros. Symbolic Dynamic Programming for Discrete and Continuous State MDPs. Em Proceedings of the TwentySeventh Conference on Uncertainty in Artificial Intelligence, UAI'11, páginas 643-652, 2011. 2,37

[SKK01] Makoto Sato, Hajime Kimura e Shibenobu Kobayashi. Td algorithm for the variance of return and mean-variance reinforcement learning. Transactions of the Japanese Society for Artificial Intelligence, 16(3):353-362, 2001. 25

[SM06] S. Sanner e S. McIlraith. An ordered theory resolution calculus for hybrid reasoning in first-order extensions of description logic. Em Proceedings of the 10th International Conference on Principles of Knowledge Representation and Reasoning (KR-06), páginas 100-111, 2006. 2

[Tar71] R. Tarjan. Depth-first search and linear graph algorithms. Em 12th Annual Symposium on Switching and Automata Theory (swat 1971), páginas 114-121, Oct 1971. 18, 43

[YLY98] Stella X Yu, Yuanlie Lin e Pingfan Yan. Optimization models for the first arrival target distribution function in discrete time. Journal of Mathematical Analysis and Applications, 225(1):193 - 223, 1998. 1, 25, 26, 31 